\title{
Scanning X-Ray Nanodiffraction on Dictyostelium discoideum
}

\author{
DiSSERTATION \\ zur Erlangung des mathematisch-naturwissenschaftlichen Doktorgrades \\ Doctor rerum naturalium \\ der Georg-August-Universität Göttingen \\ im Promotionsprogramm PROPHYS \\ der Georg-August University School of Science (GAUSS) \\ vorgelegt von \\ Marius Patrick Priebe \\ aus Goslar
}

Göttingen, 2015 


\section{Betreuungsausschuss}

Prof. Dr. Tim Salditt

Institut für Röntgenphysik

Georg-August-Universität Göttingen

Prof. Dr. Jörg Enderlein

III. Physikalisches Institut

Georg-August-Universität Göttingen

\section{Mitglieder der Prüfungskommission}

Referent: $\quad$ Prof. Dr. Tim Salditt

Institut für Röntgenphysik

Georg-August-Universität Göttingen

1. Korreferent: Prof. Dr. Jörg Enderlein

III. Physikalisches Institut

Georg-August-Universität Göttingen

Weitere Mitglieder der Prüfungskommission

Prof. Dr. Eberhard Bodenschatz

Max-Planck-Institut für Dynamik und Selbstorganisation

Georg-August-Universität Göttingen

Prof. Dr. Sarah Köster

Institut für Röntgenphysik

Georg-August-Universität Göttingen

Prof. Dr. Simone Techert

Institut für Röntgenphysik

Georg-August-Universität Göttingen

Dr. Jochen Hub

Institut für Mikrobiologie und Genetik

Georg-August-Universität Göttingen

Tag der mündlichen Prüfung: 4.2.2015 


\section{Contents}

List of figures $\quad$ IV

Bibliograpy

1. Introduction 1

2. Fundamentals 3

2.1. Dictyostelium discoideum . . . . . . . . . . . . . . . 3

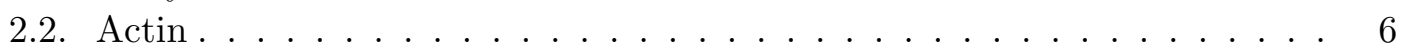

2.2.1. Actin Structure . . . . . . . . . . . . . . . . . 6

2.2.2. Networks . . . . . . . . . . . . . . . . 7

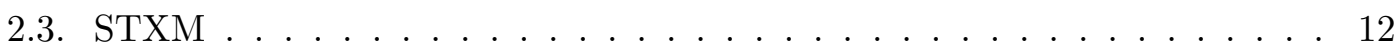

$\begin{array}{lr}\text { 3. Materials and Methods } & \mathbf{1 5}\end{array}$

3.1. Cell Lines . . . . . . . . . . . . . . . . . . . . . . . . . 15

3.2. Cell Culture . . . . . . . . . . . . . . . . . . . . 16

3.2.1. Buffers and Media . . . . . . . . . . . . . . . 16

3.2.2. Cell Growth . . . . . . . . . . . . . . . . . . . . 17

3.2.3. Active Preparation of Chemotactically Competent Cells . . . . . . 17

3.2.4. Passive Preparation of Chemotactically Competent Cells . . . . . . 18

3.3. Sample Fixation . . . . . . . . . . . . . . . . . . . 18

3.3.1. Frozen Hydrated Cells . . . . . . . . . . . . . . . . 18

3.3.2. Freeze Dried Cells . . . . . . . . . . . . . . . . . . 20

3.3.3. Chemically Fixed Cells . . . . . . . . . . . . . . . . . 20

3.3.4. Living Cells . . . . . . . . . . . . . . . . 21

3.4. Sample Environments . . . . . . . . . . . . . . . . . 22

3.4.1. Cryostream . . . . . . . . . . . . . . . 22

3.4.2. Sample Mounting Procedure . . . . . . . . . . . . . . . 23

3.4.3. Microfluidic Chambers for Living Cells: . . . . . . . . . . . . . . . 24

3.5. X-ray Fluorescence . . . . . . . . . . . . . . . . . . . . . 25

3.6. X-ray Setups . . . . . . . . . . . . . . . . 26

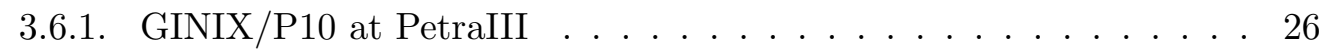

3.6.2. ID13 at ESRF . . . . . . . . . . . . . . 27

3.6.3. cSAXS at SLS . . . . . . . . . . . . . . . 28

4. Streak Finder $\quad 32$

4.1. Algorithm . . . . . . . . . . . . . . . . . . . 32 
4.2. Normalization . . . . . . . . . . . . . . . . . . . . . 36

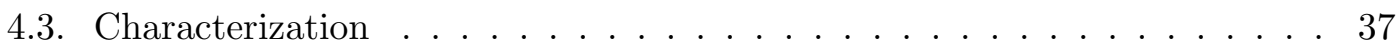

4.4. Possible Future Modifications . . . . . . . . . . . . . . . . . . . . 42

4.5. Different Definitions . . . . . . . . . . . . . . . . . . 43

5. Results $4 \mathbf{4 6}$

5.1. Reciprocal Space . . . . . . . . . . . . . . . . . . . . . 46

5.2. Real Space . . . . . . . . . . . . . . . . . . . . . . . . . 49

5.3. Results from ID13 . . . . . . . . . . . . . . . . . . . 56

5.4. Ptychographic Phase Imaging . . . . . . . . . . . . . . . . 58

5.5. Fluorescence Measurements . . . . . . . . . . . . . . . . . . . 60

6. Conclusion and Outlook 66

$\begin{array}{ll}\text { 7. Danksagung } & 69\end{array}$

$\begin{array}{ll}\text { A. Additional Material } & \mathbf{7 1}\end{array}$

A.1. Fluorescence Data . . . . . . . . . . . . . . . . . . . . . . 71

$\begin{array}{ll}\text { B. Reciprocal Space Transformation } & 75\end{array}$

$\begin{array}{ll}\text { C. Matlab Scripts } & \mathbf{7 7}\end{array}$

C.0.1. Streak Finder Algorithm . . . . . . . . . . . . . . . . . . . 77

C.0.2. Fluorescence Mapping . . . . . . . . . . . . . . . . . . . . . 81

$\begin{array}{ll}\text { D. Manuals } & \mathbf{8 9}\end{array}$

D.1. Cryo Wiki . . . . . . . . . . . . . . . . . . . . . . . 89

D.1.1. Cryostream . . . . . . . . . . . . . . . . . . 89

D.1.2. Automatic Dewar Refill System . . . . . . . . . . . . . . . 91

D.2. Fluorescence Detector . . . . . . . . . . . . . . . 93

D.2.1. Data . . . . . . . . . . . . . . . . . . . . 93

D.2.2. Setup . . . . . . . . . . . . . . . 93

D.2.3. Connect... . . . . . . . . . . . . . . . . . 93

D.2.4. Energy Calibration . . . . . . . . . . . . . . . . . . . 94

D.2.5. Spec Commands . . . . . . . . . . . . . . . . . . . . 94

D.2.6. Implementation into Spec . . . . . . . . . . . . . . . . . 94

D.2.7. Use Spec Counting Time for Mca . . . . . . . . . . . . . . . . 95 


\section{List of Figures}

2.1. Different representations of G- and F-actin molecules based on visualisations from Protein data bank datasets. . . . . . . . . . . . . . . 8

2.2. Image and sketch of $D$. discoideum cell. . . . . . . . . . . . . . . . 10

2.3. Illustration of scanning nano-SAXS, collage of a corresponding typical dataset for overview and generated contrast maps of same dataset. . . . . 13

3.1. Figure on preparation steps: Illustrations of the cryoplunging process, the cryogenic mounting procedure, sketch and image of microfluidic chamber for x-ray experiments by ibidi and corresponding micrographs of cryogenic sample after plunging, as seen by inline microscope and darkfield map. . . 19

3.2. Illustration of masks used for SAXS contrast determinations. . . . . . . . 30

3.3. Sketches of the x-ray diffractometers at P10, cSAXS and ID13. . . . . . . 31

4.1. Illustration of Streak Finder algorithm on a real diffraction pattern. . . . 35

4.2. Definition of nomenclature for detector Rois. . . . . . . . . . . . . . 38

4.3. Results of Streak Finder from simulated data: Influence of insensitive detector areas. . . . . . . . . . . . . . . . . 39

4.4. Results of Streak Finder from simulated data: Influence of streak width. . 40

4.5. Results of Streak Finder from simulated data: Influence of background levels and radial streak intensity decay. . . . . . . . . . . . . . . . 41

4.6. Results of Streak Finder from simulated data: Box shaped streaks. . . . . 41

4.7. Comparison of different anisotropy parameter definitions. . . . . . . . . . 45

5.1. Determination of average scattering signal from a cell and the background. 50

5.2. Intenisty curves $I\left(q_{r}\right)$ as functions of the radial scattering vector $q_{r}$ for single streaks. . . . . . . . . . . . . . . . 51

5.3. Simulation of far-field intensity distribution for small fibre bundles: Sketch of fibre bundle geometry and resulting intensity distributions for different orietations of fibre bundles. . . . . . . . . . . . . . . . 52

5.4. Stxm contrast maps from scanning SAXS datasets of frozen-hydrated $D$. discoideum cells. . . . . . . . . . . . . . . . . . 54

5.5. Experiments on initially living cells and comparison of preparation protocols. 55

5.6. Comparison of STXM contrast maps and fluorescence micrograph (F-actin and Myosin-II label) of D. discoideum cells. . . . . . . . . . . . . . 56

5.7. Diffraction dataset of freeze-dried D. discoideum obtained at ID13. . . . . 58

5.8. STXM maps of whole D. discoideum cell with obtained at ID13. . . . . . 59 
5.9. Ptychographic reconstructions of several $D$. discoideum and D. radiodurans cells in frozen-hydrated as well as freeze-dried condition. . . . . . . . . 61

5.10. Results from fluorescence acquisition, example data curves, and element map of one scan. . . . . . . . . . . . . . . . . . . . 63

5.11. Elemental maps from raster scans on $D$. discoideum of four energy ranges for four different scanning regions. . . . . . . . . . . . . . . . 64

A.1. Development of fluorescence spectra over several days of experiments at the GINIX instrument, P10. . . . . . . . . . . . . . . . 71

A.2. Development of a sample of $D$. discoideum on a silicon nitride membrane during the preparation of frozen-hydrated sample. . . . . . . . . . . 72

A.3. Comparison of three samples of $D$. discoideum on silicon nitride membranes before the first and before the last step of the preparation protocol for frozen-hydrated samples. . . . . . . . . . . . . . . . . 73

A.4. X-ray transmission curve of $1 \mathrm{~cm}$ air for $E_{P h} \in 10 \mathrm{eV} \ldots 2000 \mathrm{eV}$. . . . . 74 


\section{Bibliography}

[1] J. S. King and R. H. Insall: Chemotaxis: finding the way forward with Dictyostelium. Trends in Cell Biology, 19(10):523 - 530, 2009.

[2] P. Friedl, S. Borgmann and E.-B. Bröcker: Amoeboid leukocyte crawling through extracellular matrix: lessons from the Dictyostelium paradigm of cell movement. J. Leukocyte Biol., 70(4):491-509, 2001.

[3] S. J. Annesley and P. R. Fisher: Dictyostelium discoideum-a model for many reasons. Mol. Cell. Biochem., 329:73-91, 2009.

[4] J. Linkner, G. Witte, H. Zhao, A. Junemann, B. Nordholz, P. RungeWollmann, P. LAppalainen and J. Faix: The inverse BAR domain protein IBARa drives membrane remodeling to control osmoregulation, phagocytosis and cytokinesis. J. Cell Sci., 127(6):1279-1292, 2014.

[5] H. M. Berman, J. Westbrook, Z. Feng, G. Gilliland, T. N. Bhat, H. WeisSig, I. N. Shindyalov and P. E. Bourne: The Protein Data Bank. Nucleic Acids Res., 28(1):235-242, 2000.

[6] P. J. M. Van Haastert: Chemotaxis: insights from the extending pseudopod. $J$. Cell Sci., 123:3031-3037, 2010.

[7] S. A. Koestler, A. Steffen, M. Nemethova, M. Winterhoff, N. Luo, J. M. Holleboom, J. Krupp, S. Jacob, M. Vinzenz, F. Schur, K. Schlüter, P. W. Gunning, C. Winkler, C. Schmeiser, J. Faix, T. E. B. Stradal, J. V. Small and K. RotTneR: Arp2/3 complex is essential for actin network treadmilling as well as for targeting of capping protein and cofilin. Mol. Biol. Cell, 24(18):2861-2875, 2013.

[8] C. Blum. PhD thesis, Universität Göttingen, In preparation.

[9] M. M. A. E. Claessens, C. Semmrich, L. Ramos and A. R. Bausch: Helical twist controls the thickness of F-actin bundles. PNAS, 105(26):8819-8822, July 2008.

[10] B. Weinhausen, J.-F. Nolting, C. Olendrowitz, J. Langfahl-Klabes, M. Reynolds, T. SAlditT and S. KÖsteR: X-ray nano-diffraction on cytoskeletal networks. New J. Phys., 14(8):085013, 2012.

[11] B. Weinhausen: Scanning X-ray nano-diffraction on eukaryotic cells: from freezedried to living cells. PhD thesis, Georg-August Universität Göttingen, 2013. 
[12] K. GieweKemeyer: A study on new approaches in coherent x-ray microscopy of biological specimens. PhD thesis, Universität Göttingen, 2011.

[13] K. Giewekemeyer, S. P. Krüger, S. Kalbfleisch, M. Bartels, C. Beta and T. SALDITT: X-ray propagation microscopy of biological cells using waveguides as a quasipoint source. Phys. Rev. A, 83(2):023804, Feb 2011.

[14] R. N. Wilke: Coherent X-Ray Diffractive Imaging on the Single-Cell-Level of Microbial Samples: Ptychography, Tomography, Nano-Diffraction and WaveguideImaging. PhD thesis, Georg-August University School of Science (GAUSS) Göttingen, 2014.

[15] E. Lima, A. Diaz, M. Guizar-Sicairos, S. Gorelick, P. Pernot, T. Schleier and A. Menzel: Cryo-scanning x-ray diffraction microscopy of frozen-hydrated yeast. Journal of Microscopy, 249(1):1-7, Jan. 2013.

[16] M. Priebe, M. Bernhardt, C. Blum, M. Tarantola, E. Bodenschatz and T. SALDITT: Scanning X-Ray Nanodiffraction on Dictyostelium discoideum. Biophys. J., 107(11):2662-2673, December 2014.

[17] L. Eichinger, J. A. Pachebat, G. Glockner, M.-A. Rajandream, R. Sucgang, M. Berriman, J. Song, R. Olsen, K. Szafranski, Q. Xu, B. Tunggal, S. Kummerfeld, M. Madera, B. A. Konfortov, F. Rivero, A. T. Bankier, R. Lehmann, N. Hamlin, R. Davies, P. Gaudet, P. Fey, K. Pilcher, G. Chen, D. Saunders, E. Sodergren, P. Davis, A. Kerhornou, X. Nie, N. Hall, C. Anjard, L. Hemphill, N. Bason, P. Farbrother, B. Desany, E. Just, T. Morio, R. Rost, C. Churcher, J. Cooper, S. Haydock, N. van DriessChe, A. Cronin, I. Goodhead, D. Muzny, T. Mourier, A. Pain, M. Lu, D. Harper, R. Lindsay, H. Hauser, K. James, M. Quiles, M. Madan Babu, T. Saito, C. Buchrieser, A. Wardroper, M. Felder, M. Thangavelu, D. Johnson, A. Knights, H. Loulseged, K. Mungall, K. Oliver, C. Price, M. A. Quail, H. Urushihara, J. Hernandez, E. Rabbinowitsch, D. Steffen, M. Sanders, J. Ma, Y. Kohara, S. Sharp, M. Simmonds, S. Spiegler, A. Tivey, S. Sugano, B. White, D. Walker, J. Woodward, T. Winckler, Y. Tanaka, G. Shaulsky, M. Schleicher, G. Weinstock, A. Rosenthal, E. C. Cox, R. L. Chisholm, R. Gibbs, W. F. Loomis, M. Platzer, R. R. Kay, J. Williams, P. H. Dear, A. A. Noegel, B. Barrell and A. Kuspa: The genome of the social amoeba Dictyostelium discoideum. Nature, 435(7038):43-57, May 2005.

[18] R. H. Kessin: Dictyostelium - Evolution, Cell Biology, and the Development of Multicellularity. Cambridge University Press, 2001.

[19] R. L. Chisholm and R. A. Firtel: INSIGHTS INTO MORPHOGENESIS FROM A SIMPLE DEVELOPMENTAL SYSTEM. Nat Rev Mol Cell Biol, 5(7):531-541, July 2004. 
[20] R. Arya, A. Bhattacharya and K. S. Saini: Dictyostelium discoideum - a promising expression system for the production of eukaryotic proteins. The FASEB Journal, 22(12):4055-4066, 2008.

[21] C. Blum, M. Tarantola and E. Bodenschatz: Curvotaxis. in preparation, 2015.

[22] K. F. Swaney, C.-H. Huang and P. N. Devreotes: Eukaryotic Chemotaxis: A Network of Signaling Pathways Controls Motility, Directional Sensing, and Polarity. Annu. Rev. Biophys., 39(1):265-289, Apr. 2010.

[23] P. J. Van Hanstert: A Stochastic Model for Chemotaxis Based on the Ordered Extension of Pseudopods. Biophys. J., 99(10):3345-3354, 2010.

[24] P. J. M. Van HaAstert: Amoeboid Cells Use Protrusions for Walking, Gliding and Swimming. PLoS One, 6(11):e27532, 112011.

[25] L. Bosgraaf and P. J. M. Van HaAstert: Navigation of Chemotactic Cells by Parallel Signaling to Pseudopod Persistence and Orientation. PLoS One, 4(8):e6842, 082009.

[26] B. Alberts, A. Johnson, J. Lewis, M. Raff, K. Roberts and P. Walter: Molecular biology of the cell. Garland Science, New York, 5th ed., 2008.

[27] L. Blanchoin, R. Boujemaa-Paterski, C. Sykes and J. Plastino: Actin Dynamics,Architecture, and Mechanics in Cell Motility. Physiol. Rev., 94:235-263, 2014.

[28] G. Koenl and J. G. McNally: Myosin II redistribution during rear retraction and the role of filament assembly and disassembly. Cell Biol. Int., 26(3):287-296, 2002 .

[29] G. Gerisch: Periodische Signale steuern die Musterbildung in Zellverbänden. Naturwissenschaften, 58(9):430-438, 1971.

[30] F. Siegert und C. J. Weijer: Spiral and concentric waves organize multicellular Dictyostelium mounds. Current Biology, 5(8):937-943, 1995.

[31] P. Foerster, S. Muller und B. Hess: Curvature and spiral geometry in aggregation patterns of Dictyostelium discoideum. Development, 109(1):11-16, 1990.

[32] C. Westendorf: Oscillatory Dynamics of the Actin Cytoskeleton. Doktorarbeit, Georg-August-Universität Göttingen, 2012.

[33] F. Knoch, M. Tarantola, E. Bodenschatz und W.-J. Rappel: Modeling self-organized spatio-temporal patterns of PIP 3 and PTEN during spontaneous cell polarization. Phys. Biol., 11(4):046002, 2014. 
[34] G. Gerisch, B. Schroth-Diez, A. Müller-Taubenberger und M. Ecke: \{PIP3\} Waves and $\{$ PTEN\} Dynamics in the Emergence of Cell Polarity. Biophys $J, 103(6): 1170-1178,2012$.

[35] O. Medalia, M. Beck, M. Ecke, I. Weber, R. Neujahr, W. Baumeister und G. Gerisch: Organization of Actin Networks in Intact Filopodia. Curr. Biol., 17(1):79-84, Jan. 2007.

[36] S. A. Koestler, S. Auinger, M. Vinzenz, K. Rottner und J. V. Small: Differentially oriented populations of actin filaments generated in lamellipodia collaborate in pushing and pausing at the cell front. Nat. Cell Biol., 10(3):306-313, März 2008.

[37] M. TÖPPERWIEN: Röntgenstrukturanalyse von Aktinsuspensionen. Diplomarbeit, Georg-August-Universität Göttingen, 2012.

[38] O. Lieleg, M. M. A. E. Claessens, and A. R. Bausch: Structure and dynamics of cross-linked actin networks. Soft Matter, 10:218 - 225, 2010.

[39] S. Diez, G. Gerisch, K. Anderson, A. Müller-Taubenberger, and T. BRETSCHNEIDER: Subsecond reorganization of the actin network in cell motility and chemotaxis. PNAS, 102(21):7601-7606, 2005.

[40] O. Schulz, C. Pieper, M. Clever, J. Pfaff, A. Ruhlandt, R. H. Kehlenbach, F. S. Wouters, J. Grosshans, G. Bunt, and J. Enderlein: Resolution doubling in fluorescence microscopy with confocal spinning-disk image scanning microscopy. PNAS, 110(52):21000-21005, 2013.

[41] M. Vinzenz, M. Nemethova, F. Schur, J. Mueller, A. Narita, E. Urban, C. Winkler, C. Schmeiser, S. A. Koestler, K. Rottner, G. P. Resch, Y. MaedA, and J. V. SMALL: Actin branching in the initiation and maintenance of lamellipodia. J. Cell Sci., 125(11):2775-2785, 2012.

[42] D. F. Lusche, D. Wessels, and D. R. Soll: The Effects of Extracellular Calcium on Motility, pseudopod and Uropod Formation, Chemotaxis, and the Cortical Localization of Myosin ii in Dictyostelium discoideum. Cell Motil. Cytoskeleton, 66:567-587, 2009.

[43] J.-F. Rounyrenc and F. Travers: The First Step in the Polymerisation of Actin. European Journal of Biochemistry, 116:73-77, 1981.

[44] T. D. Pollard and J. A. Cooper: Actin, a central player in cell shape and movement. Science, 326(5957):1208-1212, Nov. 2009.

[45] E. Behrmann, M. Müller, P. Penczek, H. Mannherz, D. Manstein, and S. Raunser: Structure of the Rigor Actin-Tropomyosin-Myosin Complex. Cell, 150(2):327-338, July 2012. 
[46] E. F. Pettersen, T. D. Goddard, C. C. Huang, G. S. Couch, D. M. GreenBlatt, E. C. Meng, and T. E. FERRIN: Ucsf chimera-a visualization system for exploratory research and analysis. J. Comput. Chem., 25(13):1605-1612, 2004.

[47] T. D. Pollard: Rate constants for the reactions of atp-and adp-actin with the ends of actin filaments.. JCB, 103(6):2747-2754, 1986.

[48] K. C. Holmes, D. Popp, W. Gebhard, and W. Kabsch: Atomic model of the actin filament. Nature, 347(6288):44-49, Sept. 1990.

[49] M. Y. Ali, S. Uemura, K. Adachi, H. Itoh, K. Kinosita, and S. Ishiwata: Myosin $v$ is a left-handed spiral motor on the right-handed actin helix. Nat Struct Mol Biol, 9(6):464-467, June 2002.

[50] E. H. Egelman, N. Francis, and D. J. DeRosier: F-actin is a helix with a random variable twist. Nature, 298(5870):131-135, July 1982.

[51] J. van Mameren, K. Vermeulen, F. Gittes, and C. Schmidt: Leveraging single protein polymers to measure flexural rigidity. J. Phys. Chem. B, 113:3837-3844, 2009.

[52] D. S. Fudge, K. H. Gardner, T. Forsyth, C. Riekel, and J. M. Gosline: The Mechanical Properties of Hydrated Intermediate Filaments: Insights from Hagfish Slime Threads. Biophys J, 85(3):2015-2027, 2003.

[53] SchröDInger, LLC.: The pymol molecular graphics system, version 1.7.1.3.

[54] C. G. Dos Remedios, D. Chhabra, M. Kekic, I. V. Dedova, M. Tsubakihara, D. A. Berry, and N. J. Nosworthy: Actin Binding Protein: Regulation of Cytoskeletal Microfilaments. Physiol. Rev., 83(2):433-473, April 2003.

[55] R. Insall, A. Müller-Taubenberger, L. Machesky, J. Köhler, E. Simmeth, S. J. Atkinson, I. Weber, and G. Gerisch: Dynamics of the dictyostelium arp2/3 complex in endocytosis, cytokinesis, and chemotaxis. Cell Motil. Cytoskeleton, 50(3):115-128, 2001.

[56] C. Westendorf, A. Bae, C. Erlenkamper, E. Galland, C. Franck, E. BoDENSCHATZ, and C. BETA: Live cell flattening - traditional and novel approaches. PMC Biophysics, 3(1):9, 2010.

[57] T. Bretschneider, J. Jonkman, J. Köhler, O. Medalia, K. Barisic, I. WeBer, E. H. Stelzer, W. Baumeister, and G. Gerisch: Dynamic organization of the actin system in the motile cells of Dictyostelium. J. Muscle Res. Cell Motil., 23(7-8):639 - 649, 2002.

[58] J. Als-Nielsen and D. McMorrow: Elements of Modern X-ray Physics. 2001.

[59] D. M. Paganin: Coherent X-Ray Optics. New York: Oxford University Press, 2006. 
[60] W. LAUTERBORN and T. KURZ: Coherent optics : fundamentals and applications ; with 73 problems and complete solutions. Springer, 2003.

[61] N. Schneider, I. Weber, J. Faix, J. Prassler, A. Müller-Taubenberger, J. Köhler, E. Burghardt, G. Gerisch, and G. Marriott: A Lim Protein Involved in the Progression of Cytokinesis and Regulation of the Mitotic Spindle. Cell Motil. Cytoskeleton, 56:130-139, 2003.

[62] R. Sussman and M. Sussman: Cultivation of Dictyostelium discoideum in axenic medium. Biochem. Biophys. Res. Commun., 29(1):53-55, Oct. 1967.

[63] P. Fey, A. S. Kowal, P. Gaudet, K. E. Pilcher, and R. L. Chisholm: Protocols for growth and development of Dictyostelium discoideum. Nat. Protoc., 2(6):1307-1316, June 2007.

[64] R. Chisholm, S. Hopkinson, and H. Lodish: Superinduction of the Dictyostelium discoedium cell surface cAMP receptor by pulses of cAMP. PNAS, 84:1030-1034, 1987.

[65] G. P. Resch, E. Urban, and S. Jacob: The Actin Cytoskeleton in Whole Mount Preparations and Sections. In T. Mueller-REICHERT (ed.): Electron Microscopy of Model Systems, vol. 96 of Methods in Cell Biology, chap. 22, pp. 529-564. Elsevier Inc., 1st ed., 2010.

[66] G. P. Resch, M. Brandstetter, L. Königsmaier, E. Urban, and A. M. PICKL-HeRK: Immersion Freezing of Suspended Particles and Cells for CryoElectron Microscopy. CSHP, 2011(7):pdb.prot5642, 2011.

[67] G. P. Resch, M. Brandstetter, V. I. Wonesch, and E. Urban: Immersion Freezing of Cell Monolayers for Cryo-Electron Tomography. CSHP, 2011(7):pdb.prot5643, 2011.

[68] G. P. Resch, M. Brandstetter, A. M. Pickl-Herk, L. Königsmaier, V. I. Wonesch, and E. URBAn: Immersion Freezing of Biological Specimens: Rationale, Principles, and Instrumentation. CSHP, 2011(7):pdb.top118, 2011.

[69] B. Hof, J. Westerweel, T. M. Schneider, and B. Eckhardt: Finite lifetime of turbulence in shear flows. Nature, 443(7107):59-62, Sept. 2006.

[70] T. Gorniak: Melanosomen im Real- und Fourierraum. PhD thesis, Universität Heidelberg, 2013.

[71] V. Solé, E. Papillon, M. Cotte, P. Walter, and J. Susini: A multiplatform code for the analysis of energy-dispersive $X$-ray fluorescence spectra. Spectrochimica Acta Part B: Atomic Spectroscopy, 62(1):63-68, Jan. 2007.

[72] S. Kalbfleisch, H. Neubauer, S. P. Krüger, M. Bartels, M. Osterhoff, D. D. Mai, K. Giewekemeyer, B. Hartmann, M. Sprung, and T. Salditt: 
The Göttingen Holography Endstation of Beamline P10 at PETRA III/DESY. AIPConf.Proc., 1365(1):96-99, 2011.

[73] T. Salditt, S. Kalbfleisch, M. Osterhoff, S. P. Krüger, M. Bartels, K. Giewekemeyer, H. Neubauer, and M. Sprung: Partially coherent nanofocused $x$-ray radiation characterized by Talbot interferometry. Opt. Express, 19(10):9656-9675, May 2011.

[74] S. Kalbfleisch: A Dedicated Endstation for Waveguide-based X-Ray Imaging. $\mathrm{PhD}$ thesis, Universität Göttingen, 2012.

[75] Y. Takahashi, A. Suzuki, N. Zettsu, Y. Kohmura, Y. Senba, H. Ohashi, K. YAMAUCHI, and T. IshIKAWA: Towards high-resolution ptychographic x-ray diffraction microscopy. Phys. Rev. B, 83(21):214109, June 2011.

[76] R. N. Wilke, M. Vassholz, and T. SalditT: Semi-transparent central stop in high-resolution x-ray ptychography using kirkpatrick-baez focusing. Acta Crystallogr.A, 69(5):490-497, 2013.

[77] B. Lengeler, C. G. Schroer, M. Kuhlmann, B. Benner, T. F. Günzler, O. Kurapova, F. Zontone, A. Snigirev, and I. Snigireva: Refractive x-ray lenses. Journal of Physics D: Applied Physics, 38(10A):A218, 2005.

[78] B. Lengeler, C. G. Schroer, B. Benner, A. Gerhardus, T. F. Günzler, M. Kuhlmann, J. Meyer, and C. Zimprich: Parabolic refractive X-ray lenses. J. Synchrotron Rad., 9(3):119-124, May 2002.

[79] C. Ponchut, J. M. Rigal, J. Clément, E. Papillon, A. Homs, and S. PeTITDEMANGE: MAXIPIX, a fast readout photon-counting X-ray area detector for synchrotron applications. Journal of Instrumentation, 6(01):C01069, 2011.

[80] R. N. Wilke, M. Priebe, M. Bartels, K. Giewekemeyer, A. Diaz, P. KarviNEN, and T. SALDITT: Hard X-ray imaging of bacterial cells: nano-diffraction and ptychographic reconstruction. Opt. Express, 20(17):19232-19254, Aug. 2012.

[81] O. Bunk, M. Bech, T. H. Jensen, R. Feidenhans'L, T. Binderup, A. Menzel, and F. PfeIfFer: Multimodal x-ray scatter imaging. New Journal of Physics, 11(12):123016, 2009.

[82] B. Weinhausen, O. Saldanha, R. N. Wilke, C. Dammann, M. Priebe, M. Burghammer, M. Sprung, and S. Köster: Scanning x-ray nanodiffraction on living eukaryotic cells in microfluidic environments. Phys. Rev. Lett., 112(8):088102, Feb. 2014.

[83] J.-W. Chu and G.-A. Vотн: Coarse-Grained Modeling of the Actin Filament Derived from Atomistic-Scale Simulations. Biophys. J., 90:1572-1572, 2006. 
[84] K. Giewekemeyer, P. Thibault, S. Kalbfleisch, A. Beerlink, C. M. Kewish, M. Dierolf, F. Pfeiffer, and T. SAlditt: Quantitative biological imaging by ptychographic x-ray diffraction microscopy. PNAS, 107(2):529-534, 2010.

[85] T. Paunesku, S. Vogt, J. Maser, B. Lai, and G. Woloschak: X-ray fluorescence microprobe imaging in biology and medicine. J. Cell. Biochem., 99(6):14891502, 2006.

[86] K. Giewekemeyer, M. Hantke, C. Beta, R. Tucoulou, and T. Salditt: Fluorescence imaging of Dictyostelium discoideum with a hard X-ray nanoprobe. Journal of Physics: Conference Series, 186(1):012086, 2009.

[87] A. C. Thompson, J. Kirz, D. T. Attwood, E. M. Gullikson, M. R. Howells, Y. L. Kortright, A. L. Robinson, J. H. Underwood, K.-J. Kim, I. Lindau, P. Pianetta, H. Winick, G. P. Williams, and J. H. Scofield: X-ray Data Booklet. Center for X-ray Optics and Advanced Light Source, Lawrence Berkeley National Laboratory University of California Berkeley, CA 94720, third ed., 2009.

[88] H. Iwамото: Quality evaluation of quick-frozen biological specimens by simultaneous microbeam SAXS/WAXS recordings. J. Synchrotron Rad., 16(3):336-345, May 2009.

[89] B. L. Henke, E. M. Gullikson, and J. C. Davis: X-Ray Interactions: Photoabsorption, Scattering, Transmission, and Reflection at $E=50-30,000 \mathrm{eV}, Z=1$-92. Atomic Data and Nuclear Data Tables, 54(2):181-342, July 1993. 


\section{Introduction}

Amoeboid migration is representative for the migration mode of many eucaryotic cells including immune cells and cancer cells [1-3]. It is of particular interest, as the migration is related to, e. g., the spreading of cancer cells in tissue. Basic insight into the biomechanical and biochemical processes underlying this migration is accessible from studying amoebae like Dictyostelium discoideum (D. discoideum). Many aspects of this mechanism have already been investigated:

- the structure of actin monomers, filaments, and many actin binding proteins was resolved by protein crystallography and small angle x-ray scattering (SAXS) on aqueous solutions $[4,5]$,

- the structure of actin filaments, actin binding proteins, and actin networks by cryoelectron microscopy,

- signalling pathways, which link the concentration of the chemoattractant cyclic adenosine monophosphate ( $c A M P$ ), detected by extracelluar receptors, to intracellular proteins and regulate the polymerization of actin filaments in a certain region of the $D$. discoideum cells $[6,7]$,

- the spatiotemporal dynamics of cell wall curvature and the concentrations of the proteins Ras and LimE allow to conclude on the interdependence between the molecules and the influence of the membrane shape [8], and

- the dynamics of actin polymerization in vitro in the presence and absence of actin binding proteins [9].

Yet unknown is the structure of the actin fibre bundles in the cell and the structural changes during the migration of the amoeba. We studied the structural properties in (D. discoideum) by means of scanning small angle x-ray scattering with nanobeams (nano-SAXS), which is capable of overcoming limitations of the methods used previously on $D$. discoideum. By using nanometre sized beams we probe the sample structure in a precisely controlled region of the cell without information loss due to averaging, which is common to conventional SAXS experiments. The invasive sample preparation protocols 
required for electron microscopy (EM) can be avoided, as the high penetration depth of $\mathrm{x}$-rays does not require slicing or (osmium) staining of hydrated samples.

Small angle x-ray diffraction is capable of resolving structural parameters of the fibre bundles in the cells, like the fibre bundle thickness, orientation, and, possibly, internal structure. Weinhausen et al. $[10,11]$ successfully applied nano-SAXS to cells derived from cancerous human adrenal cortex cells, that form bundles of the intermediate filament keratin. Scanning nano-SAXS on freeze-dried, chemically fixed, and living cells allowed the group to map the scattering intensity and orientation as a function of the position in the cell.

Recent developments in coherent diffractive imaging (CDI) now allow phase contrast imaging from freeze-dried and frozen-hydrated samples under cryogenic conditions [12-15], but these methods do not yet reach the resolution found in diffraction experiments.

We applied nano-SAXS to unstained and unsliced frozen-hydrated samples of single D. discoideum cells and complemented this approach with optical cryo microscopy, fluorescence microscopy, x-ray fluorescence microscopy, and first results from ptychographic reconstructions on frozen-hydrated specimens of $D$. discoideum.

After this introduction, chapter 2, Fundamentals, will give a background on the D. discoideum cells and the actin filaments and networks inside and introduce the experimental techniques applied.

The following section on materials and methods, chapter 3, introduces the cell lines, cell culture, preparations protocols, sample environments, and x-ray setups.

A novel anisotropy parameter, that we recently introduced to a greater audience [16] and nicknamed "Streak Finder", is defined in chapter 4. A new normalization is introduced and simulations with artificial data are used to characterize the anisotropy parameter, before a comparison to existing definitions is drawn and an outlook on possible adjustments is given.

The results from our measurements are presented in chapter 5, where they are grouped into results from the interpretation of reciprocal space and real space, additional aspects covered are a simulation of fibre diffraction data, electron density maps from ptychographic reconstructions, and elemental maps obtained from x-ray fluorescence aquisitions.

Finally, the results are summarized and an outlook is given in chapter 6 .

Matlab scripts employed for the data analysis and manuals for the instruments used and commissioned follow in the appendix. 


\section{Fundamentals}

\subsection{Dictyostelium discoideum}

D. discoideum is an amoeba that belongs to the genus of slime molds (amoebozoa) [17]. In nature, $D$. discoideum normally live as single cells in the soil and feed from bacteria by phagocytosis. Upon depletion of nutrients, the cells enter a starvation cycle during which they try to aggregate and form a multicellular organism, the so-called slug. The slug is able to migrate over larger distances to the soil surface before it finally differentiates into a spore body, which is carried by a thin stalk. After releasing the spores, the stalk cells die and the spores have a chance to settle in a different spot. This tactic increases the probability for the colony survival [18]. Hence, these cells neither strictly belong to the unicellular nor to the multicellular category of organisms [19]. Remarkable is, that spore and stalk consist of different cell types that developed from the same D. disoideum cells by differentiation.

To gather a colony by aggregation, the cells start to release the signaling molecule cyclic adenosine monophosphate (cAMP). They simoultaneously sense and actively follow the gradient of cAMP concentration in order to find its source, i. e., other D. discoideum cells. This behaviour is called chemotaxis. In addition, the cells show hapto-, magneto-, aero-, photo-, thermo-, duro-, mechano-, rheo-, electro-, and curvotactic behaviour [3, 18, 20,21]. Chemotaxis enables the specimens to compare chemical concentrations at different points along their cell surface, to translate these information into directional information and finally to migrate in a directed motion. Three steps are required for chemotactic behaviour [22]: Motility is a characteristic shared by starved D. discoideum cells even in the absence of external stimuli. Gradient sensing can trigger directed migration and propulsion of pseudopods (i. e, a projection of the plasma membrane outwards of the cell). Finally, cells can undergo a polarization (even in homogeneous concentrations of a chemoattractant) which restricts the neoformation of pseudopods to the cell front.

Amoebae move by projecting the cell membrane outwards and forming adhesions to the substrate. A cell can pull on these adhesions and retract its rear end by a combination of actin-polymerisation and myosin-contractility. In strong gradients, D. discoideum cells follow a cAMP gradient directly by propelling the pseudopodium at the front side 
and retracting the rear end. For shallow gradients (which are typically found during the initiation stage of the aggregation process), a tip splitting mechanism is observed in D. discoideum during which new pseudopods are formed alongside existing ones, often alternating the sides every time, resulting in an "ice scating" like motion $[22,23]$. The protrusions stay attached to the substrate as the amoeba migrates, i. e., the pseudopodia run along the cell's perimeter. Sensing a gradient direction different from the pseudopods orientation can result in the retraction of a pseudopod [24].

The amoeboid migration process is mediated by controlled rearrangement of the cytoskeleton which determines the dynamics of the cell shape. The pseudopodia are protrusions of the plasma membrane, that are pushed outwards locally by polymerizing actin filaments (treadmilling [6,25-27]). In addition to pseudopod formation, the contractile retraction of the actomyosin network, a network of actin filaments with non-muscle myosin-II motor proteins, was identified at the cell's rear end in migrating $D$. discoideum $[27,28]$.

D. discoideum is a model organism for many effects and its motility is of particular interest, as the insights gained into this system may help to understand the migration of many eucaryotic cells such as immune cells (neutrophils, leukocytes) or cancer cells [1-3] that show a very similar behaviour. The migration of $D$. discoideum has been addressed on many different length and time scales and with different techniques:

Milimetre scale The nonlinear dynamics of cellular aggregation leads to the formation of spiral and concentric waves in the petri dish before the cells form the slug as well as inside the multicellular organism right before the mound is formed from which the stem grows $[29,30]$. Macroscopic behaviour can be observed on a length scale larger than a typical cell diameter of $10 \mu \mathrm{m}$ and has succesfully been described in terms of a reaction-diffusion model, the dynamics are presented in [31].

Micrometre scale The behaviour of single $D$. discoideum cells is well resolved in optical microscopy (especially in phase contrast or differential interference contrast or comparable methods). The reaction to different external stimuli like chemical gradients of cAMP can be tested [32-34].

The influence of gene inactivation (knockout of genes) on the cell behaviour helps to understand which proteins are involved in, e.g., the cell migration. By stable transfection with genes, which encode fluorescent markers attached to a target protein, the cells express fluorescently labelled proteins. Fluorescence microscopy allows to track the concentration of these proteins time dependent, while, e.g., external triggers are applied. 
These manipulations are well handable in D. discoideum, as it is a eucaryotic, haploid (i. e., it carries one copy of the genome) cell which has been fully sequenced [17].

Nanometre scale Electron microscopy resolves single filaments of actin in real space. It is used to investigate the structure of single filaments up to the arrangement of filaments in the actin networks $[35,36]$. In vitro study on the structure of polymerized or polymerizing actin networks can answer the question how these networks self organize without external factors and how additional bundling proteins influence the physical properties of the networks $[9,37,38]$.

Microsecond scale Besides many orders of magnitude in lengths, the time scales are also important: When starved, D. discoideum are moving at a speed of $\approx 10 \mu \mathrm{m} \mathrm{min}^{-1}$, which is on the order of one cell length per minute and relatively fast for migrating cells. During this time, several rearrangements in the cytoskeleton are taking place and the leading edge of the cell tips even reach velocities of $\approx 1 \mu \mathrm{m} \mathrm{s}^{-1} \ldots 5 \mu \mathrm{m} \mathrm{s}^{-1}$ [39]. The temporal resolution is crucial, e.g., for studying the correlation between membrane curvature and concentration of different membrane bound signalling proteins in the context of pseudopod dynamics. This aspect is currently being addressed by Christoph Blum and coworkers ${ }^{1}$ in collaboration with the group of Jörg Enderlein ${ }^{2}$. The required temporal (and spatial) resolution is achieved by using an ultrafast version of confocal spinning-disk image scanning microscopy [40] and allows conclusions on the pattern formation in the actin cortex of chemotacting cells [8].

The above examples illustrate the required spatial and temporal resolution of imaging techniques that are developed in this work. A broad knowledge of the molecules involved in the migration and chemotaxis has already been gained, but the detailed understanding of how the signal detection leads to a directed motion is still elusive.

Nanobeam scanning SAXS can help here to quantify the local structural properties of actin networks, as it probes only a small region (on the order of down to $100 \mathrm{~nm} \times 100 \mathrm{~nm}$ ) and yields structural information on length scales of $\approx \mathrm{nm}$. The probe size corresponds to length scales on which the filament networks change in their conformation [41] and the far-field intensity distribution resolves details on the nano-structure of filaments. Nanobeam scanning SAXS therefore gives access to quantitative structural information on molecular to mesoscopic scales. This technique is thus well suited to help understand the physics and biology behind migratory processes. A deeper insight into the processes

1 Bodenschatz lab, Max Planck Institute for Dynamics and Self-Organization, Göttingen

2 III. Institute of Physics, Georg-August-University Göttingen 
involved in migration can be gained in combination with models, which connect the intracellular signalling cascade to observable dynamics at active sites of the cell [21].

\subsection{Actin}

Along with microtubules and intermediate filaments, actin is one of the three major constituents of the cytoskeleton found in all metazoan cells [26]. Together with a family of molecular motors, the myosins, it can generate strain and stress forces that are used for cellular processes like intracellular transport, cytokinesis, force generation in muscles and cell migration. The last point involves mechanisms like formation of pseudopods by actin networks as well as the retraction of the rear of a cell, also referred to as uropod [42], by contraction of an actin and myosin-II network situated below the cell membrane (so-called actomyosin cortex). These two features will be addressed later.

\subsubsection{Actin Structure}

The actin networks and bundles found in cells consist of single filaments (F-actin) which are polymerized from the monomeric actin molecules, so-called globular actin (G-actin). The G-actin protein is build from a single aminoacid chain with 375 aminoacid molecules and a mass of $42 \mathrm{kDa}$. Different representations of monomer and filament are shown in Fig. 2.1 (a-e). As clearly visible in the sketch, the molecule has an aspect ratio of approximately 1:2:2 and shows a cleft, in which an ATP- or ADP-molecule binds prior to polymerization. Actin binds to a divalent ion, under physiological conditions this is often $\mathrm{Mg}^{2+}[27]$.

The actin molecules remain monomeric in salt free buffers and begin to polymerize with increasing salt concentration. Monomers aggregate in a multistep polymerization process to oligomers (typically trimers), which then form filaments $[27,43,44]$. The actin filament structure depicted in Fig. $2.1(d)$ is generally agreed on, even though slight differences are found for example if other molecules are involved [45]: Five actin molecules are shown, arranged as found in a filament. The molecules are drawn in surface representation (i.e., an estimate on the volume required by the molecule, rendered from the Protein Data Bank information by the chimera software [46]). The G-actin monomers are arranged in a double helix, in which the molecules retain their orientation with respect to the filament axis. This results in a directionality of the filaments with a pointed and a barbed end.

Within growing actin networks, filament growth happens primarily by polymerization of ATP-bound G-actin on the barbed end of F-actin, as can be seen from the rate 
constants of actin polymerization, see Tab. 2.1. After binding, the ATP is hydrolysed by the actin molecule at a rate of $v_{\text {ATPase }}=0.3 \mathrm{~s}^{-1}$. The ADP bound actin molecules are depolymerized at the pointed end of the filaments. The ATP-actin polymerization at the barbed end corresponds to an elongation rate $^{3}>5 \mu \mathrm{ms}^{-1}$, which is half a typical cell diameter and thus much faster than typical migration speeds of $D$. discoideum. These elongation speeds have already been observed inside cells $[27,47]$.

\begin{tabular}{|c|c|c|c|c|}
\hline & \multicolumn{2}{|c|}{ ATP-actin } & \multicolumn{2}{|c|}{ ADP-actin } \\
\hline & Barbed & Pointed & Barbed & Pointed \\
\hline$k+\left(\mu^{-1} \mathrm{~s}^{-1}\right)$ & 11.6 & 1.3 & 3.8 & 0.16 \\
\hline$k-\left(\mathrm{s}^{-1}\right)$ & 1.4 & 0.8 & 7.2 & 0.27 \\
\hline
\end{tabular}

Table 2.1.: Rate constants for actin polymerization $k+$ and depolymerization $k$ - at the pointed and barbed end of filaments, from [47].
A cristallographic unit cell consists of the two half strands of the helix from one cross over to the subsequent and includes 13 molecules which make six turns $[9,48]$. This means that subsequent molecules are rotated by $\approx 166^{\circ}$ (in negative right handed rotation sense, i. e., the helix appears as a right handed thread), as illustrated in Fig. 2.1. The periodicity in filament direction is $36 \mathrm{~nm}$ corresponding to an increment of $2.8 \mathrm{~nm}$ per molecule respectively [49]. The periodicity is very sharply peaked while the incremental rotation between neighbouring molecules allows a slight deviation, values between $\pm 1^{\circ}[9]$ and $\pm 10^{\circ}$ [50] have been reported. The persistence length of an actin filament is $L_{\mathrm{P}}=17(2) \mu \mathrm{m}$ [51] which is on the order of the typical length of a cell. This stiffness ranges between the microtubules which reach values of two orders of magnitude larger [51] and, e.g., Keratin, which belongs to the intermediate filaments and has a persistence length of $L_{\mathrm{P}} \approx 1 \mu \mathrm{m}$ [52].

\subsubsection{Actin Networks and Actin Binding Proteins}

Actin binding proteins (ABP) are a large group of proteins (over 160 [54]) that interact specifically with actin and can induce, e.g., the bundling and severing ("cutting") of F-actin, binding of new filaments to an existing one (so-called "branching"), inhibition of aggregation at the barbed end, or the increasing of the turnover of actin [44].

Bundles in solutions of F-actin with one crosslinker do not exceed a number of $\approx 20$ single filaments per bundle, a mechanism based on the helical structure of the filaments makes this energetically unfavourable, as shown by Claessens et al. [9], based on experiments on fascin/actin mixtures. Additional actin binding molecules of a different species lead to further growth of the bundles. In actin bundles, the filaments are arranged in a hexagonal lattice with a lattice spacing of $D_{h e x}=12.4 \mathrm{~nm}$, as obtained from SAXS measurements on actin filaments with the crosslinker fascin [9].

$3 \quad$ For a G-actin concentration of $c_{\mathrm{G}-\text { actin }}=300 \mu \mathrm{M}$ and ATP-bound G-actin with $k+=11.6 \mu^{-1} \mathrm{~s}^{-1}$ $[27]$. 


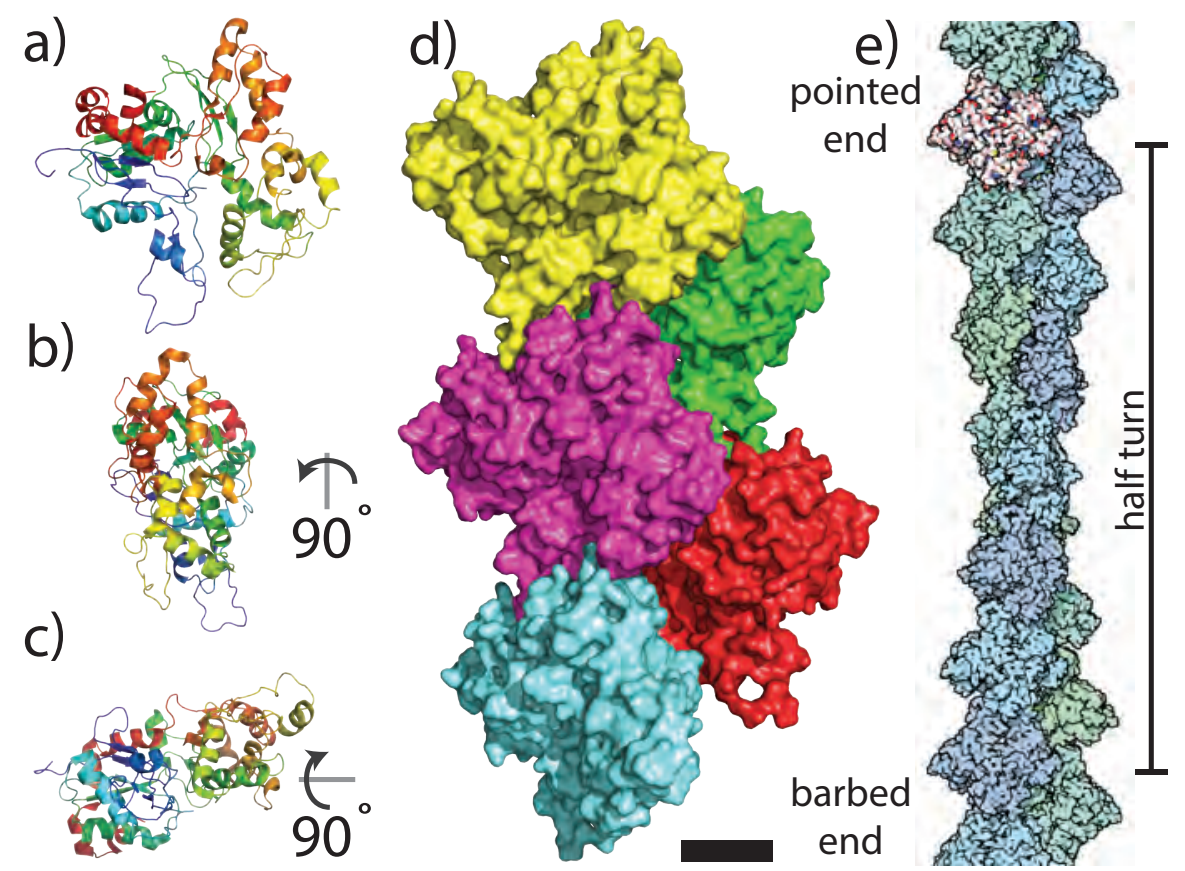

Figure 2.1.: Different representations of actin molecules. (a) Single actin molecule in "cartoon" representation (The aminoacid chain is drawn as a solid line, with $\alpha$-helices and $\beta$-sheets represented as helices and flat arrows respectively. The arrow heads on the beta sheets point toward the c-terminus of the aminoacide chain. The color code of the molecule corresponds to the position in the amino acid sequence starting from the $\mathrm{n}$-terminus in darkblue toward the c-terminus in darkred.). (b) shows the same molecule from (a) rotated $90^{\circ}$ around the vertical axis to the left, (c) shows the same molecule from (a) rotated $90^{\circ}$ around the horizontal axis now facing the lower side to the front. (d) shows a "surface" representation of five actin molecules, arranged as found in a polymerized actin molecule. The filament axis goes along the vertical axis in the image. The purple molecule has the same orientation as the one in (a). The scale bar depicts $2 \mathrm{~nm}$. The images are drawn with PyMOL software [53] (a)-(c) and USCF chimera software [46] (d), structure data are taken from the Protein Data Bank entry ID 4a7n [45]. The structure is obtained from rabbit skeletal muscle actin by cryo electron microscopy of vitrified samples. (e) Longer segment of $\mathrm{F}$-actin in surface representation (taken from Protein Data Bank [5], Molecule of the Month July 2001, last accesssed 30.11.2014, see doi: $\left.10.2210 / \mathrm{rcsb}^{\circ} \mathrm{pdb} / \mathrm{mom}^{\circ} 2001^{\circ} 7\right)$. The bar on the right side denotes one half turn of actin, which measures $36 \mathrm{~nm}$, in (d,e) the barbed end is facing down and the pointed end up. 
Actin network in pseudopods Membrane protrusions like the pseudopods, found in $D$. discoideum, are pushed out by filamentous polymerization of local actin networks. These networks consist of highly crosslinked actin filaments towards the cell centre, controlled by the branching protein Arp2/3 and the WAVE/WASP complex. Single filaments of actin are found near the growing end of the pseudopod close to the membrane [36]. The filaments elongate by polymerisation at the membrane side of the network. Towards the cell nucleus, the networks depolymerize and thus release G-actin, which is then transported to the cell membrane by diffusion. This process is referred to as "treadmilling".

The Arp2/3 complex occurs increased at the side of a chemotactic stimulus, it plays a role in the regulation and formation of pseudopods and itself is upregulated by WASP [55]. Arp2/3 attaches to the side of existing filaments and enables the nucleation of oligomers on the side of a filament. The barbed ends of both filaments grow away from the branching point with a fixed angle of $\approx 70^{\circ}$ in between. The F-actin is consequently oriented with the barbed end towards the membrane. Myosin motor proteins are not found in growing pseudopods, they only occur on the sides of the protrusion region and in the uropods, see Fig. 2.2 (a). Cryo electron microscopy imaging on lamellipodia, however, could not yet confirm the predominant occurence of a characteristic angle between filaments [36]. The authors suggested a three dimensional cryo electron microscopy to put this hypothesis to test and prevent preparation artefacts from a fixation protocol involving critical point drying. A later study confirmed that inhibition of Arp2/3 results in a reduced branching frequency [7] - indicating that the Arp2/3 is involved in the formation of the lamellopodium (a pseudopod related membrane protrusion found in many eucaryotic cells).

Actomyosin cortex Below the cytosol membrane of $D$. discoideum, a network of actin with non-muscle myosin II is found, that is linked to this membrane. The actin filaments of this network are oriented in parallel to the membrane surface (see also electron micrograph from cortex region in [57], Fig. 7). It is involved in cellular motility, where it contracts around the rear end of the cell and thus pulls the cell forward. Myosin II consists of two motor domain heads connected by a linker. When both migrate towards the barbed end of the filaments, the filaments are slided relatively against one another. As a result, actomyosin networks contract, when myosin II is actively moving. In contrast to the pseudopodium, the filaments are not oriented in parallel, but randomly within the plane below the membrane. This process was observed in fluorescence microscopy of $D$. discoideum with labelled myosin, where a so-called "C-to-spot" [28] translocation of the contracting actin-myosin II network is visible in the uropod. "C-to-spot" refers 


\section{a) F-actin / MyosinII}

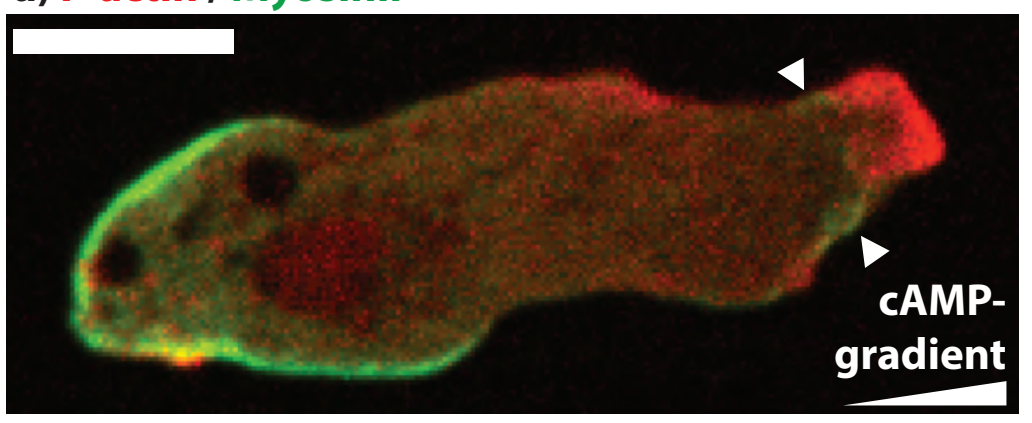

\section{b) Scheme}

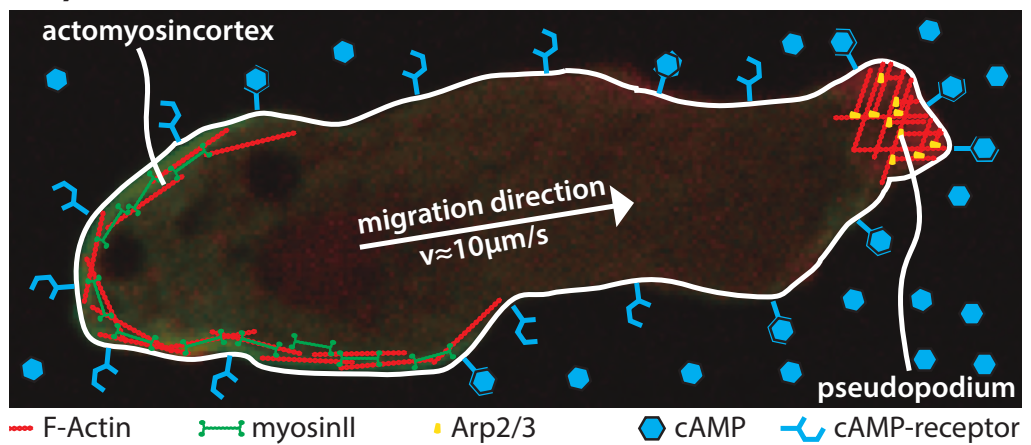

Figure 2.2.: Image a) and sketch b) of D. discoideum cell. Fluorescence micrograph of a single moving $D$. discoideum, that is labelled with a mRFP on the LimE-group (in red) and a GFP on the myosin-II (in green). The cell moves to the right side within a linear concentration gradient of the chemoattactant $c A M P$. The gradient points in the horizontal direction towards the right image side, where the concentration is maximal. The myosin II signal is strong in the actomyosin network (see sketch in b)) on the left side, where the uropod is situated, but the sides of the pseudopod (to the right side) show increased intensity from myosin II as well (see indicators in a)). The pseudopodium at the leading edge of the cell shows high intensity in F-actin. Receptors on the membrane allow to detect the concentration of cAMP in the environment of the cell. The cell was flattened in a specialised device [56] and appears therefore larger than usual. Scale bar denotes $10 \mu \mathrm{m}$. Image aquired with an Olympus IX81 Confocal Laser Scanning Microscope. Image courtesy of Marco Tarantola, AG Bodenschatz, MPI Biophysikalische Chemie, Göttingen. 
to the optical appearance in the microscope, where the arc-shaped actomyosin network contracts to a spot. A similar behaviour is observed in cell cleavage during cytokinesis, when a contracting ring of actomyosin pinches the membrane to form the cleavage furrow where the two new daughter cells separate.

More proteins are involved in the regulation and formation of pseudopods and the structure of the actin network. Scanning SAXS with nano beams can contribute here by testing structural properties on the nm scale while averaging over a region of the beam size, i. e., $100 \mathrm{~nm}$. Also, possible artifacts from the preparation for electron microscopy (like drying and staining, [36]), but also for cryo electron microscopy (e.g., slicing) are avoided, since cells can be investigated in their native environment or exposed to controlled stimuli. 


\subsection{Scanning Transmission X-ray Microscopy}

The basic theory behind scanning transmission x-ray microscopy (STXM) is well established and can be found in many textbooks [58-60]. This section is based on the monograph by Paganin [59] and will briefly introduce the relations used in the present work. I will therefore stick with the nomenclature used by Paganin and recommend it for more in-depth consideration of the topic.

In x-ray scattering, incoming x-radiation impinges on a sample and the scattered radiation is recorded in the far-field regime (Fraunhofer diffraction). The Fresnel number $N_{F}=\frac{b^{2}}{\lambda L}$, where $b$ denotes the beam diameter (for the case of nano beams), $\lambda$ the wavelength and $L$ the sample to detector distance, can act as a measure and should be well below $N_{F}<<1$ to ensure Fraunhofer diffraction [59]. Multiple scattering processes are neglected. The interaction of the incoming wave field $\psi_{i n}(\mathbf{x})$ (without time dependent part) is modelled by a multiplication with a term accounting for the phase shift $\delta_{n}(\mathbf{x})$ and absorption $\beta_{n}(\mathbf{x})$ inside the sample. These are related with the complex refractive index $n(\mathbf{x})$ :

$$
n(\mathbf{x})=1-\delta_{n}(\mathbf{x})+\imath \beta_{n}(\mathbf{x}),
$$

with $\delta_{n} \approx 10^{-5}$ and $\beta_{n}$ even smaller for the case of hard x-rays [58].

For a plain wave with wave vector $\mathbf{k}=\frac{2 \pi}{\lambda} \hat{\mathbf{k}}$ (where $\hat{\mathbf{k}}$ denotes the unity vector of $\mathbf{k}$ ) propagating in $\hat{\mathbf{x}}$-direction $\mathbf{k}=k \hat{\mathbf{x}}$, the exit wave field reads

$$
\psi_{\text {ex }}=\exp \left(-\imath k \int_{x_{\text {sample }}}\left(\delta_{n}(\mathbf{x})-\imath \beta_{n}(\mathbf{x})\right) \mathrm{d} x\right) \cdot \psi_{\text {in }}(\mathbf{x})
$$

with the integral over $x_{\text {sample }}$ the thickness of the sample. This is often referred to by projection approximation.

The lateral variations in the integrated phase shift in horizontal $(y)$ and vertical $(z)$ direction

$$
\frac{\partial}{\partial_{y}} \Delta(\varphi)=-k \frac{\partial}{\partial_{y}} \int_{x_{\text {sample }}} \delta_{n}(\mathbf{x}) \mathrm{d} x
$$

( $z$-direction accordingly) result in a deflection of the (primary) beam. The primary beam position on the detector is determined for every diffraction pattern and is used to plot a so-called differential phase contrast (DPC) map of the sample. For practical reasons the components in the lateral directions are plotted separately, as indicated with an index $\mathrm{DPC}_{y}$ or $\mathrm{DPC}_{z}$. The absolute scale of these images is usually arbitrary and often difficult to assess due to (semitransparent) beamstops, the maps are therefore plotted in units of pixels. 
a)
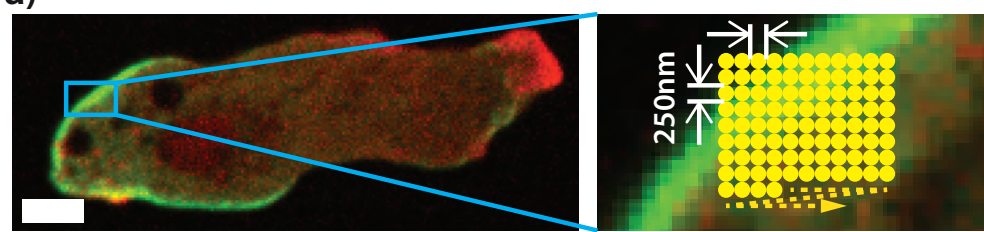

b) Composite

I/ph./s $10 \quad 100 \quad 1000$

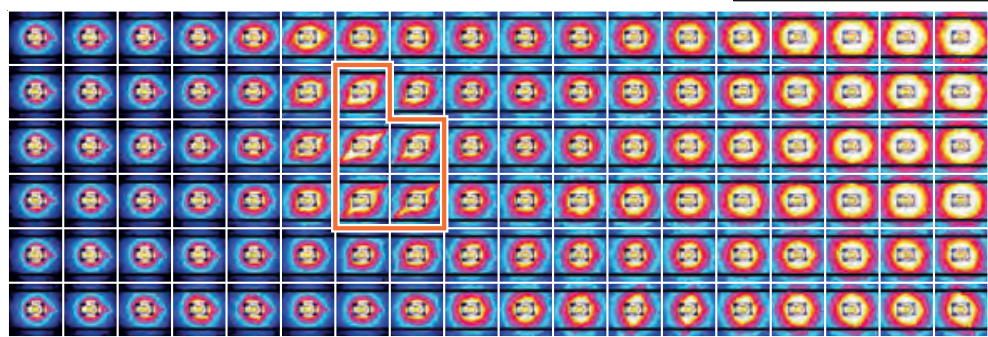

c) darkfield I

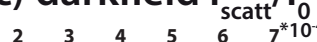

\begin{tabular}{llllll}
2 & 3 & 4 & 5 & 6 & $7^{*} 10$ \\
\hline & & & $\vdots$ & &
\end{tabular}

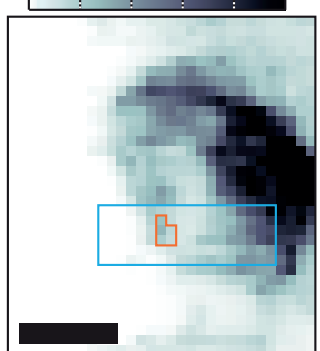

d) DPCy / Pixel

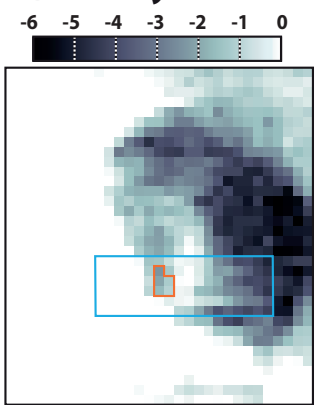

e) anisotr. param.

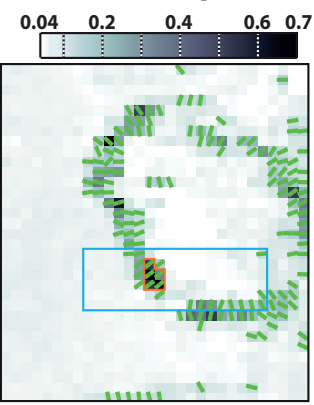

Figure 2.3.: Illustration of scanning nano-SAXS, collage of a corresponding typical dataset for overview and generated contrast maps of same dataset. (a) Sketch of the rastering scheme for nano-SAXS: A part of the D. discoideum cell shown in Fig. 2.2 (a) is overlayed with circles representing the diameter (FWHM) of the beam focus (here $250 \mathrm{~nm}$ ). The arrangement of the circles shows the rastering scheme of a fine mesh scan. (b) Collage of the far-field intensity distributions arranged according to where the beam impinged the cell. This subset corresponds to the blue rectangle in panels (c-e), and its orange region corresponds to the one in panels (c-e). All far-field images are oriented as seen with the beam, i. e., left is positive y-direction and up the positive z-direction (as refered to the axes in Fig. 3.3 (a)). A single diffraction pattern was obtained with a dwell time of $0.1 s$. Adjacent diffraction patterns were recorded $500 \mathrm{~nm}$ apart. (c-e) Quantified maps from the same dataset: (c) The darkfield map color encodes the scattered intensity with respect to the primary intensity of the scan. A cell can be identified by more intense scattering. (d) The differential phase contrast in horizontal direction, calculated from the deviation of the centre of mass in the diffraction pattern. (e) Anisotropy coefficient as described in Fig. 4.1: Highly oriented intensity distributions in the far-field occur on the rim of the cell, the orientations of the patterns shown are standing perpendicular to the cell cortex indicating structures in the cell with a high orientation parallel to the cell cortex. All scale bars equate $5 \mu \mathrm{m}$. Material of this figure and caption was also published in [16]. 
The intensity, i. e., the absolute of $\psi$ to the power of two is Lambert-Beers law for absorption, when $2 k \beta=\mu$ is replaced:

$$
I_{e x}(\mathbf{x})=\exp (-\mu T(y, z)) I_{i n}(\mathbf{x})
$$

where $T(y, z)$ denotes the projected thickness of the sample in $z$ direction. Maps of the transmission $t(y, z)=\frac{I_{e x}(y, z)}{I_{i n}}$ suffer from the same challenges as the calculation of the absolute DPC, also the transmission of thin biological samples is very close to one. As the cells are by far more clearly visible in the other contrast maps, the transmission is not used in this work.

The wave vector is introduced as the difference between the wave vectors of the incoming $\mathbf{k}_{\mathbf{i}}$ and the diffracted $\mathbf{k}_{\mathbf{f}}$ (

$$
\begin{aligned}
\mathbf{q} & =\mathbf{k}_{\mathbf{f}}-\mathbf{k}_{\mathbf{i}}, \text { with } \\
q & =\frac{4 \pi}{\lambda} \sin \left(\frac{2 \theta}{2}\right)
\end{aligned}
$$

where $2 \theta$ denotes the angle between $\mathbf{k}_{\mathbf{i}}$ and $\mathbf{k}_{\mathbf{f}}$. For a given electron density distribution $\rho_{e^{-}}(\mathbf{x})$ the field distribution in the far-field

$$
\psi(\mathbf{q}) \propto \int_{\mathbf{x}} \rho_{e^{-}}(\mathbf{x}) \exp (-\imath \mathbf{q} \mathbf{x}) \mathrm{d} \mathbf{x}
$$

can be rewritten as the Fourier transform of $\psi, \tilde{\psi}$, which yields for the intensity

$$
I(\mathbf{q}) \propto\left|\tilde{\psi}\left(\rho_{e^{-}}(\mathbf{x})\right)\right|^{2}
$$

The scattered radiation carries structural information about the projected electron density distribution in the sample. Contrast mechanisms like the darkfield or orientation parameter can be derived, but the structural information of the diffraction patterns can be accessed as well. The darkfield contrast is calculated from the integrated scattered intensity, i. e., number of scattered photons, divided by the intensity of the primary beam. Chapter 4 gives detailed information on the order parameter mentioned. 


\section{Materials and Methods}

In this chapter the cell lines used in this work are introduced, followed by the preparation protocols and sample environments. The last section will address the SAXS setups and x-ray techniques employed. All cells used were generously supplied by the Bodenschatz group $^{1}$ as part of a close collaboration between the groups of Bodenschatz, Enderlein und Salditt in the context of the collaborative research centre 937.

\subsection{Cell Lines}

All cell lines used in this work are based on the axenic cell lines, which do not feed upon Escherichia coli (E. coli) bacteria, that grow in the medium, but from nutrients diluted in the medium. Apart from the wild-type cells we had genetically modified strains with fluorescent markers. Green fluorescent protein or red fluorescent protein are attached to either myosin-II motor proteins or to LimE-proteins [61]. Both fluorescent dyes are commonly used markers that can be recorded at most fluorscence microscopes. LimE is a component of the Arp2/3-complex that is included in actin networks during polymerization, LimE with fluorescent labels thus selectively mark filamentous actin polymerization [39].

D. discoideum AX2-wt denotes the wild type of the axenic stem AX2 [62]. These cells are derived from the first axenic $D$. discoideum stem reported and provided to the Bodenschatz lab by the group of Günther Gerisch ${ }^{2}$. All following cell lines are derived from this one by genetical modification.

D. discoideum AX2-LimE-GFP is a strain with a green fluorescent protein (GFP) fused to the LimE-protein, to selectively label polymerized actin.

D. discoideum AX2-LimE-mRFP-Myoll-GFP has a double label, the LimE-protein carries a monomeric Red Fluorescent Protein and a GFP is fused to the myosin-II

1 Max-Planck-Institut für Dynamik und Selbstorganisation, Göttingen

2 Max Planck Institute of Biochemistry, Martinsried, Germany 
motor proteins. This strain allows the visualization of the actomyosin cortex (which contains myosin-II) and the pseudopods (purely polymerized actin without myosin) quasi simultaneously by subsequently recording two flurescence channels on the same cell.

D. discoideum AX2-LimE-GFP-Myoll-null cells carry a GFP label on the LimE as for the D. discoideum AX2-LimE-GFP, but in addition, the myosin-II-motor proteins are not expressed, the encoding gene is knocked out. The typical contractile arc, that is formed by actin together with these myosin-II-motor proteins can therefore not be formed. This knockout is a way to test a hypothesis that involves myosin-II.

\subsection{Cell Culture}

The cell culture is routinely done in the lab of Eberhardt Bodenschatz, which generously supplied the cells whenever we needed them. As I never cultured the cells on a regular basis, I will focus on the very basic procedures, further information can be found in [32,63].

\subsubsection{Buffers and Media}

D. discoideum is grown in the (axenic) $\mathbf{H L 5}$ medium, which contains $7.0 \mathrm{~g} / \mathrm{l}$ yeast extract, $14.0 \mathrm{~g} / \mathrm{l}$ protease peptone, $0.5 \mathrm{~g} / \mathrm{l} \mathrm{KH}_{2} \mathrm{PO}_{4}, 0.5 \mathrm{~g} / \mathrm{l} \mathrm{Na}_{2} \mathrm{HPO}_{4} \cdot \mathrm{H}_{2} \mathrm{O}$ and $13.5 \mathrm{~g} / \mathrm{l}$ glucose. The dry mixture is delivered by ForMedium ${ }^{3}$ ), diluted in $\mathrm{MilliQ}^{4}$ and finally sterilized by autoclaving.

The starvation and washing of cells is done in the Phosphate Buffered Saline solution "Sorensen buffer" (short PBS), a solution of $14.7 m M \mathrm{KH}_{2} \mathrm{PO}_{4}$ and $2 \mathrm{mM}$ $\mathrm{Na}_{2} \mathrm{HPO}_{4}$ in MilliQ, that is adjusted to pH6.0 and autoclaved.

For chemical fixation of the cells, the formaldehyde fixation solution is used. A concentrated formaldehyde solution (37\% formaldehyde in water with $10 \%$ methanol $^{5}$ ) is diluted in PBS to a final formaldehyde concentration of $4 \%$.

A Triethylammonium acetate (TEAA) buffer (also-called volatile buffer) can be used to prevent the formation of salt residues upon freeze drying, as the ions of the buffer evaporate in vacuum. This preparation step is adapted from electron microscopy. TEAA stock solution ${ }^{6}$ needs to be diluted in MilliQ to the desired concentration. The Morse

$3 \quad$ Order No. HLG0101 by ForMedium, Norfolk, UK

4 MilliQ is a term for ultrapure water from a Milli-Q-system by Merck Millipore, a daughter company of Merck KGaA, Darmstadt, Germany. It is produced from tap water by filtering (pore size 220nm) and deionizing to a final resist of $18.2 \mathrm{M} \Omega \mathrm{cm}$.

5 Order no.1-04002.1000 by Merck KGaA, Darmstadt, Germany,

6 Order no. 90357 by Sigma-Aldrich Chemie GmbH, Steinheim 
equation (in German van-'t-Hoff'sches Gesetz) has to be considered in order to equilibrate the osmotic pressure $\Pi_{\mathrm{TEAA}}$ of the TEAA buffer to PBS $\Pi_{\mathrm{TEAA}}=c_{\mathrm{TEAA}} \cdot i_{\mathrm{TEAA}} \cdot R \cdot T$, with the molar concentration $c_{\text {TEAA }}$, the number of particles in solution per unit of substance $i$ (so-called van 't Hoff factor), the gas constant $R$, and the absolute temperature $T$. For the given case this can be reduced to the simple equation $i_{\mathrm{PBS}} \cdot c_{\mathrm{PBS}}=i_{\mathrm{TEAA}} \cdot c_{\mathrm{TEAA}} \cdot$

\subsubsection{Growing of Cells}

The cells are grown in (uncoated) petri dishes in nutrient medium and at a constant temperature of $22^{\circ} \mathrm{C}$. Every three days the cells need to be passaged, i. e., one drop of the cell suspension is transferred to a new dish with new medium.

\subsubsection{Active Preparation of Chemotactically Competent Cells}

These preparation steps are also done in the lab of E. Bodenschatz, the following protocol is taken from $[16,32]$ :

The preparation of chemotactically competent cells, so-called pulsing, starts one day prior to the experiment being carried out. An inoculum of $1 \cdot 10^{6}$ cells is pipetted into $25 \mathrm{ml}$ HL5 medium and cultivated at $22^{\circ} \mathrm{C}$ as a shaking culture (150 rpm). 7 hours in advance of the experiment the full shaking culture is centrifuged ( $1000 \mathrm{rpm}, 3 \mathrm{~min}, 4^{\circ} \mathrm{C}$ ), washed once in phosphate buffer (PBS, $p H 6)$ and the remaining pellet is diluted in $20 \mathrm{ml}$ PBS. This new shaking culture $\left(150 \mathrm{rpm}, 22^{\circ} \mathrm{C}\right)$ is subject to periodic pulses of 3'-5' cyclic adenosine monophosphate (cAMP, SigmaAldrich) in order to enhance the development of the $D$. discoideum amoebae [64]. A pulse of cAMP consists of approximately $60 \mu l$ with a concentration of $18 \mu M$ and is added every 6 minutes. The cells develop for 5 hours. 1 hour prior to further preparation, the cells are washed 1 to 3 times in PBS (1000 rpm, $3 \mathrm{~min}, 4^{\circ} \mathrm{C}$ ) and are finally dispersed in $10 \mathrm{ml}$ PBS (depending on the cell density).

Prepared by this means, the cells reach a state of high motility and can directly be used for further preparation steps. These cells were used for most of the frozen-hydrated, freeze-dried, and chemically fixed cells, samples with living cells could not be treated this way, as the pulsing is not feasible in preparation labs at synchrotron radiation experiments. 


\subsubsection{Passive Preparation of Chemotactically Competent Cells}

Especially if pulsing equipment is not available, cells can be made chemotactically competent by rinsing the cells off the petri dish and applying a given amount of the cell suspension onto a flat substrate or in a microfluidic channel. After allowing the cells to adhere for at least five minutes, the medium is carefully replaced with PBS (keeping in mind that shear forces can easily detach the cells). The cells enter the starvation cycle upon deprivation of nutrients, but not in a controlled fashion as achieved by pulsing. Regular optical inspection with a microscope is needed to ensure a high mobility of the cells before they can either be fixed or brought to the beamline for live experiments. Visual inspection with an inline microscope installed in the experimental hutch is inevitable when working with living cells to ensure that they are in the region of interest recorded during a scan.

If cells do not need to be in the best condition achievable, in terms of motility, pulsing is not necessary. For the investigation of living cells, motility and pseudopod formation shown by the cells were sufficient and happened reliably. This preparation method extends the period during which the cells are usable significantly and the cells are even becomimg more motile during the early experimental stage, as their maximum motility is still to come.

\subsection{Sample Fixation}

Biological cells suffer heavily from the exposure to synchrotron generated x-ray since it leads to heat impact and production of radicals in an aequeous environment. This results in structural changes, that are visible in the x-ray contrasts. As discussed in detail by [11], the produced radicals can migrate quickly within time scales of $\sim 1 \mathrm{~s}$. This is highly relevant for typical accumulation times of $0.05 \mathrm{~s} \ldots 0.5 \mathrm{~s}$ per scan-point used at the beamlines. The usage of fixation procedures enables longer exposure times. Different fixation procedures were applied to maintain the cells in their natural constitution during irradiation.

\subsubsection{Frozen Hydrated Cells}

This section 3.3.1 was also published in $[16]^{7}$.

Silicon nitride $\left(\mathrm{Si}_{3} \mathrm{~N}_{4}\right)$ membranes ${ }^{8}$ [(typical membrane size $1.5 \mathrm{~mm} \times 1.5 \mathrm{~mm} \times 1 \mu \mathrm{m}$ in

$7 \quad$ M. Priebe, M. Bernhardt, C. Blum, M. Tarantola, E. Bodenschatz, and T. Salditt: Scanning X-Ray Nanodiffraction on Dictyostelium discoideum. Biophysical Journal, 107(11):2662-2673, 2014.

$8 \quad$ Silson Ltd., Northampton, UK 

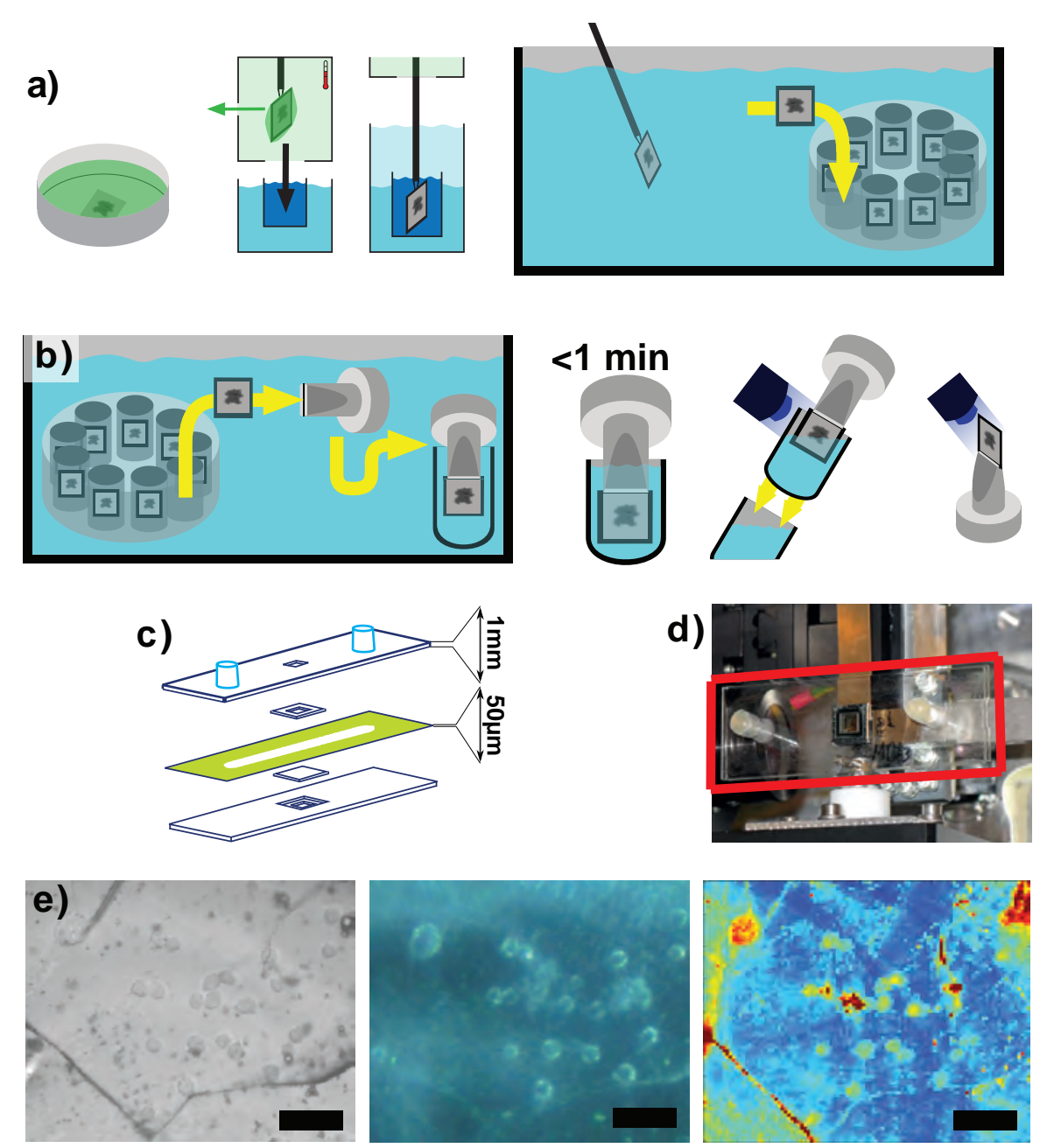

Figure 3.1.: (a) Illustration of cryoplunging process, (b) the mounting procedure of cryogenic samples, (c) schematic of a microfluidic chamber used for measuring initially living cells, (d) photograph of the transparent microfluidic chamber (outlined in red) installed at the GINIX. (e) Images of a frozen-hydrated sample: (left) Micrograph made with a cryogenic bright field light microscope in Köhler illumination. (middle) Same sample area as seen by the inline microscope at the GINIX setup in transmission illumination. (right) Corresponding x-ray darkfield contrast of same region. The scale bars correspond to $40 \mu \mathrm{m}$. Material of this figure and caption was also published in [16]. 
$5 \mathrm{~mm} \times 5 \mathrm{~mm}$ frames)] are rendered hydrophilic in a plasma cleaner for $10 \mathrm{~min}$ and are then placed in a petri dish with PBS. A drop of the cell suspension is gently pipetted onto the membrane and the cells are allowed to adhere, [Fig. 3.1 (a)]. As soon as the cells show a state of high motility, as inspected by optical microscopy (Zeiss Observer Z1), the membrane is transferred to the temperature and humidity controlled preparation chamber of a gridplunger (Leica GP2 [65-68]). A layer of PBS is always present on the membrane to prevent the cells from drying out.

Under inspection [by a] binocular microscope, the liquid is blotted inside of the preparation chamber followed by the fast injection into liquid ethane just above its boiling point $\left(-184^{\circ} \mathrm{C}\right)$. The fast cooling rate ensures vitrification of the water inside the cells. The samples are constantly kept at liquid nitrogen temperatures, thus well below the glass transition temperature value. The samples are brought to a storage vessel, where they are kept in a liquid nitrogen bath, see [Fig. 3.1 (a)].

[The solution can be used to reduce residues of salt on the freeze-dried samples. TEAA evaporates in high vacuum unlike the salts in Sorensen buffer and HL5 medium, the samples can be washed with TEAA right before cryo-plunging. The alternative is to wash the cells with demineralized water (e.g., ultrapure water as obtained from the Millipore system by Merck KGaA, Darmstadt, Deutschland). The osmotic pressure causes the cells to quickly swell and burst from it, so, if at all, this step had to be done very quickly , if it is at all feasible without interfering too much with the aspired non-invasiveness.]

\subsubsection{Freeze Dried Cells}

This section 3.3.2 was also published in $[16]^{9}$.

Freeze dried cells are prepared from frozen-hydrated cells, using a home built freeze-drier. The samples are passively cooled using a massive copper block installed at liquid nitrogen temperature in a large high vacuum vessel, over a time of 60 hours. The temperature of the sample slowly increases before the chamber is vented with dry nitrogen. After a further 12 hours under dry nitrogen atmosphere for slow temperature equilibration, samples are taken out. The samples are transferred to a vacuum desiccator to protect them from ambient humidity.

\subsubsection{Chemically Fixed Cells}

Living cells are allowed to adhere, either on a $\mathrm{Si}_{3} \mathrm{~N}_{4}$ membrane (typical membrane size $1.5 \mathrm{~mm} \times 1.5 \mathrm{~mm} \times 1000 \mathrm{~nm}$ ) in a petri dish (or well plate) or in a microfluidic channel

$9 \quad$ M. Priebe, M. Bernhardt, C. Blum, M. Tarantola, E. Bodenschatz, and T. Salditt: Scanning X-Ray Nanodiffraction on Dictyostelium discoideum. Biophysical Journal, 107(11):2662-2673, 2014. 
(see section 3.4.3 below). If the cells were not already washed and immersed in medium beforehand or even pulsed, it is necessary to wait for the cells to settle for at least $5 \mathrm{~min}$ before the medium is replaced with PBS. Next, the cells are incubated until they show high motility. PBS is then replaced with the formaldehyde fixation solution which is left on the substrate for 15 minutes. The samples are carefully rinsed with PBS twice and finally left under PBS. The samples are stored at $4^{\circ} \mathrm{C}$.

To prevent the formation of gas bubbles, the buffer should be replaced by degassed PBS. The following process proved to work best: PBS is filled into a vacuum safe glas bottle and placed into an ultrasonic bath. A vacuum pump (membrane pump, minimal pressure $\sim 10$ mbar $)$ is connected to the bottle. Ultrasonic bath and pump are operated simoultaneously for at least 15 minutes. For sterile working, a special filtration system ${ }^{10}$ can be used, which allows to first suck the unsterile buffer through a $0.22 \mu \mathrm{m}$ filter into the bottle, then close the air inlet and start the degassing procedure as described before $^{11}$. The procedure ensures sterile working even after longer working or storage time. However, the diffusion of gas molecules of the air through the containers and walls has to be considered when preparing the samples in advance.

\subsubsection{Living Cells}

These cells are prepared as described in chapter 3.2.4 and then transferred to a liquid chamber. The best results were achieved with ibidi chambers ${ }^{12}$. Sketch and photograph of an ibidi chamber are shown in Fig. $3.1(\mathrm{c}, \mathrm{d})$. A spacer of $50 \mu \mathrm{m}$ thickness is placed between two plastic slides that carry an x-ray compatible window (silicon nitride window). Two luer-ports allow to access the channel with a syringe. The plastic slides are relatively transparent for gases to facilitate cell culture in these chambers. For working with degassed liquids, this property is unfavourable, as gases dilute quickly in the liquid and bubble formation can be expected when exposed to the x-ray beam ${ }^{13}$. The gas dilution can be slowed down by keeping the chambers under degassed buffer after sealing.

The cell suspension is pipetted through the ports into the chamber, allowed to adhere for at least five minutes and rinsed with degassed PBS (see previous section for preparation instructions of degassed buffer). The channels need to be sealed carefully with plugs and can be used as soon as the cells migrate (typically after half an hour).

\footnotetext{
10 "Wiederverwendbare Bottle-Top Filterhalter" order no. KA90.1 by Carl Roth GmbH \& Co. KG, Schoemperlenstr. 3-5, 76185 Karlsruhe

11 This procedure was developed by my colleague Marten Bernhardt.

12 ibidi GmbH, Am Klopferspitz 19, 82152 Martinsried (München)

13 This was observed multiple times at differnt setups at our group already.
} 


\subsection{Sample Environments for X-ray Experiments}

\subsubsection{Cryostream}

The cryostream (700 series Cryostream Cooler by Oxford Cryosystems Ltd, Oxford, UK) is a gas stream cooler, that keeps a cryocooled sample at a defined temperature of $80 \mathrm{~K}$ or above. A copy of the setup and operation manual of the P10-Wikipage ${ }^{14}$ can be found in the appendix D.1. The essential cryostream system consists of the cryostream nozzle, a controller, and a supply dewar vessel, that provide a cryogenic gas stream. Dry gas is needed for a purge gas stream around the cryogenic gas. Therefore, an additional dry air unit (AD51) can be employed, or a hose with flowmeter is connected to a dry nitrogen gas source. In addition to that, an automatic refill unit can be operated, that pemanently monitors the liquid nitrogen level in the supply dewar vessel and regulates an automatic refill. A hose with electrically operated valve can be connected to a refill dewar (this needs to be pressurized as the refill system does not provide any pumping; see allowed pressures for the refill system), the valve is opened if the level in the supply dewar drops below a certain value (so-called setpoint), and it closes if it rises above a second setpoint.

The main difference to other approaches is that the sample remains in gaseous nitrogen under ambient pressure - there is no necessity to work in vacuum, which eases the sample change and other aspects significantly.

The cryostream provides a permanent cooling by a cold nitrogen gas stream. The central gas stream is surrounded by a second nitrogen gas stream at ambient temperatures, so-called purge stream. The purge stream ensures that no humid air contamines the central flow, which could result in the formation of water/ice on the sample and around the nozzle exit. Both, cryogenic and purge gas need to be free from humidity. The cryogenic gas stream is generated from liquid nitrogen, that is used for cooling and thus waterfree. At the GINIX instrument, gaseous nitrogen from liquid nitrogen tanks is used to provide gas for the purge gas stream. Alternatively, air can be dried (using, e.g., the $\mathrm{AD}$ drier unit from Oxford Cryosystems) and used for this purpose. As the AD51 unit is relatively noisy and operates a membrane compressor, the air stream it generates is pulsed and carries sound. To minimize vibrations in the system, we decided not to make use of the AD51 at GINIX.

The flow of cryogenic and purge gas are adjusted carefully to the specified flow rates, to ensure a laminar flow. Note that according to the manufacturer, the gas streams are laminar during normal operation. Assuming typical values for viscosity $\eta_{N_{2}} \approx 1 \mu \mathrm{Pas}$,

14 This is the internal help page for the GINIX instrument. Its home is the password encrypted website https://haspp10wg.desy.de/wiki. 
density $\rho_{N_{2}} \approx 10^{0} \mathrm{~kg} / \mathrm{m}^{3}$, speed $v_{N_{2}} \approx 10^{0} \mathrm{~m} \mathrm{~s}^{-1}$, and length scale $d \approx 10^{-3} \mathrm{~m}$ of the pipe, the Reynolds number for this setup ranges on the order of $R e=\frac{\rho v d}{\eta} \approx 10^{3}$. With the critical Reynolds number $R e_{\text {crit }}$ for the laminar to turbulent conversion in a tube $R e_{\text {crit }} \approx 2000$ (depending on the source, e. g., [69]), the cryostream tubing ranges already in a region, where some turbulence can be expected. The cryostream should thus be considered a low turbulence or nearly laminar flow, instead of a strictly laminar flow. Turbulence can be a source of vibration, and it causes mixing of the cryogenic gas, purge gas, and ambient athmosphere. Turbulence will thus result in transport of ambient humidity to the cryogenic flow region. This can be used to adjust the purge gas stream: The cryogenic gas stream is fixed at a flow rate of $\dot{V}_{\text {cryo }}=5 \mathrm{~L} \mathrm{~min}^{-1}$, the purge gas should be around $\dot{V}_{\text {purge }}=10 \mathrm{~L} \mathrm{~min}^{-1}$, but is best, when the formation of visible white fog in the stream is most distant from the nozzle exit ${ }^{15}$. This flow state is least disturbed by turbulence.

\subsubsection{Sample Mounting Procedure}

Although the mounting of cryogenic samples does not include a transfer into vacuum it has some challenges to it. If exposed to ambient air the ultracold samples easily accumulate ice by condensation and, even more critical, their temperature quickly rises above the glass transition temperature which causes the formation of crystalline ice. The mounting procedure for cryogenic samples is delicate, as the sample must never exit cryogenic environments (gaseous or liquid ultracool nitrogen) to prevent the sample temperature from rising above the glass transition temperature $T_{\text {glass }}$, that is $T_{\text {glass }, H_{2} 0}=136 \mathrm{~K}$ [14] for water. Above this temperature the vitrified water matrix can begin to turn crystalline, resulting in cell damage and strong scattering contributions from the formed crystallites.

The mounting procedure for cryogenic samples is illustrated in Fig. 3.1 (b): The samples are transferred to a small liquid nitrogen bath for sample handling (typically a foam dewar), where the silicon nitride membranes are attached to a small goniometer base adapter with a specialized clamp. When the sample is in the clamp gap, the gap is tightly closed. A small vial connected to a vial tongue is cooled down in and filled with liquid nitrogen. Next, the goniometer base adapter with the sample attached is grabbed by a pair of strong self closing tweezers and positioned in the vial upside down. The sample is transported inside the vial to the sample adapter. The silicon nitride membrane is positioned exactly in the designated sample position of the cryostream, while still surrounded by the liquid nitrogen of the vial. Then the vial is pulled away quickly, the cooling is now guaranteed by the cryostream. To connect the (ferro-magnetic)

15 This procedure is recomended by the manufacturer. 
goniometer base adapter to the magnetic base plate of the sample stage, it has to be rotated around the silicon nitride membrane until the goniometer base is safely attached to the base plate.

The last two steps are very important for the success and require significant practice from experimentalists. Before the experiment is conducted, the transfer should be practiced repeatedly, in order to develop a procedure that takes into account personal (e.g., left/right handedness) and experimental (like the accessibility of sample support) conditions.

Unmounting without heating the sample is in principle possible by following the mounting procedure in the reversed order, i. e., turning the sample carefully upside down while the membrane stays in the cryostream at all times, approaching a vial with liquid nitrogen from below until the sample is immersed in liquid nitrogen and transporting the sample inside the vial back to a dewar. Still, this protocol is even more challenging and risky than the mounting procedure and many samples got lost this way. Even some that seemed to have been transferred without air contact showed ice crystals at the successive experiment.

The following modifications could improve the handling of the sample significantly:

1. If the magnetic base plate held the goniometer base from the top instead from below, the critical step of turning the sample freely in the cryostream would be avoided. This is even more relevant for unmounting the sample which would become as easy as mounting.

2. The second source of sample loss is the handling in a liquid nitrogen bath which is complicated due to the constant boiling of liquid nitrogen. A solution is to evacuate the liquid nitrogen bath for a short while [70]. The liquid is cooled further down by evaporation chill and the boiling stops under ambient pressure, until it heats up again. The resulting cooled liquid nitrogen bath shows no evaporation bubbles and thus no impact of bubbles onto the sample, and more important, a perfectly calm liquid surface with a clear view onto the preparation process.

\subsubsection{Microfluidic Chambers for Living Cells:}

Living cells were measured in x-ray compatible microfluidic devices (ibidi GmbH, Munich, Germany). The channel is defined by a $5 \mathrm{~mm}$ wide and $50 \mathrm{~mm}$ long slit in the spacer. Silicon nitride windows are fitted into the channel floor and top to reduce background scattering. A sketch and photograph of the chamber taken during experiments is shown in Fig. 3.1 (d), with the chamber outlined in red. 


\subsection{X-ray Fluorescence Measurements}

X-ray fluorescence aquisitions are possible simoultaneously to scanning SAXS, as, in principal, only a fluorescence detector needs to be installed and can record the fluorescence signal without significant interference with the scattering experiments.

We installed an AXAS-M fluorescence detector ${ }^{16}$ with an AXAS-M preamplifier and Xia Mercury multi channel analyser ${ }^{17}$ to analsze the signals. The data were processed in a dedicated instance of Epics ("Experimental Physics and Industrial Control System", control software developed by Argonne National Laboratory, Argonne, IL, USA). The data are transferred to the beamline control software Spec, which finally saves and displays the information ${ }^{18}$.

The detector was installed at an angle of $90^{\circ}$ with respect to the primary beam, situated in the positive $\mathrm{y}$-direction relative to the sample. However, the distance to the sample is not known precisely, it ranged between $3 \mathrm{~cm} . .30 \mathrm{~cm}$. X-ray fluorescence measurements allow to quantitatively measure the concentration of elements within a sample. By scanning the sample with respect to the focus position, the intensity $I_{f l}\left(E_{f l}, y, z\right)$ of the fluorescent light is recorded as a function of the lateral position $(y, z)$ and the energy of the photons $E_{f l}$.

The fluorescence spectra contain 4096 intensity values, which correspond to events per energy range, so-called channels. A calibration needs to be done by assigning peaks in this spectrum to emission lines of elements, that are known to occur in the dataset. The corresponding energy is then calculated from the channel number $N_{\text {channel }}$ using a second order polynomial of the form

$$
E_{f l}=A_{f l}+B_{f l} N_{\text {channel }}+C_{f l} N_{\text {channel }}^{2}
$$

with three calibration parameters $A_{f l}, B_{f l}$, and $C_{f l}$.

The software PyMCA [71] offers handling of data readily available including the fitting of data, peak identification tools, energy calibration, and element mappings of raster scans. Until here, only the peak fitting and calibration tools have been used, but for future applications PyMCA provides a batch fitting routine that includes many effects of fluorescence measurements (background models, pile-up peaks, and fitting of element spectra to the data).

During a beamtime, the fluorescence spectra changed so that the peaks in the spectra broadened and finally split, i.e., every peak was doubled. The manufacturer found a

16 by Ketek GmbH, München, Germany

17 Xia LLC, Hayward, CA, USA

18 Implementation by S. Kalbfleisch. 
cable damage that caused an error in the electronics of the detector system, which caused these ill-shaped results. All fluorescence data were measured before the repair. Fig. A.1 shows the effect of the defect, which results in the doubling of the peaks.

As a consequence, many spectra could not be considered for evaluation, but some early datasets could be analysed.

\subsection{X-ray Diffraction Setups}

The x-ray diffraction experiments for this thesis were done at three beamlines, the P10 (PetraIII, DESY, Hamburg), ID13 (ESRF, Grenoble), and cSAXS (SLS, Villigen, Switzerland).

\subsubsection{GINIX/P10 at PetralII}

This section 3.6.1 is an excerpt from the publication $[16]^{19}$.

X-ray measurements were performed at the Göttingen Instrument for Nano-Imaging with X-rays (GINIX) [72-74], at the P10 beamline of the synchrotron radiation source PETRAIII (Desy Photon Science, Hamburg), see also Fig. 2.3 (a). The undulator was operated in the first harmonic. The beam was monochromatized by a $\mathrm{Si}(111)$ double crystal monochromator to a photon energy $E_{\lambda}=7.9 \mathrm{keV}$, and focused by a pair of elliptically shaped Kirkpatrick-Baez $(\mathrm{KB})$ mirrors to $326 \mathrm{~nm}(h) \times 392 \mathrm{~nm}(v)(\mathrm{FWHM})$, with a total photon flux of $I_{0}=1.1 \cdot 10^{11} \mathrm{ph} . / \mathrm{s}$, as measured with the photon counting pixel detector Pilatus 300K (Dectris Ltd, Baden, Switzerland), positioned 5.29m behind the sample, which is placed in the focal plane of the KB. The beam is partially coherent with a global degree of coherence 0.36 and 0.68 , in the horizontal and vertical direction, respectively [73]. The beam can be made fully coherent by closing the slits in front of the KB, at the expense of lower flux and a focal spot size which broadens by diffraction. Depending on the exact alignment and ring orbit, spot sizes below $200 \mathrm{~nm}$ can also be achieved. Of particular importance for the present nano-focus diffraction measurements is the soft-edge tantalum aperture [75] which is placed $3 \mathrm{~mm}$ upstream from the focal plane to reduce the background scattering. A tantalum aperture was used to suppress the pronounced streaks of typical KB far-fields, which can contaminate the diffraction patterns of the sample. This aperture with soft edges successfully reduces the background, see the far-field pattern of the empty beam, presented in Fig. 5.1 (b). Importantly, the pronounced streaks of typical KB far-fields are eliminated, which can contaminate the

19 M. Priebe, M. Bernhardt, C. Blum, M. Tarantola, E. Bodenschatz, and T. Salditt: Scanning X-Ray Nanodiffraction on Dictyostelium discoideum. Biophysical Journal, 107(11):2662-2673, 2014. 
diffraction patterns of the sample. As sketched in Fig. 2.3 (a) two motorized semitransparent beamstops (BS) [76] installed in the detector evacuated flight tube are employed to attenuate the direct (primary) beam. A $25 \mu \mathrm{m}$ thick tungsten plate of $8.0 \mathrm{~mm} \times 7.0 \mathrm{~mm}$ covers the primary beam and a larger silicon plate of $280 \mu \mathrm{m}$ thickness measuring $17.1 \mathrm{~mm} \times 12.0 \mathrm{~mm}$ attenuates a larger area around the central beam. The effect of the two beamstops is readily inspected in the far-field pattern shown in Fig. 3.2.

A $x y z$ piezo stage P-615 NanoCube ${ }^{20}$ with a travel range of $(x \times y \times z) 350 \mu \mathrm{m} \times$ $350 \mu \mathrm{m} \times 250 \mu \mathrm{m}$ was used to scan the samples in the beam. Scanning can be performed stepwise through the beam. Typical step sizes were $250 \mathrm{~nm}$ to $2 \mu \mathrm{m}$, depending on the scan, typical dwell times were between $0.05 \mathrm{~s}$ and $0.5 \mathrm{~s}$. At each position a full diffraction pattern is recorded. Step sizes can be set separately for the columns and lines. For fast scanning which is particularly important for radiation sensitive samples, a continuous scanning mode is used. Here, linewise scanning is perfomed so that the motion is continuous along the lines. The detector readout is triggered according to the desired resolution along the line. The later mode resembles the normal scanning mode with an effectively broadened focus and overlap of one beamwidth. An inline optical microscope, (Maatel), positioned directly behind the sample, is used to identify the region of interest (ROI) on the sample and position it with respect to the beam, see Fig. 3.1 (e). To protect the sample from radiation damage, a $100 \mathrm{~K}$ environment is provided by a $N_{2}$ cryostream ${ }^{21}$, as sketched in Fig. 2.3 (a). A fast shutter triggered to the detector also helps to prevent unnecessary radiation exposure. Sample holders with magnetic bases and transfer vials are used to safely position the samples in the beam.

\subsubsection{ID13 at the European Synchrotron Radiation Facility}

Experiments were carried out at the nanobeam extension of the undulator beamline ID13. The radiation is monochromatized to a photon energy of $14.9 \mathrm{keV}$ by a $\mathrm{Si}(111)$ channel-cut monochromator. The beam was focussed with a set of micro compound refractive lenses $[77,78]$ to a focus diameter of approximately $100 \mathrm{~nm}$ (FWHM) with a primary intensity of $I_{0}=6.5 \cdot 10^{8} \frac{\mathrm{ph}}{\mathrm{s}}$, as determined with the Maxipix detector, which is installed at a distance of $L=2.86 \mathrm{~m}$ behind the sample position. An electron microscopy aperture of $20 \mu \mathrm{m}$ diameter is placed between the lens set and sample position to clean up the beam. A hexapod allows a rough positioning of the sample, on top of the hexapod is a piezo actuator for scanning purposes. Samples are attached to the top plate of the piezo stage by a strong magnet. A thin lead wire with a diameter of $d_{P b} \approx 100 \mu \mathrm{m}$ is

$\overline{20}$ by Physik Instrumente (PI) GmbH \& Co. KG, Karlsruhe, Germany

21 Oxford Cryosystems Ltd, Oxford, United Kingdom 
positioned a few $\mathrm{cm}$ behind the sample, held by a quartz glass capillary that it is attached to. The short distance between sample and beamstop has the advantage of very little air scattering, a flight tube is thus not employed in the nanofocus hutch.

The used Maxipix detector consists of $2 \times 2$ modules with overall $516 \times 516$ pixels, each pixel sized $55 \mu m \times 55 \mu m, 68 \%$ efficiency at $15 \mathrm{keV}$ (see ID13 homepage)) [79].

\subsection{3. cSAXS at the Swiss Light source}

The coherent Small-Angle X-ray Scattering (cSAXS) beamline is an undulator beamline dedicated for coherent scattering, operated by the Swiss Light Source (SLS) at the Paul Scherrer Institut (5232 Villigen PSI, Switzerland). One focus of the beamline are coherent imaging techniques like ptychography, but scanning SAXS is possible as well. We applied both techniques to frozen-hydrated and freeze-dried samples of $D$. discoideum and Deinococcus radiodurans (D. radiodurans).

The beamline includes an undulator source followed by slits, a liquid nitrogen cooled $\mathrm{Si}(111)$ double crystal monochromator, and a set of attenuation filters before the focussing optic. A fresnel zone plate (FZP) is used for focussing. The FZP is made from gold $\mathrm{Au}$ ) on a silicon nitride membrane by e-beam lithography, with a zone height of $1 \mu \mathrm{m}$. The diameter measures $200 \mu \mathrm{m}$, the outer zone width is $d r=100 \mathrm{~nm}$. The radiation transmitted through the FZP interferes constructively in a small focal spot at the drawback of several maxima. These higher diffraction orders result in several foci with shorter focal distance from the FZP and more than one imprint of the primary beam on the detector. An order sorting aperture with a diameter of $20 \mu \mathrm{m}$ is used to suppress the higher orders. It is placed between the FZP and the focal position. The central stop $(40 \mu \mathrm{m})$ is positioned in front of the FZP. The optics achieves a diameter in the focal spot of $600 \mathrm{~nm}$ (FWHM, as determined from a ptychographic reconstruction of the probe).

The scattered radiation is guided through a $7 \mathrm{~m}$ long helium filled flight tube to reduce air scattering. A Pilatus 2M detector (Dectris Ltd, Baden, Switzerland) is installed at a distance of $7.63 \mathrm{~m}$ from the focus/sample position, the detector offers an active area of $1475 \times 1679$ pixels, which have a dimension of $172 \mu m \times 172 \mu m$ each. Details on the setup and our experiment can be found in [80].

The sample translation stage involves from bottom to top: a hexapod (Physik Instrumente (PI) GmbH \& Co. KG, Karlsruhe) for rough positioning, an air bearing rotation (Micos GmbH, Eschbach, Germany) around the vertical axis ( $y$ in cSAXS coordinate system), two linear translation stages in the $x$ - $z$-plane, and a linear piezo stage (PI) with three axis for scanning fast scanning purposes. The linear translations above the rotation are for sample alignment, the piezo stage allows fast and accurate positioning, but is 
not suitable for alignment of the sample as the travel range is smaller than the typical membrane size. A movement of the hexapod below the rotation allows the positioning of the rotation axis with respect to the beam. Tomographic measurements of single cells is thus enabled. Experimental setup and applied aquisition and reconstruction schemes are detailed in [80].

We worked at $E_{\lambda}=6.2 \mathrm{keV}$ with an intensity of $I_{\text {coh. }} \approx 7 \times 10^{8} \mathrm{ph} / \mathrm{s}$ for the coherent and $I_{\text {incoh. }} \approx 1 \times 10^{10} \mathrm{ph} / \mathrm{s}$ for the incoherent slit setting (as measured with the Pilatus detector). The higher degree of coherence required for Ptychography is achieved by closing of the slits $S 0$ close to the source to a width of $d_{S 0, c o h}=100 \mu \mathrm{m}$. For diffraction aquisitions, the slits could be opened further to $d_{S 0, \text { incoh. }}=420 \mu \mathrm{m}$ in order to increase the flux. 


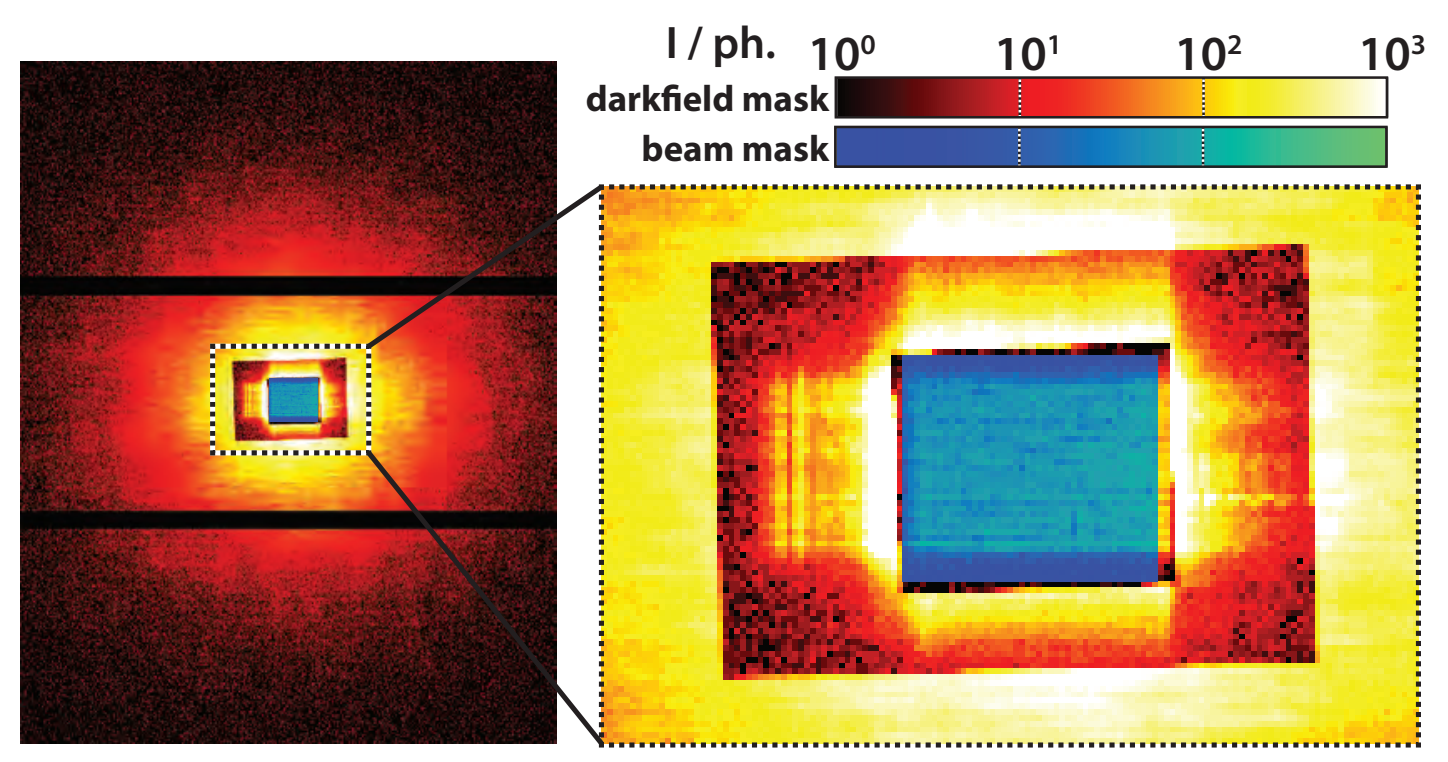

Figure 3.2.: An exemplary diffraction pattern is shown here to illustrate the masks that are applied in the STXM contrasts. The darkfield mask is displayed in a red color scheme and the beam mask, which is defined as the inverse of the darkfield mask, is represented in a blue to green color code. The darkfield mask covers the whole detector image exept for the area of the primary beam, as becomes clearer in the inset. The challenge in the choice of masks is that the central beam is very close to the right side of the inner beamstop and thus cannot be fully framed without considering signal that passed through different absorbers. In this case the different intensity levels would cause an unrealistically high contrast. Material of this figure and caption was also published in [16]. 


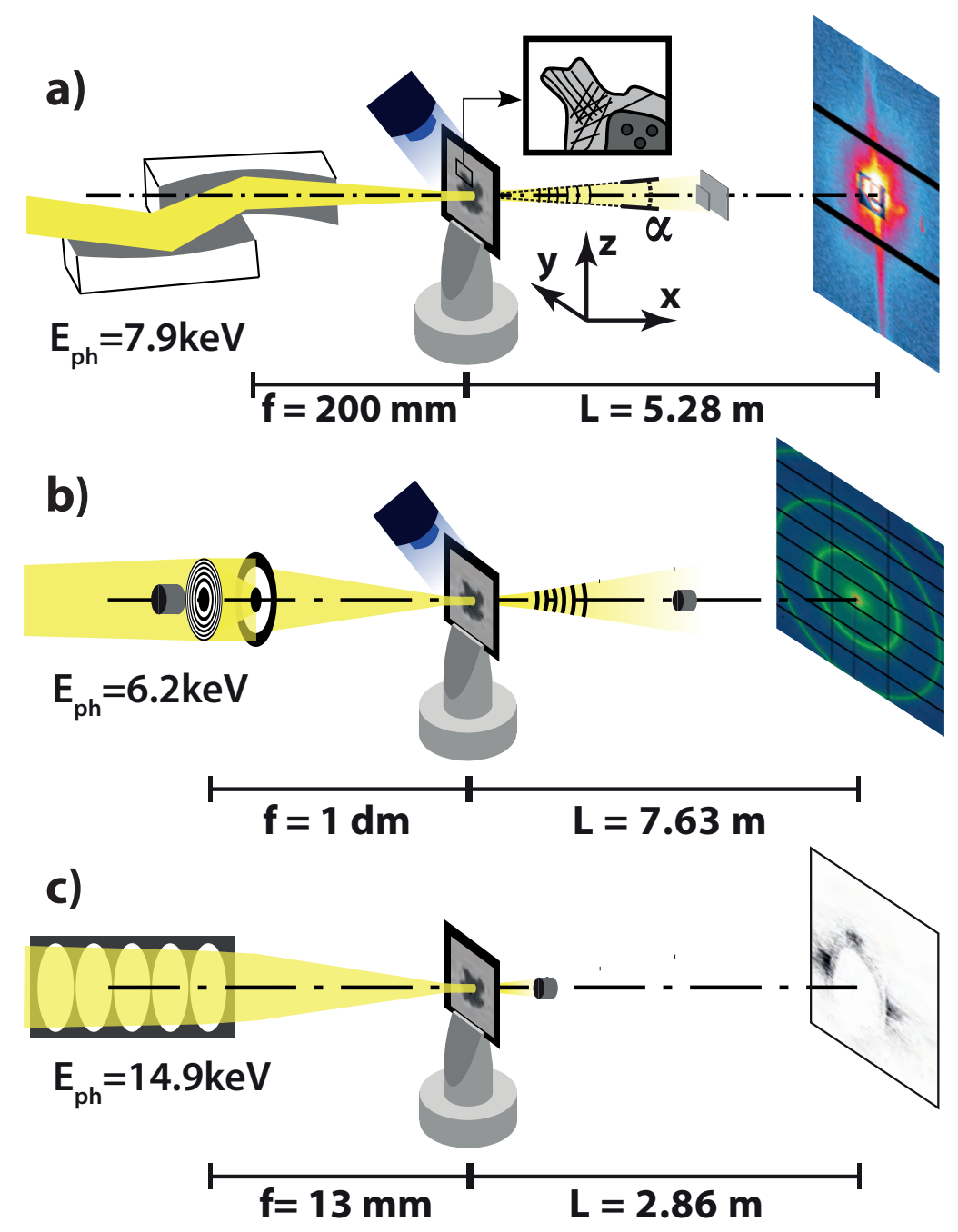

Figure 3.3.: Sketches of the x-ray diffractometers at P10, cSAXS and ID13. (a) Sketch of SAXS setup used at the GINIX with (1) a pair of Kirkpatrick-Baez-mirrors, (2) the cryostream directed onto (3) the sample on the goniometer base positioned at the focal distance $f=200 \mathrm{~mm}$ from the second mirror, (4) the semitransparent beamstops in the flight tube (not shown) and (5) the Pilatus 300K detector at the distance $L=5.28 \mathrm{~m}$. (b) cSAXS setup used for nano-diffraction and Ptychography: The incoming radiation is focussed by (7) a Fresnel zone plate (FZP) with preceeding (6) central beam stop and (8) an order sorting aperture. (9) The sample is placed in the focus of the FZP and cooled by (10) a cryostream. (11) A beamstop inside the flight tube (not shown) is employed for the nano-diffraction experiments, but left out when recording ptychographic aqcuisitions. The radiation is recorded with (12) a Pilatus pixel detector. (c) At the diffraction setup in the third hutch of ID13, a (13) set of nano compound refractive lenses (CRLs) focusses the incoming radiation. (14) The sample is placed at the focal distance of $13 \mathrm{~mm}$ and the primary beam is blocked directly behind the sample by a specialized beam stop, that consists of a small lead wire glued to a glass capillary. The scattered radiation is recorded by a $2 \times 2$ element Maxipix pixel detector . Material of this figure and caption was also published in [16]. 


\section{The Streak Finder}

In this chapter a novel anisotropy parameter $\check{S}$ is introduced, which allows to characterize the anisotropy of a diffraction pattern by a single number. It may thus serve as a contrast of STXM recordings. The anisotropy parameter maps can be complemented with a small indicator bar that represents the main axis of the intensity distribution in the far-field.

This chapter begins by giving a definition of the anisotropy parameter and algorithm (nicknamed "Streak Finder") as it is presented in our paper [16]. Next, an additional normalization step is suggested, resulting in a well normalized anisotropy parameter $S$ which fulfills $0 \leq S \leq 1$. Test data have been generated to characterize the behaviour of the algorithm. A comparison to anisotropy parameter definitions introduced by Bunk et al. [81] and Weinhausen [11] is drawn before the chapter closes with briefly discussing possible upgrades of the algorithm.

\subsection{Algorithm}

The input of this algorithm is a single diffraction pattern from which a single anisotropy characterizing number is calculated. The coordinates of the beam centre are needed as one parameter.

First, the diffraction pattern is transformed from cartesian coordinates $q_{y}, q_{z}$ to polar coordinates $q_{r}, \chi$, which facilitates the following renormalization and integrations along $q_{r}$ and $\chi$. The intensity on the detector decreases along $q_{r}$ by typically two or more orders of magnitude, so that long streaks may extend over the whole detector, but the relative contribution of the signal at large $q_{r}$ to the azimuthal intensity profile $\left\langle I\left(q_{r}, \chi\right)\right\rangle_{q_{r}}$ is small. Therefore, the average of the intensity distribution over the azimuthal angle

$$
\left\langle I\left(q_{r}, \chi\right)\right\rangle_{\chi}=\frac{1}{2 \pi} \int_{0}^{2 \pi} I\left(q_{r}, \chi\right) \mathrm{d} \chi
$$

is used to normalize the intensity distribution, resulting in the weighted intensity distribution

$$
I_{w}\left(q_{r}, \chi\right)=\frac{I\left(q_{r}, \chi\right)}{\left\langle I\left(q_{r}, \chi\right)\right\rangle_{\chi}} .
$$


The average $\left\langle I\left(q_{r}, \chi\right)\right\rangle_{\chi}$ acts here as a mutual intensity decay profile, which we employ for a radial weighting.

The relative intensity profile along $\chi$ is obtained by integration along $q_{r}$ of the weighted intensity

$$
I_{r e l}(\chi)=\int_{q_{\min }}^{q_{\max }} I_{w}\left(q_{r}, \chi\right) \mathrm{d} q_{r}
$$

Next, the discrete Fourier tranform is applied to $I_{r e l}$

$$
\tilde{I}(k):=\sum_{n_{\chi}=0}^{N-1} I_{r e l}\left(n_{\chi}\right) \cdot \exp \left(\frac{-2 \pi \imath}{N} \cdot k \cdot n_{\chi}\right), 0 \leq k \leq N-1,
$$

with $n_{\chi}$ beeing the discrete angular variable that replaces $\chi . \tilde{I}(k)$ is used to define the anisotropy parameter $\check{S}$

$$
\check{S}:=\frac{1}{\|\tilde{I}(0)\|} \sum_{k=1}^{6}\|\tilde{I}(2 k)\| .
$$

The decision to consider 6 even components for the summation was made during the first construction of the steak finder algorithm and it proved to work well for the datasets considered in this work. The role of this number is discussed later in this chapter.

The implementation of this definition is illustrated in Fig. 4.1. Starting from (a) an anisotropic diffraction pattern, (b) the coordinate transformation is plotted with two hatched regions, which are not considered for the following steps. The area of small $q_{r} \leq q_{r, \text { min }}$, where the shadow of the beamstop would cause anisotropic signal is left out. So is the region of large $q_{r} \geq q_{r, \max }$, where only the corners tips of the detector would contribute. The weighted intensity distribution $I_{w}\left(q_{r}, \chi\right)$ in (c) differs from the unweighted one by a relatively constant intensity along the streaks and an increase of the background signal along $q_{r}$. The variation of the number of active pixels per line (i. e., at a given $q_{r}=$ const.) is accounted for in the averaging process. Summation along $q_{r}$ yields (d) the azimuthal profile, in red the example from (a-c), in blue a curve that was obtained in the same way as before, but with the isotropic scattering signal from the spot on a cell. (e) The FFT of the data shown in (d) finally allows to discriminate the isotropic from the anisotropic scattering patterns.

A few artefacts can be seen in (c), where the weighting of $I\left(q_{r}, n_{\chi}\right)$ leads to regions of lower or higher intensities in the background. The reason lies in the definition of the arithmetic mean

$$
\left\langle I\left(q_{r}, n_{\chi}\right)\right\rangle_{n_{\chi}}=\frac{1}{\# \text { activepixels }} \sum_{\text {activepixels }} I\left(q_{r}, n_{\chi}\right),
$$


which is used for the normalization along $q_{r}$. Pixels, that are outside the detector region or on intermodule gaps ${ }^{1}$ are not considered for averaging. If these pixels are in a streak region, the number of pixels with high intensities is relatively smaller resulting in a smaller value for $\left\langle I\left(q_{r}, n_{\chi}\right)\right\rangle_{n_{\chi}}$. This leads to larger values in the reweighted intensity distribution $I_{w}\left(q_{r}, n_{c} h i\right)$. The argument applies for insensitive pixels in areas between streaks accordingly. As these lower or higher intensities occur only within a small part of the $\left[q_{r, \min }, q_{r, \max }\right]$ range, the artefact has little contribution to the azimuthal profile.

In addition, the orientation of the streak is important structural information. It is determined by smoothing the $I_{\text {rel }}(\chi)$ curve with a box filter of size five (see lines 81 and 197 in the Streak Finder algorithm, section C.0.1), and taking the position of the maximum.

The information of the orientation of the streaks is also contained in the phase of the Fourier components. For a symmetric peak, the phase of the first even Fourier component should correspond to the orientation of the streak. It could thus serve as an orientation parameter. However, a test on actual data revealed that this approach is less accurate than the determination of the maximum of the azimuthal profile.

This definition is an empirical finding based on the observations made during data analysis. It makes use of the point symmetry in diffraction patterns, and suppresses contributions from non point symmetric intensity values. As it turned out, the anisotropy parameter does not only nicely point out highly oriented diffraction patterns, but as far as can be judged by the eye, it provides a reliable measure for the amount of orientation in typical diffraction patterns. First of all, this means that streaky diffraction patterns result in high values between $0.5 \leq \check{S} \leq 2$, depending on the intensity and the sharpness of orientation. But also relatively isotropic diffraction patterns can show very small degrees of anisotropy (e.g., the empty beam image sometimes shows a slightly larger $\breve{S}$ than the scattering signal of a cell, as beam artefacts can cause orientation in the background), which means that it "resolves" the anisotropy observed with the eye more than satisfactorily.

In the presented definition of the Streak Finder, the sum over the Fourier components is normalized with the absolute of the zeroth Fourier component, i. e., the total intensity of the relative intensity profile $I_{r e l}$. As this profile originates from an already weighted intensity map, this second normalization technically only compensates for artefacts from the first weighting. In theory this should not be necessary, but the intermodule gaps cause the absolute intensity to be relatively higher or lower (around ten percent in total).

$1 \quad$ The Pilatus detector is built from modules (three for the Pilatus 300k) that are arranged with a small gap between adjacent modules, the so-called intermodule gap. The detector is not sensitive to photons there. In diffraction pattern these gaps are visible as two horizontal stripes. 

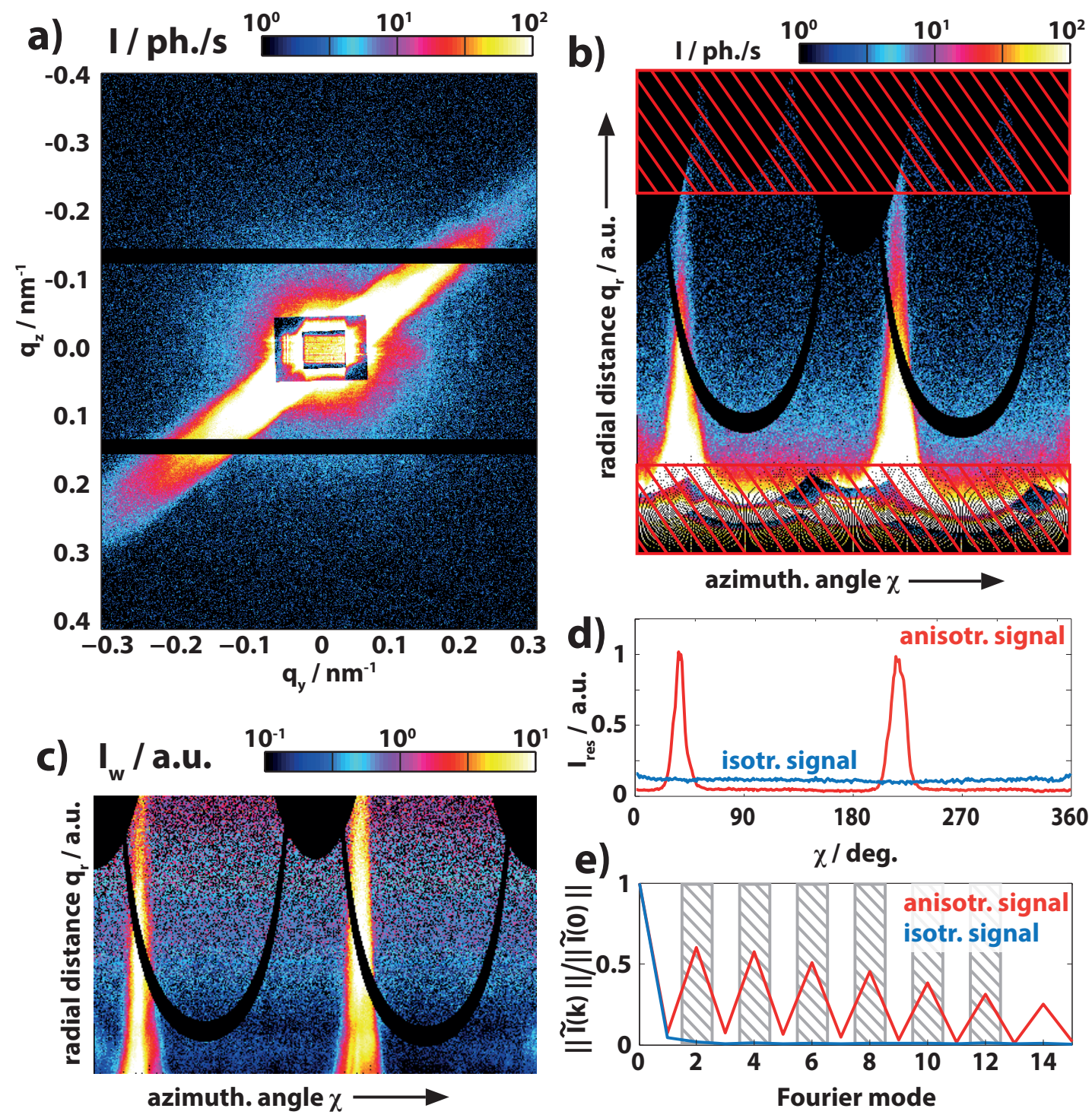

Figure 4.1.: Schematic of the Streak Finder algorithm starting with (a) the original diffraction pattern, which is transformed to (b) cartesian coordinates $I\left(q_{r}, \chi\right)$. Regions of small and large $q_{r}$ are omitted (hatched) and the image is radially reweighted to emphasize the streak signal (c). Integration along the former radial axis $q_{r}$ yields (d) an azimuthal profile that is expanded into (e) its normalized Fourier modes $\tilde{I}(k) / \tilde{I}(0), k \in[0,15] .(\mathrm{d}+\mathrm{e})$ show the distributions of the ansiotropic diffraction pattern shown in (a-c) in red and the results of yet another (isotropic) diffraction pattern measured on a cell in blue for comparison. Material of this figure and caption was also published in [16]. 
This artefact occurs mainly at streak positions and is strongly biased with the orientation of the streaks, which makes the weighting necessary.

\subsection{Normalization}

The anisotropy parameter is conceptually designed as an order parameter. It should reach a maximum value for a 'single orientation' (a definition will be addressed below) and 0 for fully isotropic scattering. The Fourier transform accurately captures isotropic scattering patterns, as for this case only the zeroth order component is nonzero, resulting in $\breve{S}=0$. But how could the "strongest possible orientation" look like? In theory, it would be represented by a diffraction pattern with two delta peaks, that point out radially and appear point symmetric around the optical axis of the primary beam. The background is assumed to be zero for now. One can then calculate the anisotropy parameter with the Streak Finder algorithm of this model function $t(n)$. Using (discrete) delta distributions

$$
\delta(n):=\left\{\begin{array}{ll}
1 & \text { if } n=0 \\
0 & \text { else }
\end{array} .\right.
$$

to define sharp maxima results in a test function $I_{t}(n)$

$$
I_{t}(n):=A \delta\left(n-n_{s}\right)+A \delta\left(n-\left(n_{s}+\frac{N}{2}\right)\right)
$$

with an amplitude $A>0$ and streak direction $n_{s} \in[0, N-1]$. Point symmetry is only given for even numbers of $N$. We use the following definition of the discrete Fourier transform

$$
\tilde{I}(k):=\sum_{n=0}^{N-1} I(n) \cdot \exp \left(\frac{2 \pi}{N} k n\right)
$$

and apply it to our model intensity distribution

$$
\begin{aligned}
\tilde{I}_{t}(k) & =\sum_{n=0}^{N-1}\left[A \delta\left(n-n_{s}\right)+A \delta\left(n-\left(n_{s}+\frac{N}{2}\right)\right)\right] \cdot \exp \left(\frac{2 \pi}{N} k n\right) \\
& =A \exp \left(\frac{2 \pi}{N} k n_{s}\right)+\exp \left(\frac{2 \pi}{N} k\left(n_{s}+\frac{N}{2}\right)\right) .
\end{aligned}
$$


The value of $n_{s}$ sets the position of the streaks and depends on the choice of coordinate system. As only the absolute of the Fourier componenents is considered, we may assume without loss of generality, that $n_{s}=0$, which results in

$$
\begin{aligned}
\tilde{I}_{t}(k) & =A(1+\exp (\pi k)) \\
& =\left\{\begin{array}{rl}
0 & \text { for odd } k \\
2 A & \text { for even } k
\end{array} .\right.
\end{aligned}
$$

The resulting value of $\check{S}$ is

$$
\begin{aligned}
\check{S} & =\frac{1}{\left\|\tilde{I}_{t}(0)\right\|} \sum_{k=1}^{6}\left\|\tilde{I}_{t}(2 k)\right\| \\
& =\frac{1}{2 A} \cdot 6 \cdot 2 A \\
& =6
\end{aligned}
$$

By taking into account, that intensity values are always real and non-negative, the definition of the Fourier transform implies, that $\tilde{I}_{f}(0) \geq \tilde{I}_{f}(n)$. This implies for a renormalized Streak Finder, that dividing $\breve{S}$ by the number $N_{S F}$ of considered Fourier modes (here $N_{S F}=6$ ) results in a renormalized anisotropy paramter $S$ which fulfills $0 \leq S \leq 1$

$$
S:=\frac{1}{\left\|\tilde{I}_{t}(0)\right\|} \frac{1}{6} \sum_{k=1}^{6}\left\|\tilde{I}_{t}(2 k)\right\| .
$$

Until here, $N_{S F}=6$ was chosen, but this is not necessarily the only possible choice. $N_{S F}$ allows to adapt for different signal shapes:

$$
S_{N_{S F}}:=\frac{1}{\left\|\tilde{I}_{t}(0)\right\|} \frac{1}{N_{S F}} \sum_{k=1}^{N_{S F}}\left\|\tilde{I}_{t}(2 k)\right\| .
$$

Smaller $N_{S F}$ can account for broader peaks and higher numbers of $N_{S F}$ allow a more accurate sampling of peaks with a small full width at half maximum.

\subsection{Characterization of the Streak Finder}

In this section, regions of insensitive pixels in the Streak Finder ROI will play a major role, therefore the following nomenclature is introduced: If $q_{r, \max }$ exceeds the detector 
size, empty regions will occur in the ROI, this case shall be called rectangular detector. Otherwise, round detector will be used, see also Fig. 4.2. Test data were generated to test and characterize the Streak Finder. The constructed intensity distribution has the form of a two dimensional Gaussian distribution

$$
I_{\text {test }}(x, y)=\frac{A}{2 \pi \sigma_{\|} \sigma_{\perp}} \cdot \exp \left(-\frac{1}{2}\left(\frac{x-\mu_{\|}}{\sigma_{\|}}\right)^{2}\right) \cdot \exp \left(-\frac{1}{2}\left(\frac{y-\mu_{\perp}}{\sigma_{\perp}}\right)^{2}\right)+B
$$

where $\sigma$ denotes the distribution width, $\mu$ the centre of the distributions and $A$ an amplitude to the distribution function. The constant $B$ allows to add a constant background. The two directions are indexed for the components in parallel and perpendicular direction with respect to the main orientation of the streak. The data are first generated and then rotated with the matlab function imrotate to match the desired orientation angle. A mask can be applied to simulate dead regions in the data as found in diffraction images from the Pilatus detector. The empty circle around the

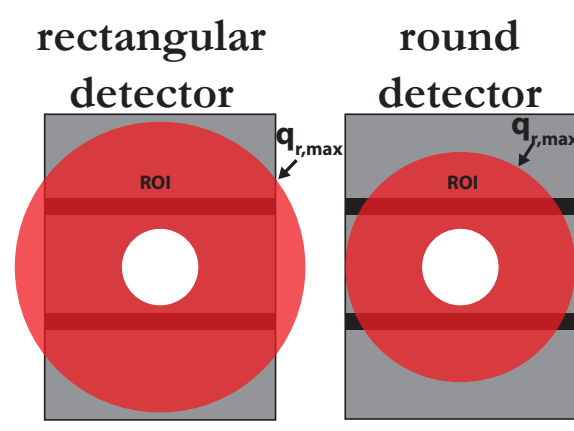

Figure 4.2.: On the definition of nomenclature for ROIs larger or smaller than the detector. beam centre corresponds to the inner radius of the ROI that is considered.

Optimal conditions A first test was made without the intermodule gaps of the Pilatus detector or dead regions from the sides. The transformed and reweighted intensity distribution is shown in Fig. 4.3 a). The streak orientation was varied and the resulting anisotropy parameter and streak angle plotted vs. the simulated streak orientation. The angle was determined accurately (within the discretisation of the sampling rate of $\chi$, given by the number $N$ of angular segments, here $N=360$ ) and the anisotropy parameter shows variations on the order of $10^{-3}$ of its value. This proves the correct functioning of the algorithm. The slight variation could be due to few empty pixels, that result from the coordinate transformation at low $q_{r}$ or numerical residues. Secondly, the contributions of the areas without intensity information c) resulting from the sides of the detector are simulated, the output of the Streak Finder algorithm is presented in d). The resulting shape of the anisotropy parameter curve is found to be characteristic for strongly anisotropic intensity distributions with a decay along $q$ (see following paragraph). 
a) reweighted intensity distributions

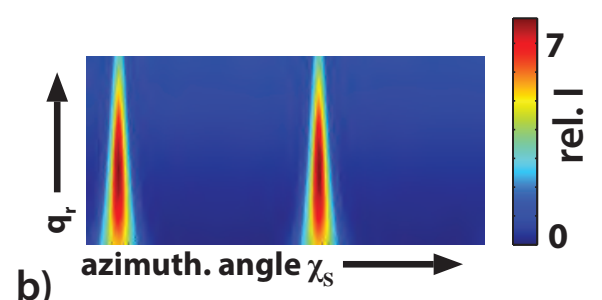

b)

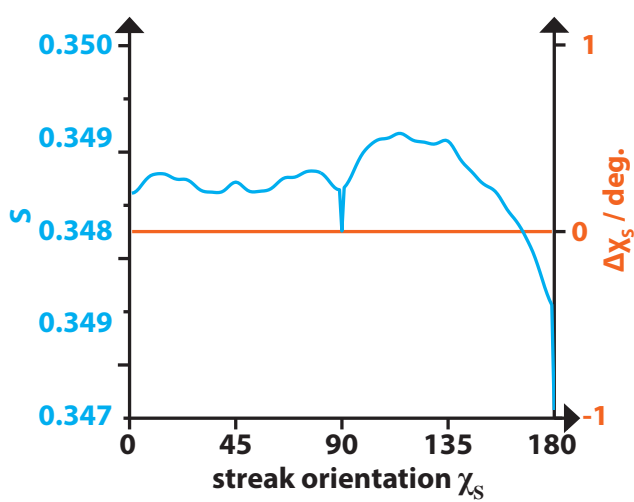

c)

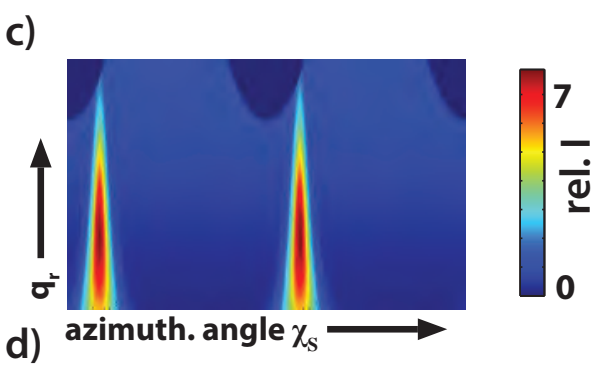

d)

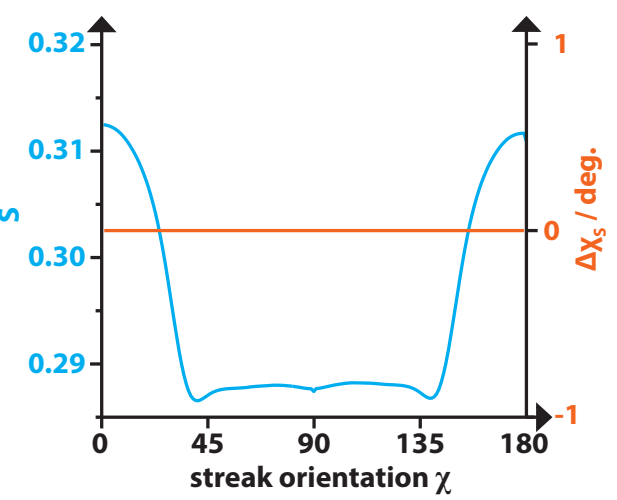

Figure 4.3.: Test data without intermodule gaps from a) a round and c) a reactangular detector. The resulting anisotropy parameter $S$ and angular deviation $\Delta \chi_{S}$ are shown in b) for the round detector and d) for the rectangular one with respect to the streak orientation. The test data had a background of $B=2$, streak widths of $\sigma_{\|}=100$ and $\sigma_{\perp}=15$ and an intensity of $A=10^{6}$. 

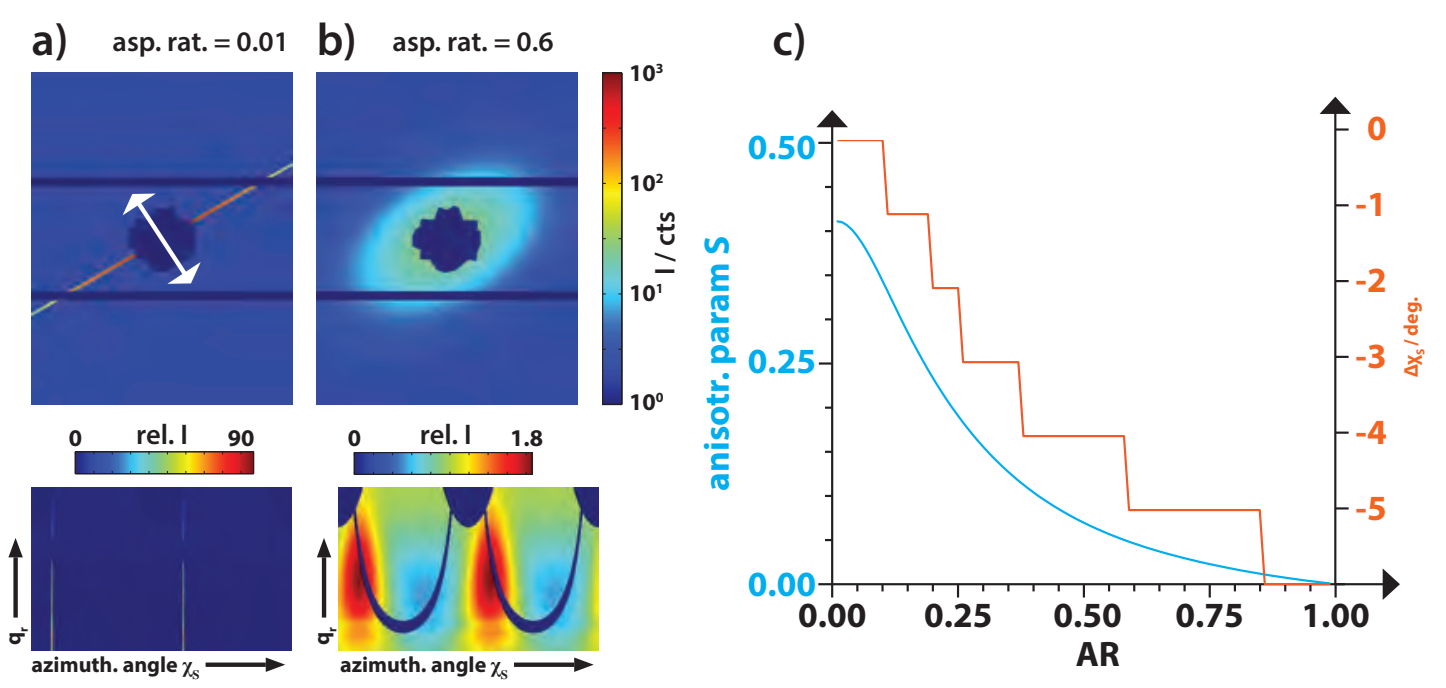

Figure 4.4.: Characterization of Streak Finder algorithm with respect to the aspect ratio $A R=\frac{\sigma_{\perp}}{\sigma_{\|}}$of the intensity distribution. Test data $(\mathbf{a}, \mathbf{b})$ with $\sigma_{\|}=100 p x$ and varying $\sigma_{\perp} \in[1 \ldots 99 p x]$ are processed in the Streak Finder algorithm, the resulting anisotropy parameter and deviation of the actual orientation are plotted as a function of the aspect ratio (c). The small images below a) and b) are the reweighted intensity distributions obtained from the data. The angle of the main axis was fixed at $\chi=30^{\circ}$, with a background $B=2$ and fixed streak intensity $A=10^{6}$.

Distribution width $\mathrm{A}$ distribution with $\sigma_{\|}=100 p x$ and varying $\sigma_{\perp} \in[1 p x, 99 p x]$ was processed with the Streak Finder algorithm. The background is set to $B=2$ and the amplitude to $A=10^{6}$. Fig. 4.4 (a+b) shows two of the resulting test datasets with the exemplary aspect ratios $A R=\frac{\sigma_{\perp}}{\sigma_{\|}}$of (a) $A R=0.01$ and (b) $A R=0.6$. All test data were generated with a fixed streak orientation of $\chi_{s}=30^{\circ}$. The resulting anisotropy parameter and angular deviation $\Delta \chi_{s}$ (i. e., the difference between the calculated angle and the actual angle defined in the test data) are shown in Fig. 4.4 (c). The anisotropy parameter decays in a slope from $S=0.42$ to $\approx 0$. The angle deviates from the chosen angle by no more than $\Delta \chi_{s} \leq 6^{\circ}$.

Orientation and background Fig. 4.5 (c-f) illustrate the behaviour of $S$ and $\Delta \chi_{s}$ with varying $\chi_{s}$ and for different background levels. An example of the intensity distribution put to test is shown in a) with the corresponding transformed and reweighted intensity distribution in b).

Compared with Fig. 4.3, the overal trend is retained, only the insensitive detector regions add some high frequency features. The anisotropy parameter graphs of different 
a)

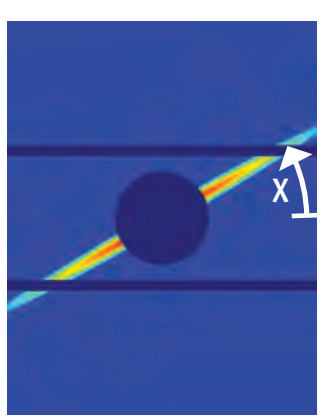

b) $0 \quad$ rel. I 20

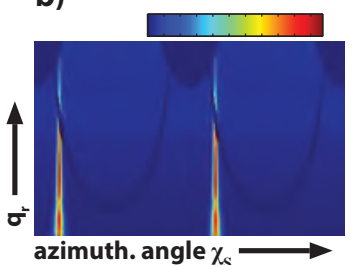

c) $B=2 c t s$

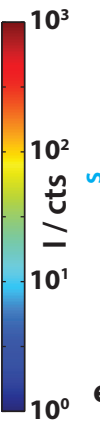

C) $B=2$ cts

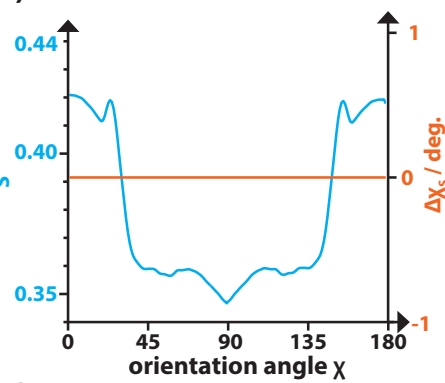

e) $B=200 \mathrm{cts}$

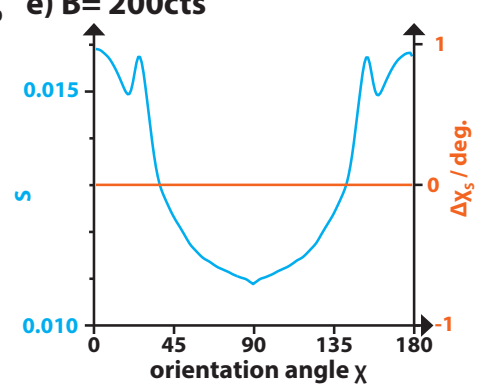

d) $B=20 \mathrm{cts}$

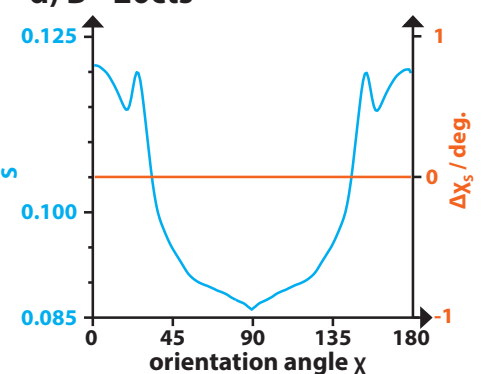

f) $\mathrm{B}=2000 \mathrm{cts}$

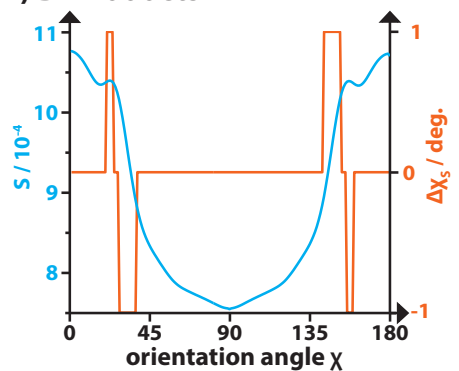

Figure 4.5.: Characterization of the Streak Finder algorithm for different background levels. Test distributions $\left(A=10^{6}, \sigma_{\|}=100, \sigma_{\perp}=5\right.$, a) and b) show the case for $\left.B=2\right)$ are fed into the Streak Finder algorithm with different background levels $B$. The resulting $S$ and $\Delta \chi_{S}$ are shown in c)-f).

a)
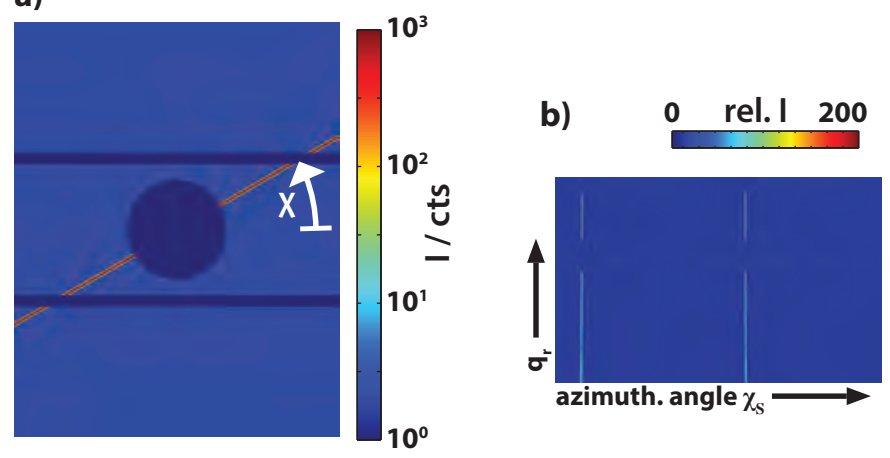

c)

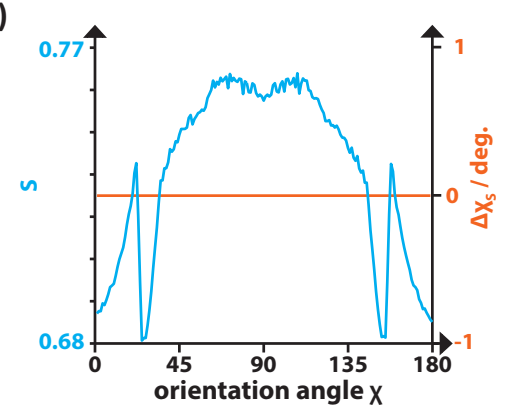

Figure 4.6.: A box shaped distribution that is 1000 inside and 0.01 outside the 5 px wide streak is tested and the result plotted in c). 
backgrounds resemble each other. Some local features are smeared out and systematic deviation of the angle $\Delta \chi_{S}$ appears in f). Interestingly the relative variation in $S$ is constant at roughly $\frac{\Delta S}{S} \approx 0.3$.

Fig. 4.6 (a) shows a box shaped intensity distribution, that was 1000 inside and 0.01 outside of the streak. The streak measured $5 p x$ in width. In contrast to the Gaussian streaks, the signal in the streak regions remains constant in $q$ direction. As a result, the anisotropy parameter shows the opposite trend as compared to the cases in Fig. 4.5. This also explains the origin of the variations: To determine $I(q)$, the reweighted intensity distribution is averaged along $q$. At angles close to $\chi=0^{\circ}$, the lowest intensities found at large $q$ are missing, so that the overall intensity at this angle is larger as compared to streaks at $\chi_{s}=90^{\circ}$, where also the lowest intensities are taken into account. When the signal is constant, this effect reverses, because the reweighting step along $q$ emphasizes the background when the streak signal is hidden.

\subsection{Possible Future Modifications}

High sensitivity for background An important conclusion of the simulations is the dependence of $S$ on the background level. If the background level is not negligible relatively to the signal intensity, a background subtraction must be performed to allow a comparison between measurements. All Streak Finder results in this work were obtained from raw data without background subtration. As the experimental conditions were nearly identical (same setup at GINIX) and large regions of the detector (mainly at high $q_{r}$ ) have very low background, the results shown should be comparable within this work.

Correction of anisotropy parameter In principal, the curves in Fig. 4.5 (c-f) allow to correct the anisotropy parameter for its angular derivations. However, different peak shapes and background levels limit this method; a certain degree of similarity in the streaks is required. An multi plot similar to Fig. 5.2 provides a good overview and could allow to taylor a test function (e.g., $I \propto q^{-3}$ and the width of the primary beam as seen on the detector) for an experiment.

Experiment specific calibration data can be derived from this test function and used as a lookup table to correct values obtained for different angular orientations. Artefacts from rectangular detectors can be avoided by choosing the $q_{r, \text { max }}$ smaller.

Round detectors and intermodule gaps The influence of intermodule gaps and rectangular detectors depends on the number of pixels in the ROI which is considered in the calculation algorithm. It should in principle be possible to extrapolate the unknown 
intensity in these areas. A simple approach could be to assume a linear shape for the unknown signal in gaps along $q$ direction. Areas without information that lie on one side of the ROI would need an assumption or model for the signal shape. Fig. 5.2 suggests $I \propto q^{-3}$, that would need to be fitted to the existing data for the same azimuthal angle $\chi$. The expense in calculation time and implementation effort needs to be traded off carefully against the chance to reduce the error in $S$ from $\frac{\sigma_{S}}{S}=0.3$ to an unknown value. A more sophisticated fitting bears the risk of local minima in the fitting procedure and would result in a non-deterministic approach.

Multiple streaks If two or more streaks are present, the Fourier transform has still significant contributions in the even modes. The anisotropy parameter is therefore still high in this case. As only one angle is determinded for the strongest peak in the azimuthal intensity distribution, the smaller peaks are not accounted for. In order to cover this case, it should be possible to rerun the algorithm on a reduced dataset, which is limited to the angles that were not within a certain distance around a detected peak. As only a small percentage of the diffraction pattern carries such a signal, the missing side peaks orientation is tolerable.

Calculation time The current implementation in matlab uses two for-loops for the coordinate transform. Matlab operations are often quicker, if these loops are rewritten to matrix operations. This is generally possible, and should be tested if fast scanning SAXS experiments require a faster live imaging in the future. A similar thing applies for the fast Fourier transform algorithm, which is used to determine the full Fourier transform. A matrix operation can be formulated for the first six even Fourier modes and the zeroth, so that unnecessary operations are avoided.

\subsection{Other Definitions of Order Parameters}

Another example for an azimuthal anisotropy parameter is the approach that was proposed by Bunk et al. [81]: The group determined the azimuthal intensity distribution without reweighting along $q_{r}$ and fitted a function $f_{b}(\chi)=A+B * \sin (2 \cdot \chi+C)$ to the distribution. The ratio $S_{\text {sine }}:=\frac{B}{A}$ between amplitude $B$ and vertical offset $A$ of the sine serves as anisotropy parameter there. This fit resembles the Streak Finder case for $N_{S F}=1$. Fig. 4.7 a) compares the two approaches, the azimuthal intensity distributions and resulting fits are shown for one dataset. The approach of Bunk et al. gives comparable results for some regions (apparently for horizontally oriented diffraction streaks) of the test data, but differs for other regions (containing vertical ones). Fig. 4.7 b) shows diffraction 
patterns with high anisotropy, it illustrates that the Streak Finder is a better choice for these data obtained from $D$. discoideum. To dicuss the advantage of the Streak Finder algorithm, the inverse Fourier transform of the Fourier modes considered for the Streak Finder and for the fit of Bunk et al. is plotted in Fig. 4.7, along with the (weighted) azimuthal intensity distribution for both approaches. The absolute of this Fourier filtered signal of the Streak Finder is much closer to the data than the sine. A sine-shaped signal, that actually matches the sine function of this fit yields the same anisotropy parameter $S_{\text {sine }}$ as a much sharper peak. Data with sharp peaks should thus be characterized by the Streak Finder $S_{N_{S F}}$.

An anisotropy determination presented by Weinhausen [11] binarizes the diffraction data by thresholding, i. e., all pixels with bigger values than the chosen threshold are 1, else 0 . In addition, a circular region of the central stop is filled with ones. The resulting silhouette is fitted with an ellipse. The circular centre is important to ensure, that scattering-free far-field distributions remain anisotropic. The eccentricity serves as an anisotropy parameter. The major difference to the Streak Finder approach is the thresholding, which is not well applicable to the data presented in this work, as the overal intensity varies between empty sample regions, cell body, and cell perimeter. An advantage of the Streak Finder is that an artificial isotropic filling the centre is not needed, which can influence the fitted ellipse in case of anisotropic signal present. In addition, the calculated Fourier transform allows to adapt the parameter definition to the shape of $I_{r e l}\left(n_{\chi}\right)$ and relative intensity variations are taken into account, where the strongly nonlinear binarization reduces the intensity to a parameter dependent contour. 


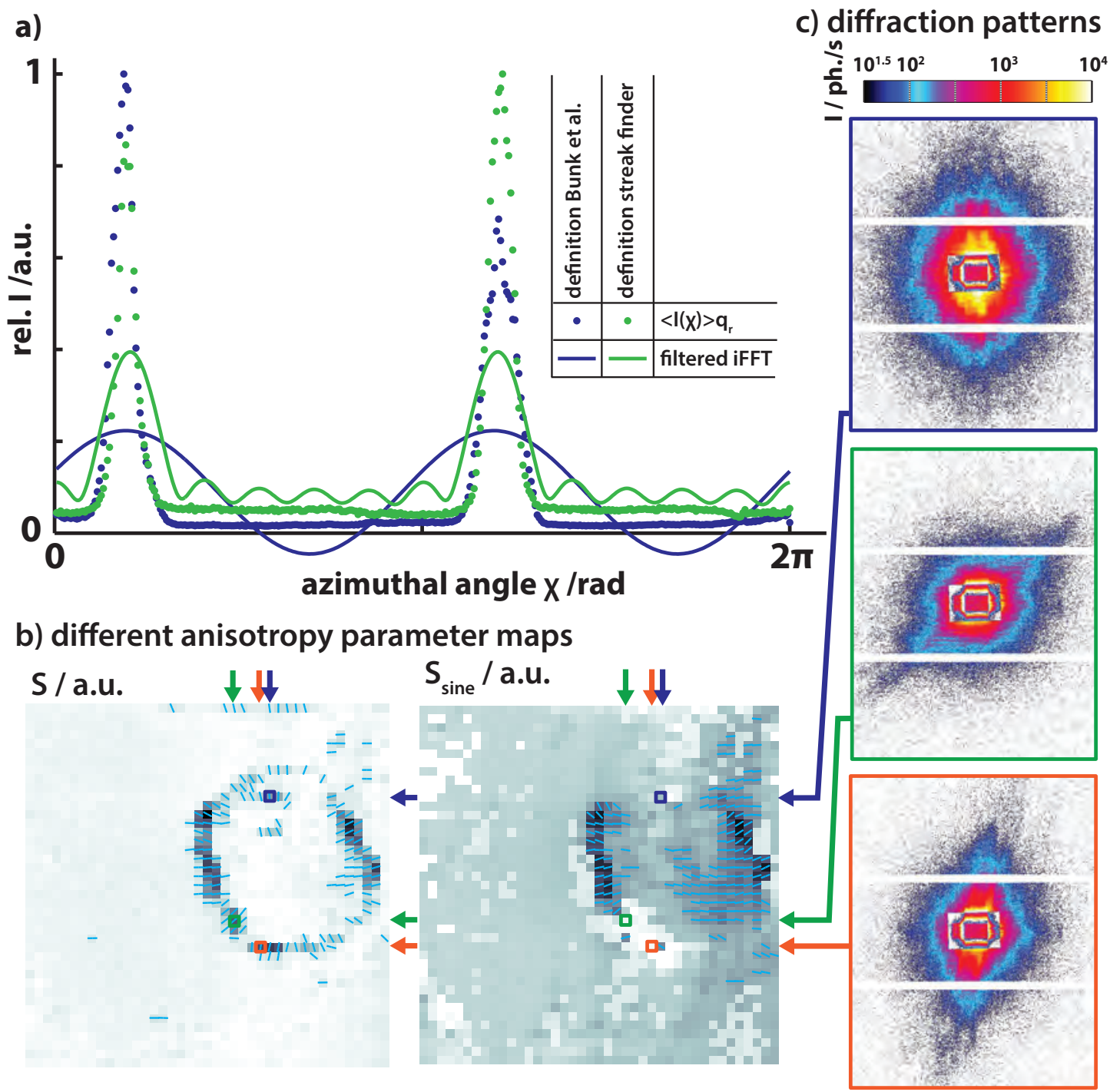

Figure 4.7.: Comparison of anisotropy parameter definitions. a) presents results from the data reduction schemes and fits as presented by Bunk et al. [81] and by us [16]. b) shows anisotropy maps from a $D$. discoideum dataset (frozen-hydrated sample, scanning SAXS at GINIX with $I_{0}=10^{11} \mathrm{ph} . / \mathrm{s}$ in a focus of $326 \mathrm{~nm}(\mathrm{~h}) \times 392 \mathrm{~nm}(\mathrm{v})$, and a dwell time of $0.1 \mathrm{~s}$ for each exposure) dataset from the definitions. Three pixels are outlined, where the two methods lead to different results. c) the corresponding far-field diffraction patterns clearly indicate that considerable anisotropy is present in these datapoints, that is not well-represented in the $S_{\text {sine }}$ map. 


\section{Results}

\subsection{Reciprocal Space}

This section (5.1) is an excerpt from the publication $[16]^{1}$.

Next, we address the analysis of the diffraction patterns in reciprocal space. We distinguish between the azimuthally averaged signal $I_{\text {cell }}\left(q_{r}\right)$ as a function of momentum transfer

$$
q_{r}=\sqrt{q_{y}^{2}+q_{z}^{2}}
$$

where $q_{y}$ and $q_{z}$ denote the horizontal and vertical components of the momentum transfer in Cartesian coordinates. These coordinates are used to describe diffraction signals summed over all pixels in the cell. The two-dimensional pattern $I\left(q_{r}, \chi_{s}\right)$, used for single diffraction pattern, can show anisotropic features reflecting the local orientation in the sample (compare the data shown in Fig. 5.1 and Fig. 5.2, respectively). To represent the signal averaged over the ROI and the azimuthal angle $\chi_{s}$ (Fig. 5.2 (a)), we use masking schemes to regroup and sum diffraction patterns, e.g., to generate representative average background and average (regional) cell signals, similar to Weinhausen et al. [82].

Averaged $I_{\text {cell }}\left(q_{r}\right)$ from entire cells The diffraction patterns of a given scan are first grouped into those obtained from pixels inside [a cell] and those from pixels outside. This information is readily available from a darkfield map (Fig. 5.1 (a)), where the outline of the cell is marked by a line, based on the high cellular SAXS intensity. The diffraction patterns from within and outside the cell are then averaged separately, as illustrated in Fig. 5.1 (b), showing (left) the cellular signal and (right) the background with residual scattering due to chamber, windows, air scattering, amorphous ice, or water. The average is over all data points in the cell regardless of the local anisotropy observed at the periphery of the cell. In the background signal, a residual scattering contribution from KB tails is discerned to the right of the centre, indicating some imperfection in the upstream cleaning optics (apertures). Finally, an azimuthal average over the angle

$1 \quad$ M. Priebe, M. Bernhardt, C. Blum, M. Tarantola, E. Bodenschatz, and T. Salditt: Scanning X-Ray Nanodiffraction on Dictyostelium discoideum. Biophysical Journal, 107(11):2662-2673, 2014. 
$\chi_{s}$ results in a radial intensity decay $I\left(q_{r}\right)$ for background and cell, respectively (Fig. 5.1 (c)). Subtraction of the background curve yields the background corrected averaged scattering signal from the cell $I_{\text {cell }}\left(q_{r}\right)$. Two conclusions can be drawn immediately:

1. The SAXS signal of the cells is substantially higher than the background, i. e., it can be recorded for up to three orders of mangnitude in intensity, reaching the background level at $q_{r} \simeq 0.5 \mathrm{~nm}^{-1}$ which corresponds to a resolution of $\Delta_{R S} \simeq$ $6.3 \mathrm{~nm}$. This resolution in reciprocal space must be distinguished from the real space resolution $\Delta_{S T X M}$ of the scanning transmission x-ray microscopy (STXM) maps given by the beam size or step size.

2. Compared to conventional SAXS from macroscopic suspensions or solutions, the background subtraction can be performed without any free parameters. Whereas variations such as between a capillary with sample and without sample, or one with self-absorption in the sample, in most cases require scaling of signal to noise, the nano-SAXS curves are in perfect registry, reflecting the uniform and identical background on these length scales.

The averaged cellular scattering curves $I_{\text {cell }}\left(q_{r}\right)$ do not show any peaks or modulations. Instead, they show a monotonic decay, which is well described by a power-law function, resulting in a characteristic exponent that can be extracted from a least-square fit. The corresponding graphs can be regarded as a spectral power density of the cell, measured here in the native state without staining or slicing. This power-law decay is in agreement with previous nano-SAXS studies on bacteria [80] and on eukaryotic cells $[10,82]$. Note that in Weinhausen et al. [82], where the signal was mainly attributed to overexpressed intermediate fillaments, two power-law regimes where observed; however, here, $D$. discoideum seems to be well described by a single exponent. Many cells are analysed and the corresponding exponents will be compared further below for the different preparation states (see Fig. 5.5 (c), later).

Streak profiles: In several pixels of the cell, in particular at the cellular boundary, highly anisotropic diffraction patterns have been observed[, in cells of all preparation types]. These anisotropic patterns typically show a single streak extending over the entire detector (Fig. 5.2 (a)). The streak width corresponds to the width of the central beam. This pattern indicates a fibre- or rodlike scattering object, with the fibre axis perpendicular to the streak.

We proceeded by extracting the fibre orientation and performing an empirical analysis of the intensity decay $I\left(q_{r}\right)$, to provide a basis for future analysis that would include 
model fitting, once additional reference data become available. To this end, we first define the ROI around the streak, assessing the orientation angle $\chi_{s}$ of the streak, a lower $q_{\min }$ and upper $q_{\max }$ bound for $q_{r}$, and a width $w$ in the direction perpendicular to the streak, and plotted it as a function of $q_{r}$ (black curve in Fig. 5.2 (b)). The dead regions of the detector (between the three modules) cause missing values in the streak profiles. The position of the gaps varies according to the orientation $\chi_{s}$ of the streak (Fig. 5.2 (c)). Background signal is determined from averaging the remaining diffraction patterns along the azimuthal angle $\chi_{s}$ (red curve in Fig. 5.2 (b)). Fig. 5.2 (c) shows typical profiles after background subtraction, each from different locations within the same cell, as recorded during the same raster scan. The modulations in the streak profiles indicate positional order in the oriented (fibre) structures. Note the diversity of modulations, which could be caused either by local variation in the structure or by rotations of the same structure around the fibre axis. The signal of a single fibre is probably to weak to be observed, but a fibre bundle of actin may possibly explain the observed data.

As a first attempt to parameterize and model this fibre diffraction pattern, one can first proceed as in standard conventional SAXS. In the coordinate system $X, Y, Z$ attached to the fibre bundle with fibre axis $Z$, and reciprocal space coordinates $Q_{Z}$ and $Q_{\|}:=\left(Q_{X}^{2}+Q_{Y}^{2}\right)^{0.5}$, the fibre symmetry (i. e., azimuthal isotropy of the single filament) results in the usual factorization into a single filament form factor $F$. It has a structure factor $S F\left(\mathbf{Q}_{\|}, \mathbf{Q}_{Z}\right)$, which reflects the azimuthal plane positions $\mathbf{R}_{\mathbf{j}}$ of all $1 \leq j \leq N$ filaments in the bundle

$$
I(\mathbf{Q}):=\left|F\left(Q_{z}, Q_{\|}\right) S F\left(Q_{X}, Q_{Y}\right)\right|^{2}=\left|F\left(Q_{z}, Q_{\|}\right) \sum_{j}^{N} \exp \left(\imath \mathbf{R}_{\mathbf{j}} \cdot \mathbf{Q}_{\|}\right)\right|^{2} .
$$

For matter of simplicity, the actin filaments can be modeled as Gaussian cylinders,

$$
F\left(Q_{z}, Q_{\|}\right)=\frac{1}{\sqrt{2 \pi} L_{P}} \exp \left(-0.5 Q_{Z}^{2} L_{P}^{2}\right) 2 J_{1}\left(R Q_{\|}\right) /\left(R Q_{\|}\right)
$$

with $L_{P}$ a parameter associated with the persistence length, i. e., characterizing the effective length of the filament, and $R$ associated with the radius ${ }^{2}$. The model could be extended to more sophisticated models, taking into account the structure of actin including its helicity, [either based on coarse grained representations of electron density [83] or even a fully atomistic model.] As detailed in [B], the intensity distribution in the reciprocal

$\overline{2}$ In our publication [16] we wrote down the zeroth Bessel function $J_{0}$, where the first Bessel function $J_{1}$ should be used (see, e.g., [?]). The simulations were performed with the correct function $J_{1}$, the typo is corrected here. 
plane probed by the two-dimensional detector $I\left(q_{y}, q_{z}\right)$ (corresponding to an equidistant grid in small angle approximation) can then be calculated based on a rotational variable transformation involving a rotation angle $\varphi$ around the fibre axis $Z$, a tilt angle $\vartheta$ of the fibre axis with respect to the optical axis $x$, and a rotation corresponding to the streak angle $\chi_{s}$. Hence, the parameters $R, L_{P}, \varphi, \vartheta$, and $\chi$ along with the filament positions $\mathbf{R}_{\mathbf{j}}$ specify the diffraction pattern.

For illustration, typical streak profiles obtained in this simplistic model are shown in Fig. 5.3 , which mainly served to illustrate the diversity of the diffraction streaks obtained and the dependence on the fiber angles. Aside from the fibre orientation angles, information about the structure of these bundles is particularly contained in the modulation of the intensity decay in the direction perpendicular to the fibre axis, i. e., the streak profile $I\left(q_{r}\right)$ on the detector. Finally, in a nano-diffraction experiment, an important further step is necessary. To account for the finite-size illumination and its deviation from a perfect plane wave, the final intensity pattern of a nano-diffraction experiment $I_{n d}$ is only obtained after a convolution with the probing wavefront $P$, i. e., $I_{n d}=|(S F \cdot F) * P|^{2}$. This last step is responsible for the visible replication of relatively square far-field pattern of the KB in the diffraction beam. However, the approach sketched above was still found to be intractable without further reference data of in vitro cross-linked actin fibres, in view of the large configurational space corresponding to the $\mathbf{R}_{\mathbf{j}}$, and compared to the diversity of experimental patterns observed in the cells.

\subsection{Real Space}

This section (5.2) is an excerpt from the publication $[16]^{3}$.

Characterization of cryogenic samples: Three different scans on frozen-hydrated $D$. discoideum cells are shown in Fig. 5.4 using darkfield and DPCy contrast, complemented by the anisotropy parameter $S$. All cells are clearly visible in darkfield and DPCy, as is the cell contour in the Streak Finder maps. The cell body can be recognized from its stronger scattering in the darkfield, and a stronger primary beam deviation in the DPCy contrast. The relative scattering intensity (darkfield) ranges from $I / I_{0}=[2-7] \cdot 10^{-5}$. The DPCy maps color encode the deflection of the primary beam on the detector in the unit detector pixels with typical ranges of $1.5-7$ pixels. Due to the position of the semitransparent beamstop, it is not possible to accurately measure the position of the primary beam (see [Fig. 3.2]). These values therefore have to be interpreted as a relative value rather

$3 \quad$ M. Priebe, M. Bernhardt, C. Blum, M. Tarantola, E. Bodenschatz, and T. Salditt: Scanning X-Ray Nanodiffraction on Dictyostelium discoideum. Biophysical Journal, 107(11):2662-2673, 2014. 


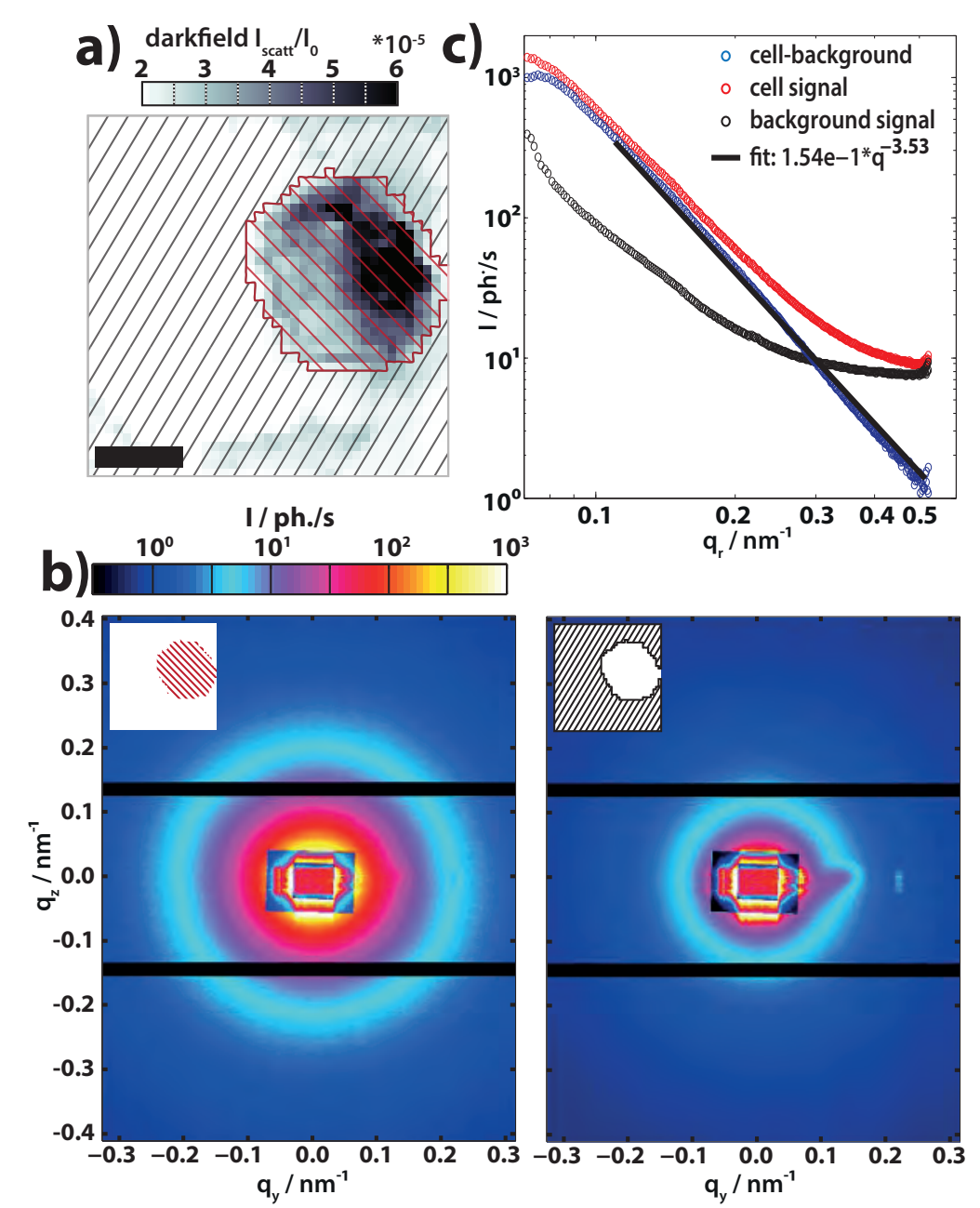

Figure 5.1.: (a) Based on a scans darkfield map, two subsets of pixels are defined, those with scattering from the cell (hatched in red) and the surrounding ones (hatched in black). (b) The diffraction pattern of these subsets are averaged separately for the (left) cell and (right) background region. (c) Azimuthal averaging yields (red and black) two curves $I\left(q_{r}\right)$ for the subsets. The cell signal is corrected for background effects by subtraction of the background signal. The resulting blue curve can be fitted with a power-law function, thus characterising the curve with a single exponent. Material of this figure and caption was also published in [16]. 

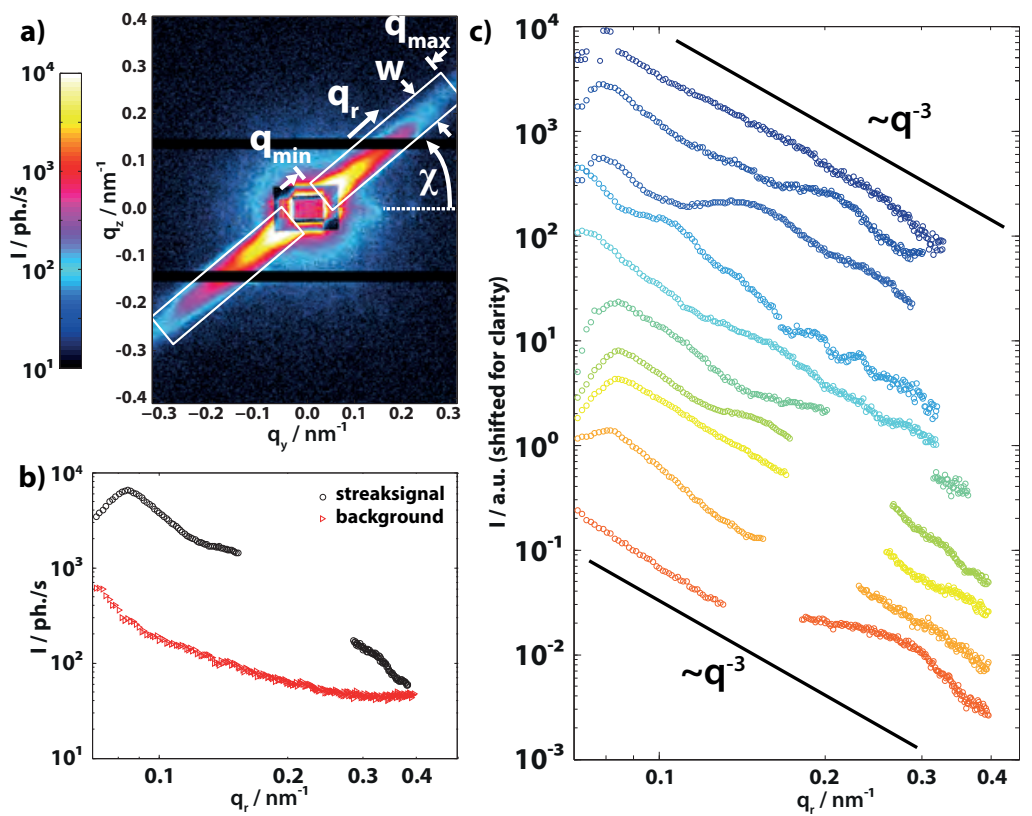

Figure 5.2.: Comparison of the $I\left(q_{r}\right)$ profiles in streaky diffraction patterns. (a) Typical example for anisotropic scattering patterns. The streak intensity profile is read out by averaging the intensity perpendicular to the streak. The readout region is defined by the azimuthal angle $\chi_{s}$, the inner $q_{\min }$ and outer $q_{\max }$ scattering vector $q_{r}$, and the width perpendicular to the streak $w$. The remaining part of the detector image is used for background correction by averaging along constant radii over the azimuthal angle. (b) Resulting streak and background signal from (a). A gap in the data corresponds to the dead regions of the detector. The difference $I_{\text {streak }}\left(q_{r}\right)-I_{\text {background }}\left(q_{r}\right)$ represents the intensity distribution in the streak pattern. (c) Profiles $I\left(q_{r}\right)$ of different streak patterns measured on the same cell. The plots are shifted for clarity and a power-law decay $\propto q^{-3}$ is drawn as a guide to the eye. Material of this figure and caption was also published in [16]. 
a)

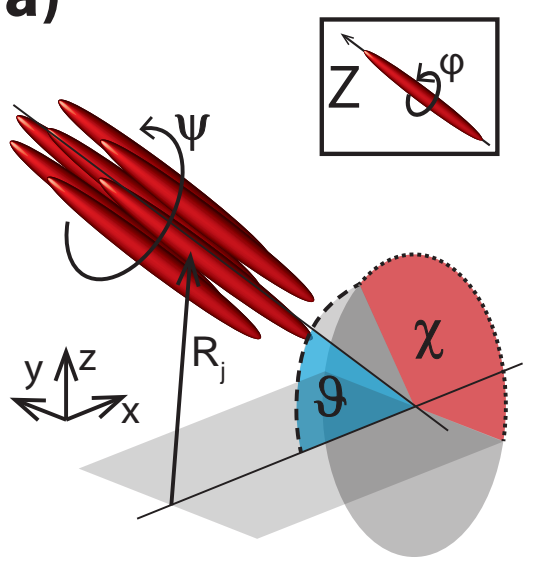

b)

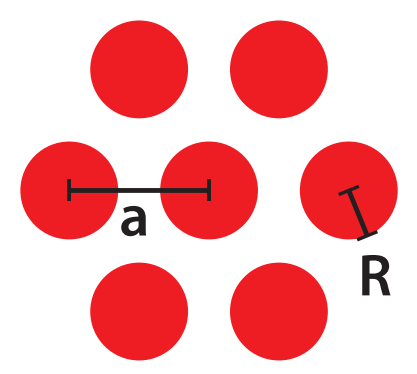

C) $1 /$ a.u. $10^{0} 10^{-1} 10^{-2} 10^{-3}$

$a=33 \mathrm{~nm}, R=7 \mathrm{~nm}, L_{\mathrm{p}}=100 \mathrm{~nm}$

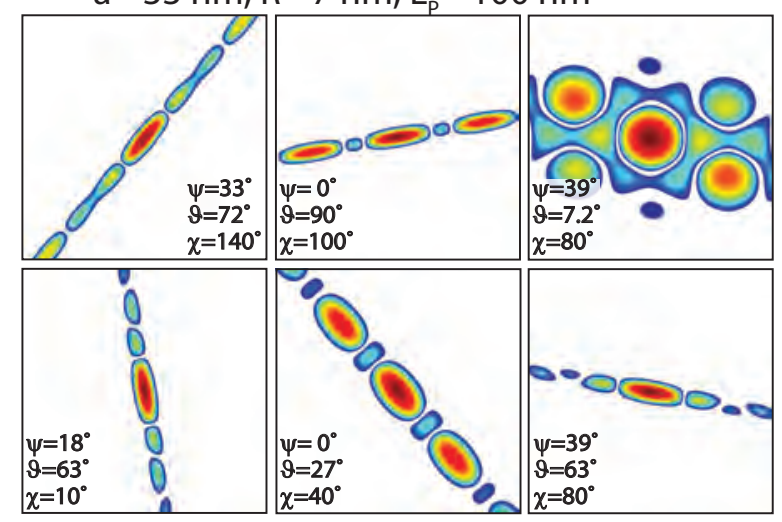

d) I/ a.u. $10^{0} 10^{-1} 10^{-2} 10^{-3}$

$a=12.4 \mathrm{~nm}, R=3.5 \mathrm{~nm}, L_{p}=100 \mathrm{~nm}$

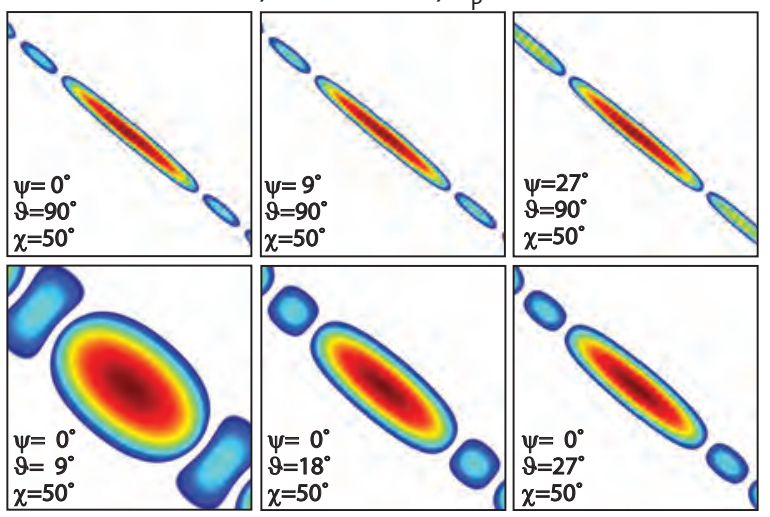

Figure 5.3.: (a) Sketch of an arbitrarily defined fibre bundle consisting of seven filaments arranged in a hexagonal lattice. The angle $\vartheta$ and the azimuthal angle $\chi$ describe the orientation of the fibre bundle. Together with the bundle rotation angle $\psi$, which is defined in the bundles coordinate system, they determine the far-field diffraction pattern of a given set of filaments. The filaments are defined by their position $R_{j}$ and have an individual angle $\varphi$, defined in the filaments coordinate system. (b) Variables characterising the hexagonal lattice in the plane perpendicular to the bundle main axis. (c) Simulated diffraction patterns calculated from seven rods. The rods are aligned in a hexagonal lattice. We show six different configurations of the angles $\psi, \vartheta$, and $\chi$, the angle $\varphi$ does not influence the result due to the rotational symmetry of the single rods. For small $\vartheta$, here $\vartheta=7.2^{\circ}$, the hexagonal rod lattice leads to side maxima while the far-field intensity distribution appears streak like for all others $\vartheta \in\left[27^{\circ}-90^{\circ}\right]$. It is easy to see, that the orientation of the far-field intensity distributions can be interpreted directly with regards of the fibre orientation $\chi$. Material of this figure and caption was also published in [16]. (d) Simulated diffraction patterns as in (c), but showing systematic variations of (upper panels) the bundle rotation angle and (lower panels) the angle of the bundle against the optical axis. The q-range of the far-field patterns measures approximately $q_{y, z} \in[-0.4 \mathrm{~nm}, 0.4 \mathrm{~nm}]$. 
than an absolute measurement. The color range of $S$ varies for the measurements shown and lies between $S=[0.04-0.4]$ for Fig. 5.4 (f) and $S=[0.04-2.0]$ for the dataset shown in Fig. 5.4 (i), in which the anisotropic scattering patterns even dominate the corresponding darkfield map Fig. 5.4 (g). Despite the variation of $S$ between different datasets, anisotropic scattering occurs predominantly on the cell perimeter with the orientation perpendicular to it.

Experiments on initially living cells: Scattering experiments were performed on living cells of $D$. discoideum using the x-ray-compatible microfluidic chambers described in Sec. 3. Fig. 5.5 (a) shows the darkfield maps for three datasets scanned subsequently on the same cell to address the interaction of the beam with the sample due to beam damage. The first two raster scans were performed with the shortest possible dwell time of $0.05 \mathrm{~s}$ and the attenuated beam $I_{a t t}=0.23 \cdot I_{0}=2.5 \cdot 10^{10} \mathrm{ph} . / \mathrm{s}$. The third scan was performed with the full primary intensity $I_{0}=1.1 \cdot 10^{11} \mathrm{ph} . / \mathrm{s}$ and long dwell times of $0.5 \mathrm{~s}$. Slight changes are already visible between the first two scans, inasmuch as the scattering intensity increases a little. These are characterized via the background corrected averaged scattering signal from the cell $I_{\text {cell }}\left(q_{r}\right)$, as explained before in Fig. 5.1. The first two datasets coincide except for the region of small $q_{r}$ (see Fig. 5.5 (b)) whereas the last one drops significantly in intensity. Further, the exponent $\gamma$ of a power-law decay $F(\gamma) \propto q_{r}^{\gamma}$ fitted to an inset $0.11 \mathrm{~nm}^{-1} \leq q_{r} \leq 0.42 \mathrm{~nm}^{-1}$ decreases, here from $\gamma=-2.66$ via -2.83 to -3.49 , which we find to be characteristic for beam damage during repeated exposures.

Comparison of preparation protocols and beam damage: Many comparable datasets have thus been analysed and classified by their power-law exponent. All datasets with reasonable signal/noise are represented by their power-law exponent in Fig. 5.5 (c), where they are sorted by their preparation method and dose history. Solid symbols represent scans that were not exposed to more than a search scan (as can be seen in Fig. 3.1 (e), right image). Successive detailed scans are represented by open symbols. Small horizontal lines show the mean value for each subset.

Datasets from initially living cells that were scanned for the first time have not endured any search scans (inasmuch as the cells migrate too quickly for this method), and can act as a reference for all preparations. The power-law exponent is largest in these scans, although the datasets show a wide spreading. The datasets on initially living and frozen-hydrated samples show a strong tendency to a steeper power-law decay from the first to subsequent scans, indicating beam damage. The cryopreparation also shows a shift in the exponent as compared to the initially living cells, an indication that cell 

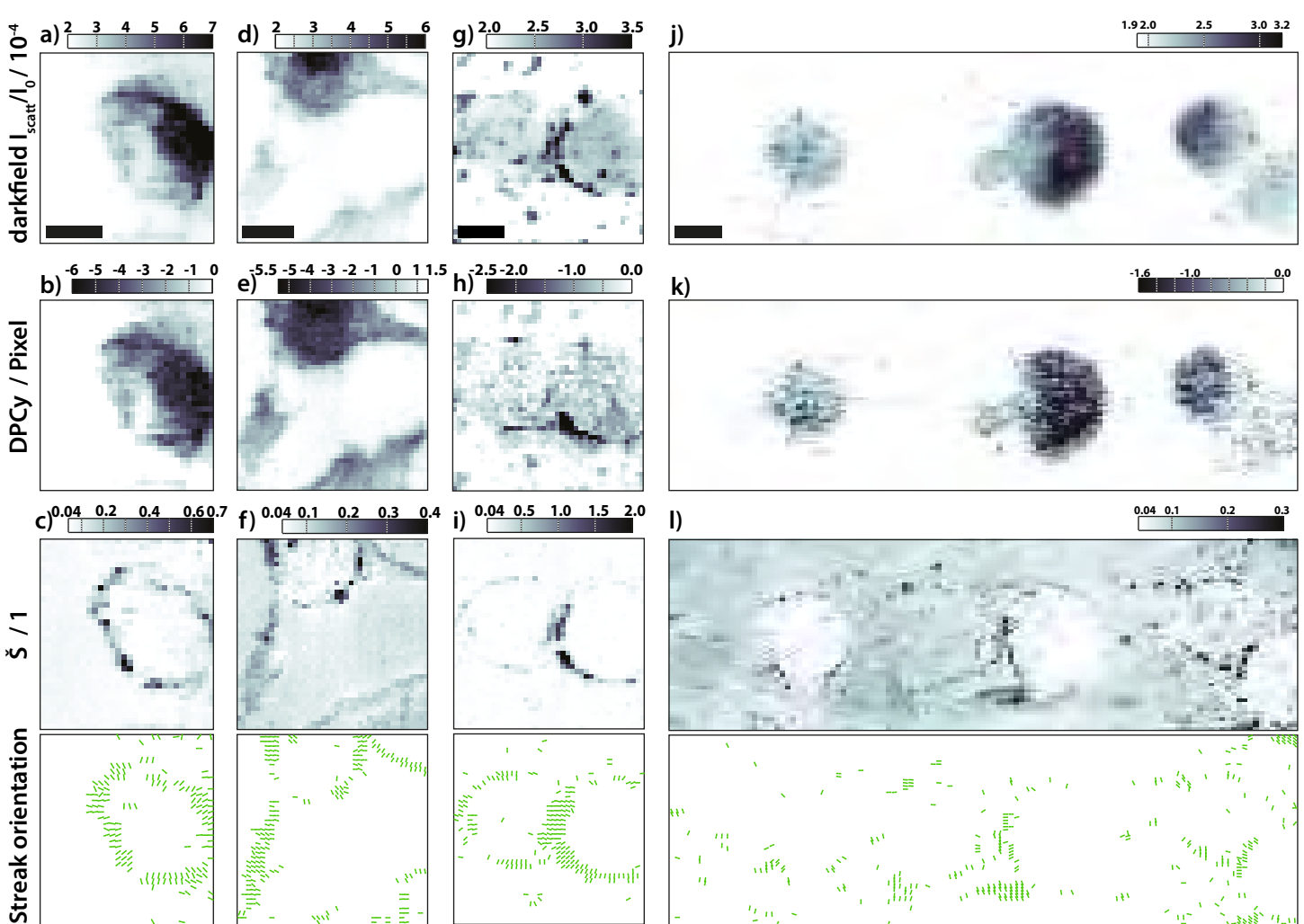

Figure 5.4.: Comparison of four different scans (columns) on cryogenic hydrated cells in three different contrast modi (rows). All measurements were performed at GINIX with $I_{0}=1.1 \cdot 10^{11} \mathrm{ph} . / \mathrm{s}$ and $0.1 \mathrm{~s}$ exposure per data point. The scale bars denote $5 \mu \mathrm{m}$. (a-c) show a typical case often found for single cells where the cell body shows up in darkfield and differential phase contrast (y component: DPCy). Anisotropic diffraction patterns are occuring close to the cell cortex and are standing perpendicular to it. (d-f) Cell with additional elongated structures, that show streaks perpendicular to its elongation direction. (g-i) Two adjacent cells show a comparable behaviour to the single cells in (a-f) but with particularly pronounced streaks occurring at the cell-cell contact region. (j-l) A wider scanning range including three cells that show a relatively low anisotropy parameter. Here, the central cell is of special interest, showing only weak scattering and weak anisotropy, which is still pronounced around the protrusion at the lower left side of the cell. Material of this figure and caption was also published in [16]. 


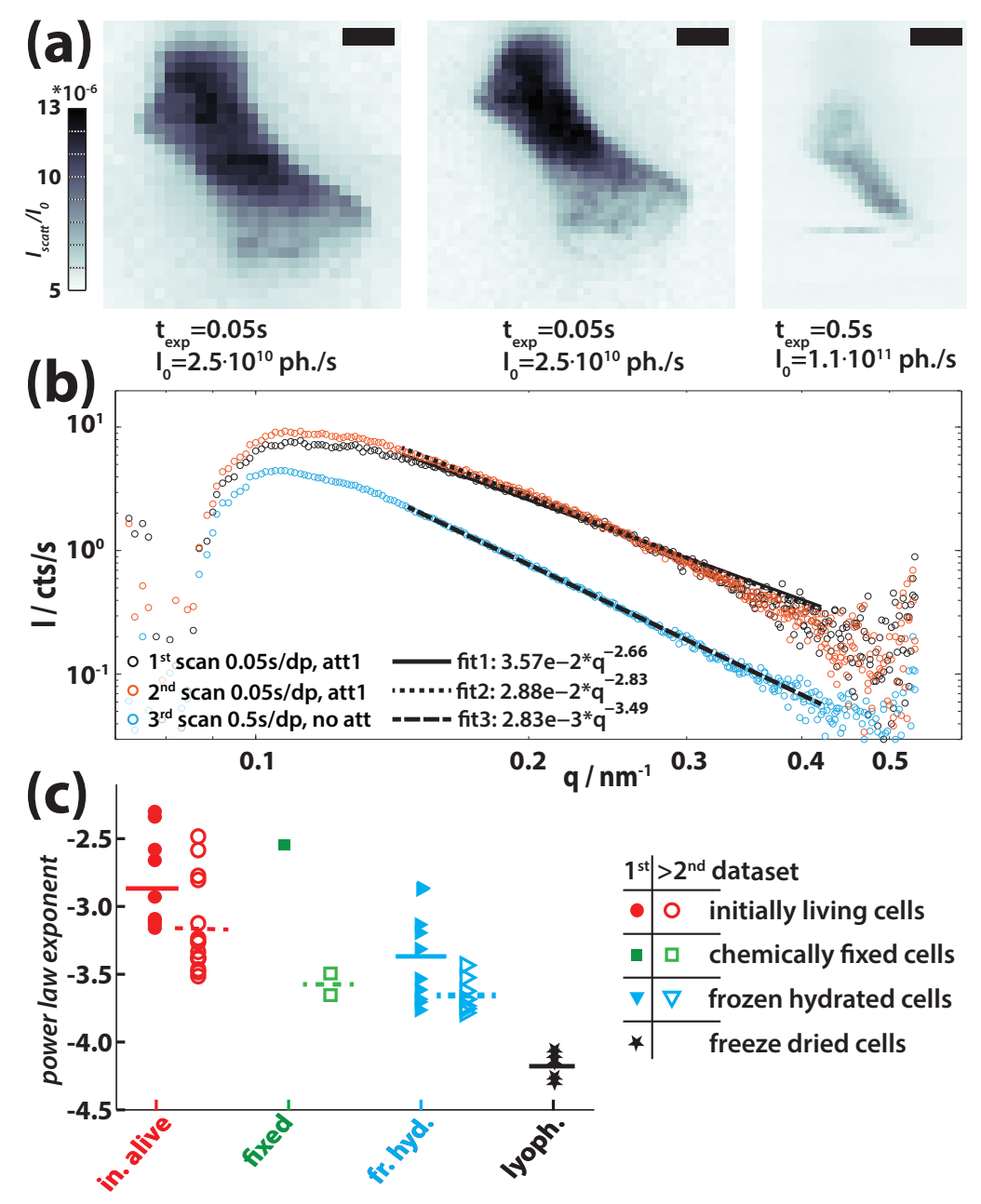

Figure 5.5.: Experiments on initially living cells and comparison of preparation protocols. (a) Darkfield maps of three consecutive scans on an initially living $D$. discoideum cell in an microfluidic chamber. The scale bars denote $5 \mu \mathrm{m}$ and the color axis is adjusted equally. The first and second scan are done with short exposure times and a reduced primary intensity of $I_{0}=0.23 \cdot I_{0, \max }$. All three darkfields show slightly different intensity values, which becomes clearer in (b), background-corrected scattering signal $I_{\text {cell }}\left(q_{r}\right)$ from the cell in panel (a). The second measurement (orange curve) mainly coincides with the first scan's data (in black), but intensity and the fitted exponent decrease significantly towards the long third scan (blue). (c) To compare the influence of preparation methods and measurements on the results, this scheme was applied to many measured cells, namely samples with initially alive cells, with formaldehyde-fixed cells, with cells that were frozen-hydrated (plunge-frozen), and with cells that were plunge-frozen and additionally freeze-dried. (Solid symbols) Scans on cells that have not encountered the dose of a fine scan (like shown in panel (a), unless open symbols are used). (Small lines) Average value for one set of points, solid lines for solid symbols; and dashed lines open symbols. Material of this figure and caption was also published in [16]. 


\section{$\begin{array}{lll}\text { a) } \mathrm{I}_{\text {scatt }} / \mathrm{I}_{0} / 10^{-4} & \text { b) } \mathrm{S} / 1 & \text { c) fluor. mic. }\end{array}$

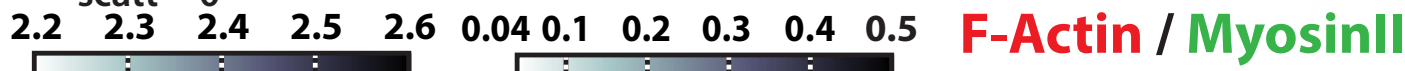
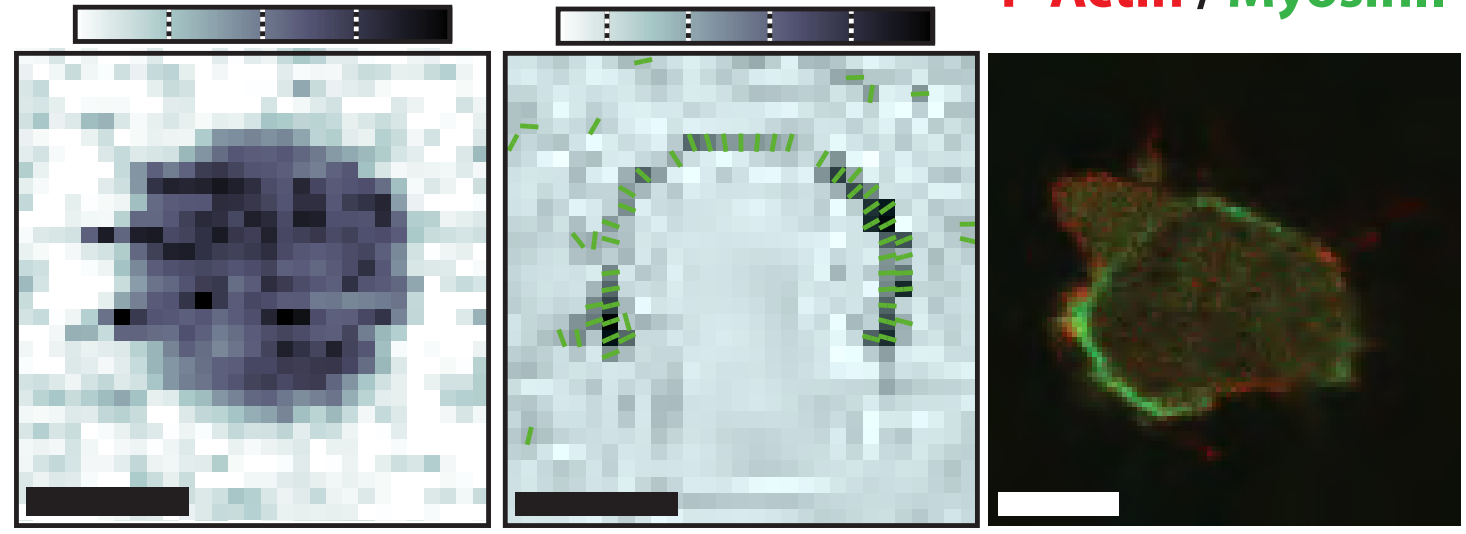

Figure 5.6.: Maps of the (a) darkfield and (b) anisotropy parameter of a frozen-hydrated $D$. discoideum measured at the GINIX with a dwell time of $0.1 \mathrm{~s}$ at $I_{0}=1.1 \cdot 10^{11}$. The scale bars equate $5 \mu \mathrm{m}$. (c) Fluorescence micrograph of a $D$. discoideum (AX2 wild type with LimE-mRFP and myosin-II-GFP label scanned sequentially line by line) imaged with an Olympus IX81 Confocal Laser Scanning Microscope. The cell is moving towards the top right of the image. On the top left, a pseudopodium is identified by the high actin concentration. The rear end, facing the lower left corner, shows a high concentration of myosinII. This is typical for the contractile actomyosin arc at the rear end of cells during the attachment-retraction cycle. We often find that the oriented signal of this network shows one or more weaks spots resulting in a $\mathrm{C}$-shape, as can be seen in (b). Material of this figure and caption was also published in [16].

microstructure has actually been altered (opposite to what is commonly believed $[67,68]$ ) throughout the preparation process. As expected, the intensively processed lyophilized samples show the smallest exponent and vary significantly from all other prepration techniques. Chemically fixed cells have been shown recently to differ from their native state [82], but more statistics is needed here to comment on it.

\subsection{Results from ID13}

Fig. 5.7 shows characteristic datasets from STXM scans on a freeze-dried sample of $D$. discoideum $^{4}$. A dataset from a scan covering the whole cell is shown in (a) and a more detailed scan was performed on the lower left region of the cell, shown in (b). The latter has a mesh size of $100 \mathrm{~nm}$ between adjacent diffraction pattern recordings which is the

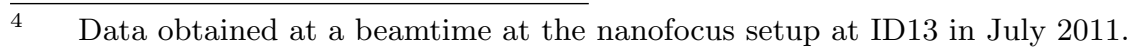


highest real space resolution shown in this work and also the smallest possible, given the beam dimensions of $(h \times v): 140 \mathrm{~nm} \times 110 \mathrm{~nm}(\mathrm{FWHM})$. The diffraction patterns on the cell perimeter show strong anisotropy, with the main orientation perpendicular to the cell wall. This is in accordance with the results obtained from frozen-hydrated samples, presented earlier in this work. The ROI in (b) contains a circular feature, a region of $0.5 \mu \mathrm{m}$ in diameter, where the scattering is less intense as compared to the surrounding far-field patterns. Also, several of the diffraction patterns around this feature show anisotropic intensity distributions that are perpendicular to the boundary. Whether this substructure has natural origin (e.g., a vacuole) or is a drying artefact cannot be inferred from the data, but small circular structures have been observed in holographic imaging of freeze-dried D. discoideum cells [12].

An typical diffraction pattern from (b) is shown in (c). Above and below as well as on the left and right side of the beam centre region, artefacts from the primary beam are visible (in dark red). The two symmetric spots (1 and 7 o'clock position) indicate elongated and strongly orientated structures in the sample.

Results obtained on a later beamtime at the same setup are shown in Fig. 5.8. The beam size was smaller in this second experiment, so that the scattering volumes do not overlap in scans with $100 \mathrm{~nm}$ step size. The results are consistent with the previously discussed ones in terms of anisotropic scattering signals on the cell perimeter as well as local variations within the cell. Anisotropic scattering is even found inside the cell in Fig. 5.8 (d). A second cell shown in Fig. 5.8 (b,e,g) has pronounced elongated features outside the central cell. The origin of these structures is not entirely clear. The position adjacent to the cell wall could point to residual cellular material from either a neighbouring cell that detached during freeze-drying or cellular extensions. The signal is interesting as an example of multiple streaks which have next to no background signal. However, further analysis is required to comment on the origin of this signal.

Similar substructures as in figures 5.7 and 5.8 could not be observed in frozen-hydrated samples, as, first of all, the contrast in the electron density (cellular components embedded in water as compared to cellular components embedded in air) is weaker for frozenhydrated samples, an in addition the beam at GINIX is $2 \times 2$ as large as the beam at the nanofocus setup at ID13. Some features would thus be averaged out in the larger beam. Some of the neighbouring far-field patterns show strong variations but others appear similar in anisotropy and orientation over $\approx 0.5 \mu \mathrm{m}$, which indicates that structural changes appear on scales of $100 \mathrm{~nm}$ or smaller but also far-ranging correlations are found. A smaller focal size could thus be promissing to reveal local changes in frozen-hydrated samples. 
a)

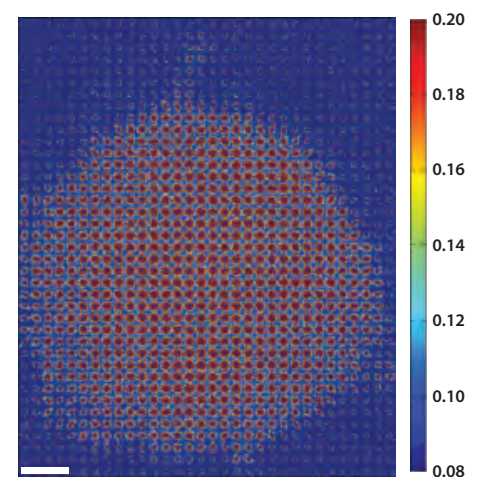

b)

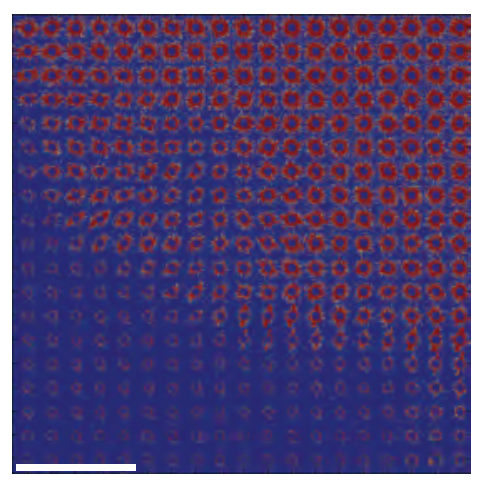

c)

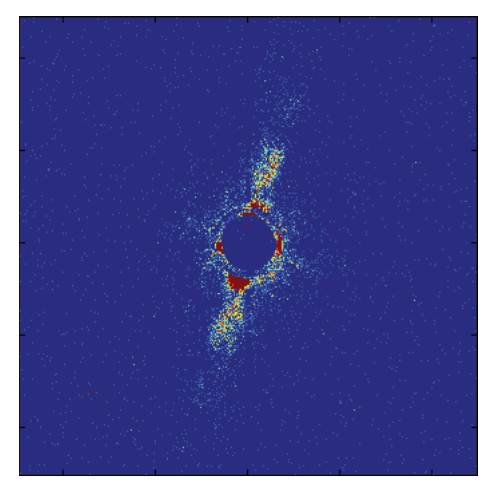

Figure 5.7.: Diffraction dataset from a freezed dried D. discoideum wild-type cell obtained at the nanofocus endstation at ID13, ESRF. (a) Shows the full dataset in a composite view, an inset in the region of interest on the lower left can be found in (b). Finally, distinct anisotropic scattering is visible in many of the (c) single diffraction pattern aquired on the cell perimeter. The scale bars denote (a) $1 \mu \mathrm{m}$ and (b) $0.5 \mu \mathrm{m}$. Dwell time was $1 \mathrm{~s}$ per exposure, the primary beam intensity was $I_{0}=3 \cdot 10^{9} \frac{\mathrm{ph}}{\mathrm{s}}$.

\subsection{Ptychographic Phase Imaging}

Ptychography is an x-ray imaging technique that allows the reconstruction of phase shift and absorption of the imaged object as well as the full phase and intensity map of the probing wave function. Phase information is generally not accessible due to the fact that photon detectors measure the absolute squared of the electrical field $I=|E(x, t)|^{2}$. Phase information is not captured, this fact is known as the phase problem. A measurement suitable for ptychographic reconstruction is done in the same way as a STXM measurement, by raster-scanning the sample, with the difference that a certain overlap between adjacent iluminated spots on the sample is required. Diffraction patterns obtained at neighbouring positions thus partly share the same information. With this constraint an iterative algorithm is able to restore the missing phase information.

Detailed information can be found in, e.g., [12,84].

During the beamtime at the cSAXS beamline, we managed to perform ptychographic recordings of freeze-dried as well as frozen-hydrated samples. Fig. 5.9 (a-c) show reconstructions from $D$. discoideum cells. The cells shown in $(\mathrm{a}, \mathrm{b})$ are frozen-hydrated. Far-ranging structures are visible on or within the cell, but these do not resemble anything to be expected within $D$. discoideum. These cells probably suffer from preparation artefacts. Problems during cryo-plunging, heating up above the glass transition temperature or (for (a)) an ice crystal from the liquid nitrogen bath lay down on it. (c) shows a freeze-dried specimen of $D$. discoideum. Many more internal structures are visible in 


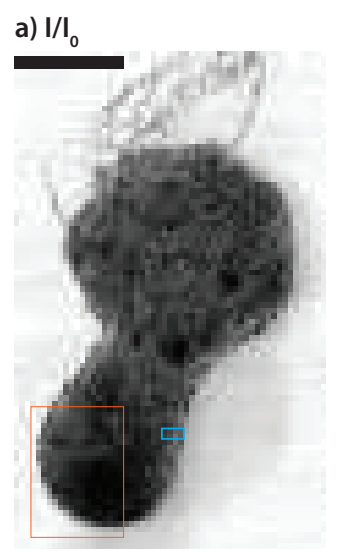

d)

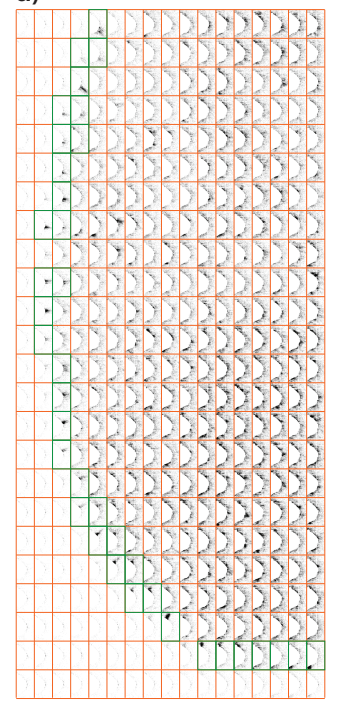

f)

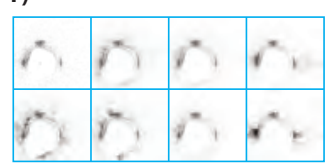

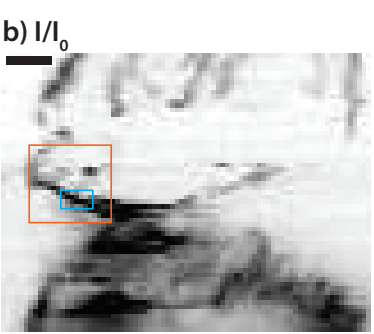

c) legend for d)-e)

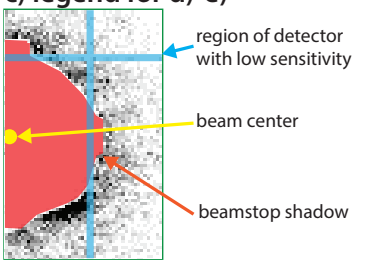

e)

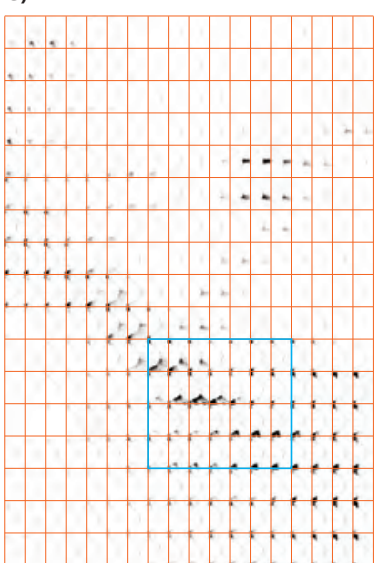

g)

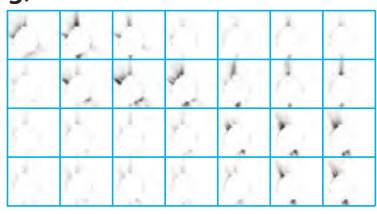

Figure 5.8.: Results from scanning nano-SAXS experiments on freeze-dried D. discoideum cells. a) Darkfield map of whole cell, that shows elongated features on top. Step size was $250 \mathrm{~nm}$ dwell time $0.4 \mathrm{~s}$ per scanpoint, the scale bar equates $5 \mu \mathrm{m}$. b) shows a detailed scan covering the upper tip of the cell in a) and above, using $100 \mathrm{~nm}$ steps between adjacent scan points and a dwell time of $0.4 s$. The scale bar indicates $1 \mu \mathrm{m}$. c) explains the different features found in the composite images d) and e). d) Composite image from orange inset in a) with high anisotropy close to the perimeter of the cell. In particular, many streaks are found that are oriented perpendicular to the cell perimeter (marked in green for clarity). e) same for the inset scan in b), here the anisotropy is even more pronounced, but is not oriented perpendicular to the structures visible in the darkfield b). f) and $\mathbf{g}$ ) show composites of smaller insets indicated in blue in a) (and (b), respectively), here larger cutouts are used to give more detailed impression of the data. Both datasets were obtained with the nanofocus extension of ID13 (ESRF, Grenoble), using a set of nano-CRLs, that focus the incoming beam down to well below $100 \mathrm{~nm}$ in both dimensions, as determined with Ptychography. The intensity of the primary beam was $I_{0}=1.6 \cdot 10^{9} \mathrm{ph} . / \mathrm{s}$. 
this preparation, which has been observed earlier already, e.g., [12]. The samples in (d-f) show groups of the bacterium D. radiodurans, prepared by my colleague Robin N. Wilke. Again, (d,e) are frozen-hydrated and (f) shows the reconstruction from a freeze-dried specimen. In contrast to $D$. discoideum, the bacterial cells do not show pronounced internal structures except from one region of higher phase shift for each cell, that originates from the genome. As procaryotic cells do not have a comparted organisation, this is not surprising. In good agreement with previous observations is the phase shift that ranges $-0.2 \mathrm{rad} . .-0.25 \mathrm{rad}$ for frozen-hydrated samples and is $\approx 0.5 \mathrm{rad}$ for freeze-dried samples. This is again a result from the lower electron density contrast for frozen-hydrated samples.

Now that we successfully reconstructed frozen-hydrated $D$. discoideum cells, we plan to combine this approach with nano-SAXS. As described in our publication [16], the required coherence for Ptychographic recordings can be achieved by closing slits. A ptychographic scan would allow to first measure the electron density of the cell and then raster scan with a less coherent beam to record the full information available from the reciprocal space (with beamstop, if required).

\subsection{Fluorescence Measurements}

An excitation energy of $E_{\lambda} \geq 10 \mathrm{keV}$ is sufficient to excite the K-shell electrons (corresponds to the orbital with $n=1$ for $E_{n, m, l}$ and thus $l=0$ and $m= \pm \frac{1}{2}$ ) of many physiologically relevant elements like $\mathrm{K}, \mathrm{Na}, \mathrm{Ca}, \mathrm{P}, \mathrm{Cl}, \mathrm{S}, \mathrm{Zn}, \mathrm{Fe}$, and $\mathrm{Cu}$ [85], but also heavier elements. This unstable state decays by transition of electrons into lower energetic orbitals and simultaneous emission of a photon. The photon energy corresponds to the energy difference of the orbitals.

Elemental maps are of particular interest, as metal ions are present in one third of all proteins known today, where they act as cofactors [85]. These metals can thus act as natural markers. Giewekemeyer et al. [86] have already performed x-ray fluorescence measurements on $D$. discoideum, they were able to identify lines of phosphor, potassium, calcium, and zinc.

The energy calibration was done with a fitting routine of PyMCA [71] using the peaks of $E\left(A r_{K \alpha}\right)=2.958 \mathrm{keV}, E\left(K_{K \alpha}\right)=3.314 \mathrm{keV}, E\left(C r_{K \alpha}\right)=5.415 \mathrm{keV}, E\left(F e_{K \alpha}\right)=$ $6.404 \mathrm{keV}$, and the elastic peak of the primary beam $E_{\text {elast. }}=7.90 \mathrm{keV}$ (for fluorescence emission energies, see [87]). The fine-structure splitting of the fluorescence lines (due to the electron spin) is not resolved in these experiments. Therefore, more intense energy 
a) frozen hyd. $D$ d., $\quad \$ 12671$

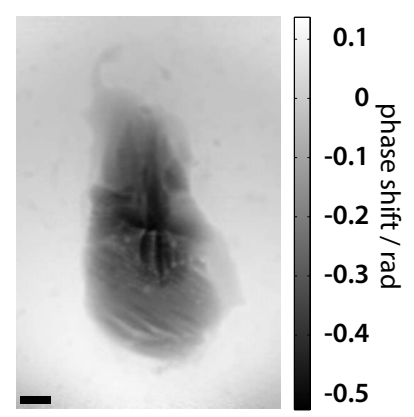

d) frozen hyd. RD.,

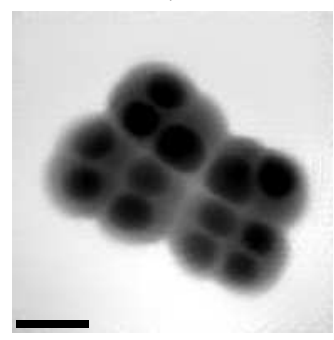

b) frozen hyd. $D d$.

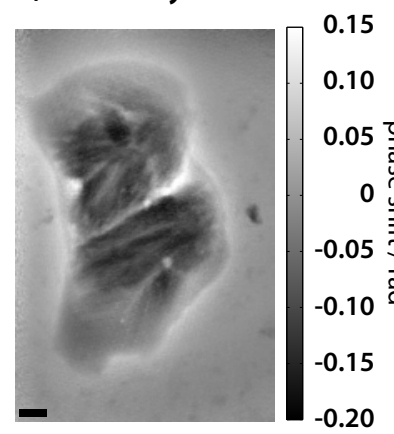

e) frozen hyd. RD,

0.05
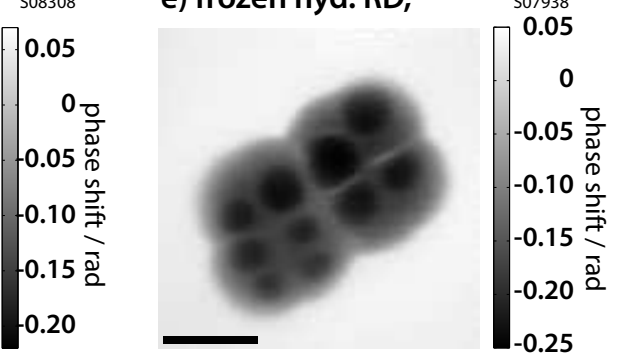

c) freeze dried $D d$,

501219
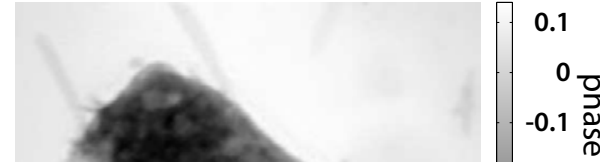

-0.2 告

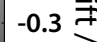

-0.4 ڤั

$-0.5$

$-0.6$

f) freeze dried $R D$., $\quad 506836$

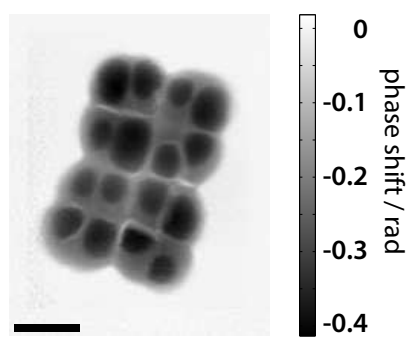

Figure 5.9.: Ptychographic reconstructions of several D. discoideum and D. radiodurans, the contrast of the freeze-dried samples is approximately $\Delta \phi=0 \mathrm{rad} \ldots 0.25 \mathrm{rad}$, while the freeze-dried samples show a higher contrast of $\Delta \phi=0 \mathrm{rad} \ldots 0.6 \mathrm{rad}$. The $D$. radiodurans cells are alike, despite different ranges in the contrast. The $D$. discoideum cells show significantly more structure inside the cells for both, the frozen-hydrated and freeze-dried state.

The ptychographic reconstructions were made with the programs and the support of Robin N. Wilke. All scale bars equate $2 \mu \mathrm{m}$. 
was chosen as a reference value. The fit of the calibration parameters to the conversion in equation 3.1 (repeated below) yielded

$$
\begin{aligned}
& A_{f l}=-4.415063 \times 10^{-4} \mathrm{keV} \\
& B_{f l}=4.8295895 \times 10^{-3} \mathrm{keV} \\
& C_{f l}=1.0289301 \times 10^{-8} \mathrm{keV} \\
& E_{f l}=A_{f l}+B_{f l} N_{\text {channel }}+C_{f l} N_{\text {channel }}^{2}
\end{aligned}
$$

with the unit $\left[E_{f l}\right]=\mathrm{keV}$, as $\left[N_{\text {channel }}\right]=1$.

The peaks found at energies higher than the excitation photon energy $E_{\text {elast }}=7.9 \mathrm{keV}$ are artefacts from the spectrometer (so-called "multi channel analyser"). When two events are not well separated, the spectrometer registers these events as one with twice the energy of the single event, which results in so-called pile-up peaks that are shifted to the double energy of the original peak. These peaks therefore resemble the energy spectrum, but are much lower in intensity.

Spectra of a recording can be regrouped into a ROI and a background region, either based on a STXM-map or an element map from fluorescence data. In Fig. 5.10 an element map of $K_{K \alpha}$ was employed to define (c) ROI and background. The subsets of data are averaged separately and plotted $(\mathrm{d})$. The averaged spectra show increased intensity in the ROI for five peaks. These peaks can be attributed to the characteristic energies of potassium $K_{\alpha}$, potassium $K_{\beta}$, sulfur $K_{\alpha}$, phosphorus $K_{\alpha}$, and aluminium $K_{\alpha}$.

While the first four are easily identified, the aluminium line does not perfectly fit the peak position. Other possible emission lines can be found in the table of x-ray emission lines in the x-ray data booklet [87]. Besides other heavy or physiologically irrelevant elements, bromine emits a $L \alpha$ line just $7 \mathrm{eV}$ from $A l_{K \alpha}$. However, literature research did not reveal any function of bromine in the cell. Also, the origin is unclear as none of the ions is added to the buffers or media. This makes the aluminium line the more likely candidate, considering a secondary effect. Fluorescence lines like the strong $K_{K \alpha}$ emit in $4 \pi$ and can, possibly, excite aluminium that is present in many components of the setup. This is a possible explanation, but needs further investigation.

Despite the rather weak intensity in the spectra, the element lines of $\mathrm{K}, \mathrm{S}$, and $\mathrm{P}$ are visible. Most interesting is complementary distribution of $\mathrm{K}$ and $\mathrm{S}$ within single cells which both show spots of high concentration, but never coinciding. This information cannot be attributed to certain cell components yet but should be tested on more cells. The quality of the fluorescence signal was compromised by technical problems. With an optimised experimetal setting (namely repair of the fluorescence detector, the detection 
a) Average fluorescence signal - full range

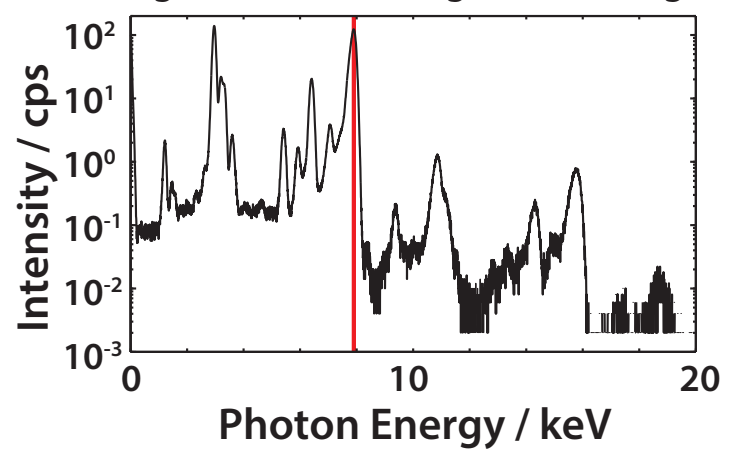

b) $\mathrm{K} K \alpha$ line element map

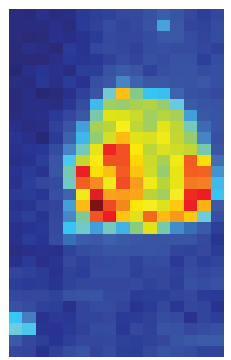

c) masking scheme for d)

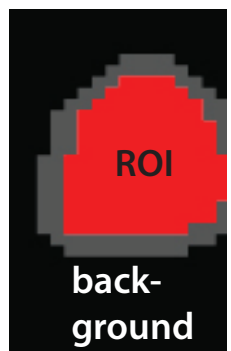

d) Av. fluorescence signal

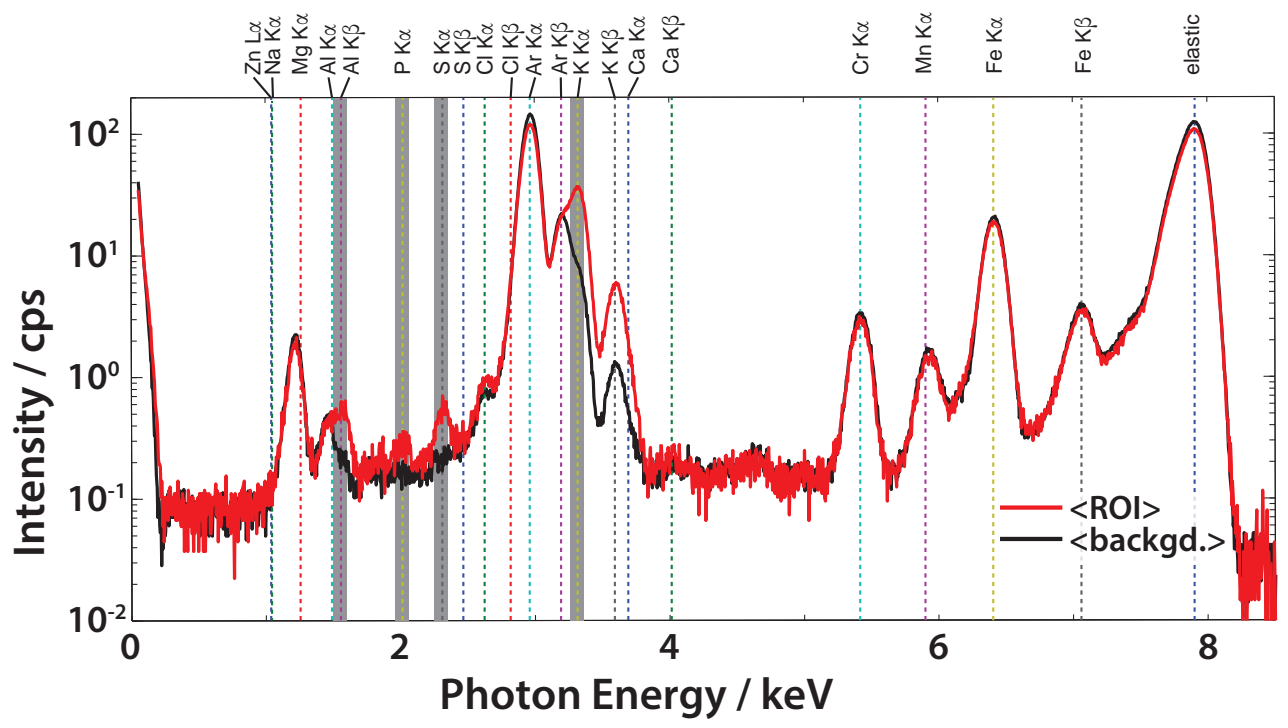

Figure 5.10.: Results from fluorescence acquisition. (a) Typical raw data of fluorescence acquisition with energy scale. The red line marks the energy of the excitation wavelength $E_{\text {elast }}=7.9 \mathrm{keV}$, all peaks at higher energies (right of the red mark) are a result of pile up. (b) Elemental map of the interval $3.26 \mathrm{keV} \ldots 3.36 \mathrm{keV}$ corresponding to the $K_{K \alpha}$ line. A ROI around the cell (c) is definded manually based on this map. (c) Masking scheme applied to dataset spectra obtained from the ROI (red) are averaged apart from spectra in the background region (black). The grey interim area is omitted. Both groupes are averaged separately and plotted in (d). (d) Relevant energy range with characteristic fluorescence energy lines depicted. Five energy intervals are visible, in which the ROI signal is stronger then the background. The grey regions depict intervals of $\Delta_{I}= \pm 0.05 \mathrm{keV}$ around fluorescence lines. The signal within these regions has been integrated for all sample positions and plotted as a function of the position, shown in Fig. 5.11 for four different datasets. The grey regions correspond to the lines of $S_{K \alpha}, K_{K \alpha}$, and $P_{K \alpha}$. The fourth line in the region is assigned to $A l_{K \alpha}$ and $A l_{K \beta}$, see text for discussion. The fifth line is assigned to $K_{K \beta}$ and is therefore redundant to the information from the $K_{K \alpha}$ line. 

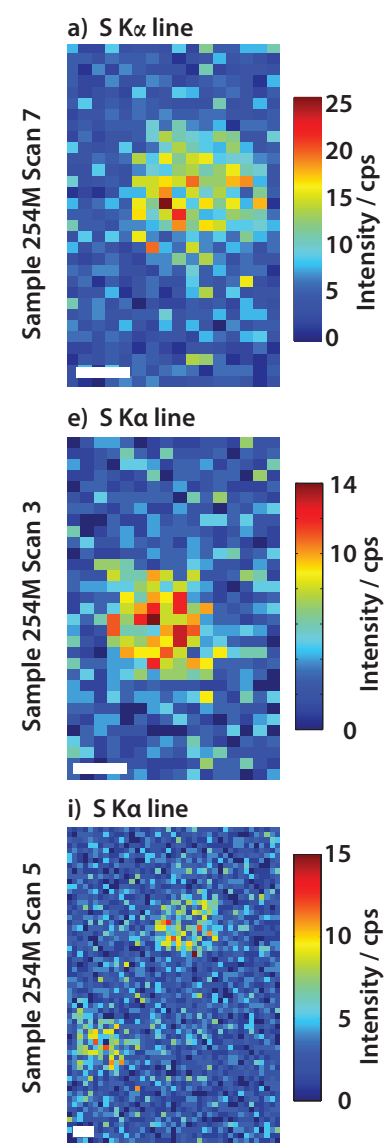

œ m) S Kaline

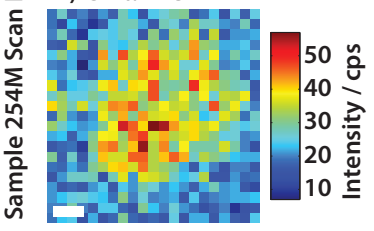

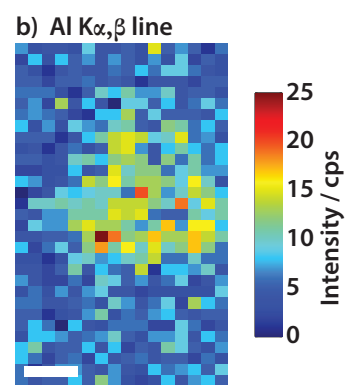
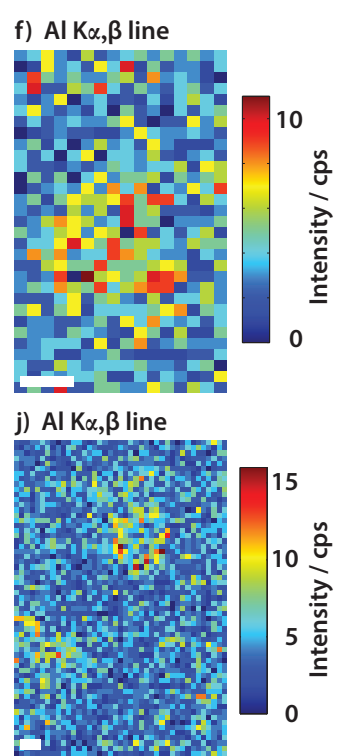

n) Al $K \alpha, \beta$ line

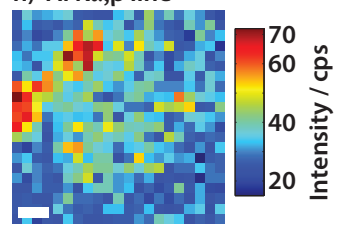

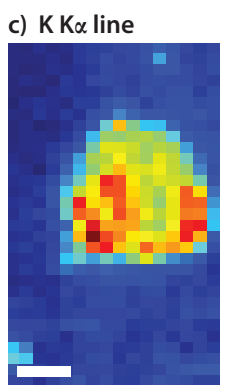

g) KKa line

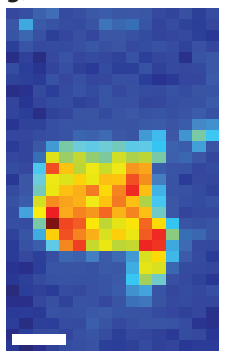

\section{k) K Ka line}

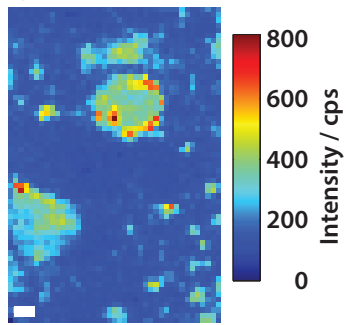

o) KKa line
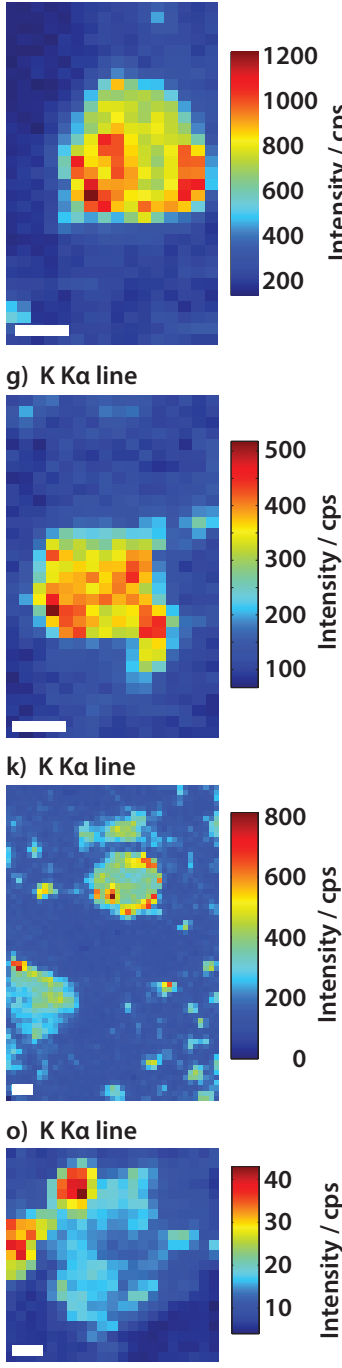

d) $P K \alpha$ line

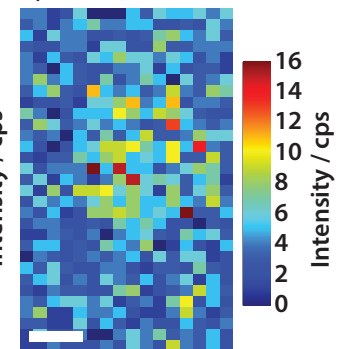

h) P Kaline

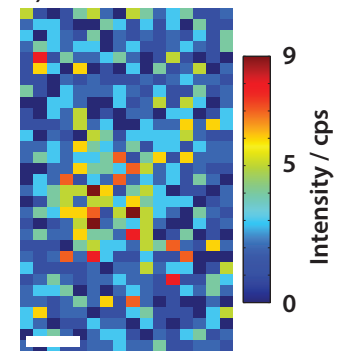

I) P Ka line

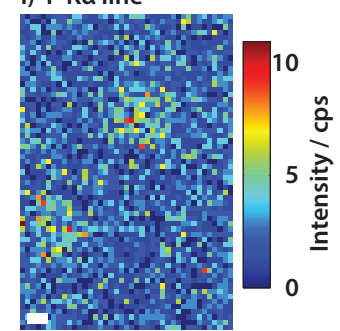

p) P Ka line

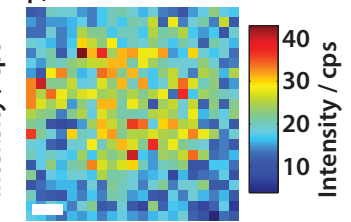

Figure 5.11.: Elemental maps of four energy ranges (columns - see Fig. 5.10) and for four different scanning regions (rows). The cells are best visible in the $K K \alpha$ map, but the other maps show different concentration distributions for other elements (despite the higher noise). Compare, e.g., (o), where the fluorescence signal is most pronounced in two circular regions on the side of the cell, to (m), where the sulfur signal is highest on cell body.

The scans were performed at $1 \mathrm{~s}$ aquisition time per spectrum (i. e., pixel) except for the dataset shown in $(\mathrm{m}-\mathrm{p})$ which was exposed $5 \mathrm{~s}$ per datapoint. All scale bars $2 \mu \mathrm{m}$. 
angle strictly adjusted to $90^{\circ}$ with respect to the primary beam, and better colimation of the detection cone), sufficiently intense spectra should be achievable, so that a batch fitting background modeling can be applied (e.g., standard procedure of PyMCA [71], other techniques are also possible). The fitting approach would allow to quantify the results, unlike in the case of simple channel summation employed in the present analysis. 


\section{Conclusion and Outlook}

We have extended the preparation protocols from freeze-dried to frozen-hydrated samples. Despite a weak contrast of organic material in a water matrix as compared to organic material in air or vacuum, the frozen-hydrated samples allowed SAXS recordings at high signal to noise ratios and revealed localized pronounced orientations in the diffraction patterns.

In our case, an evacuated sample environment as found in soft x-ray setups (due to strong air-absorption, see Fig. A.4) proved not to be necessary, ptychography and nanoSAXS experiments on frozen-hydrated samples could be performed using a commercially available cryogenic jet system at ambient pressure.

The optimization of the cryogenic fixation protocol now allows the preparation at high reproducibility and under well-controlled conditions resulting in fewer defective samples. The cryogenic optical microscope was set up and provides valuable micrographs for sample inspection and for the sample adjustment at beamtimes. Additional polarization contrast could further ease the examining of the samples as ice crystals are birefringent.

Cryo preservation of cells in frozen-hydrated state is widely believed to be the least invasive fixation protocol, our comparison of power-law fits, however, indicates structural differences between the native and plunge-frozen state. This could indicate beam damage happening on time scales much shorter than typical dwell times used $t_{d w e l l}=0.05 \mathrm{~s}$ or structural changes due to the cryo-plunging process. A wide angle detector could help to detect the diffraction signal from crystalline ice $\left(q_{i c e} \in 20 \mathrm{~nm}^{-1} \ldots 60 \mathrm{~nm}^{-1}\right.$ [88]) simultaneously to scanning SAXS recordings. Of particular interest is the correlation with sample thickness, which could indicate either that the cooling rate is too slow for a complete vitrification. Alternatively, cryogenic fixation methods from cryo EM (high pressure freezing or adding cryoprotectants ) are suitable for thicker samples (even tissues) and could be a reference for comparison.

Finally, we even obtain datasets from initially living cells by employing a specialized continuous scanning routine. Compared to previous work [11], the fast migration speed of D. discoideum makes it challenging to capture a cell with a scan region. The structures certainly vary quickly within the cell during one scan, although the cells remain inactive after the first scan. The intensity level in the scattering signal was not sufficient to 
observe the highly anisotropic signal from oriented networks, as found in frozen-hydrated samples. The reduced contrast has two reasons, first, dose efficient scanning requires very short exposures of a sample spot and thus limits the scattering signal accordingly (see Fig. 5.5, $0.05 \mathrm{~s}$ - versus $0.5 \mathrm{~s}$-scan) and second, the channel height of nominally $50 \mathrm{\mu m}$ is 5-10 times larger that the usual thickness of a cell (and its glassy water matrix) of cryo-plunged samples and the contrast is thus further reduced.

In a following experiment, a more intense signal could be achieved by placing a single longer exposure onto a ROI of a (motile) D. discoideum. The ROI could be chosen by parallel observation of the cell with an inline microscope. This would avoid toxic processes (ionization or heating of the cell) within the cell, that may happen on the time scale of several seconds to a few minutes.

Experiments at different preparation states, different x-ray diffractometers, different photon energies, and optics yielded consistent results concerning the diffraction streaks, that occur mainly in the periphery of the cell and are oriented perpendicular to the plasma membrane. These characteristic diffraction streaks are thus found to be an intrinsic structural property of the cell.

The anisotropy parameter $S$ is a valuable tool for a quantification of the orientation in the diffraction pattern of a dataset. The automated algorithms can be used to provide additional information from STXM measurements. Possible modifications are discussed in the corresponding chapter and strongly depend on the datasets and requirements.

The C-shaped appearance of the actomyosin arc and the oriented diffraction pattern along the cell are a strong clue for our interpretation that the actomyosin arc causes these highly oriented diffraction signals. The D. discoideum AX2-LimE-GFP-MyoII-null strain, lacking myosin-II and thus an actomyosin cortex, allows to test this hypothesis. NanoSAXS experiments on these cells could be performed during an upcoming beamtime at GINIX. The streak profiles were read out to extract structural information on the origin of these signals. A simulation of the diffraction patterns of a model filament, constituted from seven hexagonally arranged Gaussian cylinders, was made. The streaklike intensity distribution in the far-field resembles the experimental data, supporting our hypothesis. Yet, the determination of internal structural properties from fibre bundles could not be achieved, as the data show very diverse features and a fitting routine would have too many free parameters.

More information is needed to access the inner structure of the fibre bundles in vitrified (or even living) cells, different approaches are feasible:

By rotating a sample around a fibre bundle in the x-ray focus, many projections provide three dimensional tomographic datasets that should allow further conclusions. 
Our simulation includes the simplest case for an actin fibre bundle, a more sophisticated model for a fibre network will be necessary to extract information from the diffraction data.

Another approach would involve comparison of the scattering curves with data obtained from in vitro actin networks, that are prepared in different constitutions. A model free analysis would be desireable, but does not seem feasible. 


\section{Danksagung}

Meinem Betreuer Prof. Tim Salditt möchte ich sehr herzlich dafür danken, dass er mir diese Arbeit ermöglicht und mich mit fachlicher wie persönlicher Unterstützung ermutigend begleitet hat. Der beständige und verlässlichen Rückhalt durch ihn verdient besondere Anerkennung.

Prof. Jörg Enderlein danke ich für sein Interesse an meinem Thema, sowie die Bereitschaft danken, meine Arbeit als Kobetreuer zu begleiten. Ich danke Prof. Eberhard Bodenschatz und seiner Arbeitsgruppe für die hilfreiche Diskussion über das gemeinsame Forschungsprojekt. Im Rahmen des gemeinsamen SFB-Projektes besteht ein intensiver Austausch mit den Arbeitsgruppen von Prof. Eberhard Bodenschatz und Prof. Jörg Enderlein, für die gegenseitige Unterstützung, den fachlichen Austausch, Anregung und die Möglichkeit, Laboreinrichtung nutzen zu dürfen möchte ich beiden sehr herzlich danken.

Meine Kollegen Robin N. Wilke, Marten Bernhardt, Britta Weinhausen, Valeria Piazza gilt mein Dank für den fachlichen und persönlichen Austausch während der gemeinsamen Zeit.

In gleichem Maße bin ich sowohl für die fachlichen Anregungen und Diskussionen als auch für den exzellenten persönlichen Kontakt zu meinen Kollegen aus der Arbeitsgruppe von Herrn Prof. Bodenschatz, Christoph Blum und Marco Tarantola, sowie Christian Westendorf und Azam Gholami dankbar .

Die gemeinsame Nutzung von Messzeiten und die gegenseitige Unterstützung währenddessen habe ich als sehr vorteilhaft erlebt und danke hierfür Prof. Sarah Köster und Britta Weinhausen. Ferner bedanke ich mich bei vielen Leuten, die die Experimente an der GINIX erst ermöglicht haben, hierbei sind insbesondere Markus Osterhoff, Martin Krenkel, Matthias Bartels, Bastian Hartmann, Sebastian Kalbfleisch und Britta Weinhausen zu nennen. Den Kollegen in der Verwaltung von Institut und den SFBs 937 und 755, Sabine Balder, Christina Emser, Kerstin Pluschke, Eva Hetzel, Kerstin Grüny, Rebecca Benez und Doris Thies gilt mein Dank für die stille und immer aufmerksame wie hilfsbereite Unterstützung bei zahlreichen Vorgängen.

Meinen Kollegen in den Werkstätten, Peter Nieschalk und Carsten Wulf gilt mein Dank, da sie viele Experimente mit Ihrer Arbeit überhaupt erst ermöglicht haben. In den 
Laboren bin ich besonders dankbar für die Unterstützung von Jochen Herbst, Thorsten Gronemann und Julia Scherber. Außerdem danke ich den Mitarbeiterinnen im Zellkulturlabor der Arbeitsgruppe Bodenschatz, Katharina Schneider, Tina Althaus und Marion Kunze für die großartige und des Öfteren kurzfristige Vorbereitung von Zellen, sowie Ihre Bereitschaft mir viele Fragen zum Umgang mit Zellen zu beantworten.

Die Kollegen in unserem „Ingenieurbüro“, Bastian Hartmann und Mike Kanbach haben mir durch Rat und Tat in viele Fragen weitergeholfen, wofür Ihnen ein ganz besonderer Dank gebührt.

Jan Goeman ist durch seine kompetente, schnelle und immer freundliche Unterstützung eine unverzichtbare Säule vieler Projekte geworden und sei herzlich für seinen Einsatz bedankt.

Die Messzeiten an P10 (DESY), ID13 (ESRF) und cSAXS (SLS) sind erst möglich durch die Unterstützung der Beamline-Wissenschaftlerinnen und -Wissenschaftler, dafür möchte ich den folgenden Personen ganz besonders danken: Michael Sprung, Fabian Westermeier, Alexey Zozulya und Sergej Bondarenko für die P10; Michael Burghammer, Michael Sztucki und Emanuela Dicola für die ID13; Ana Diaz, Xavier Donath und Andreas Menzel für die cSAXS.

Für Ihre Hilfe beim Korrekturlesen und Anregungen danke ich Katharina Echternkamp, Marco Tarantola, Markus Osterhoff und Horst Hoffmann. Die Kollegen im Institut tragen zu einer freundlichen und offenen Athmosphäre bei, für die ich mich beim Institut bedanken möchte. Insbesondere meinen aktuellen und ehemaligen Büronachbarn gilt dieser Dank.

Dem Team der Hautklinik am Universitätsklinikum Göttingen bin ich für die Hilfestellung und Behandlung, die mir während der Doktorzeit zuteil wurde, in großer Dankbarkeit verbunden - ohne sie wäre mir die Doktorarbeit kaum möglich gewesen.

Meinen Eltern und Familie danke ich für die vielseitige Unterstützung auf meinem Weg hierher und den Glauben an mich. Gleiches gilt für meine Freundin Katharina, die in der letzten Zeit viel Verständnis für mich aufgebracht, mich unterstützt und ermuntert hat. Ihr und ihrer Familie möchte ich für ihre Unterstützung herzlich danken.

Diese Arbeit wurde unterstützt durch den Sonderforschungsbereich 937 „Kollektives Verhalten weicher und biologischer Materie“ der DFG, Projekt A11. Die Synchrotronstrahlungseinrichtungen DESY Photon Science, ESRF und SLS seien für die Strahlzeit bedankt. 


\section{A. Additional Graphs and Images}

\section{A.1. Fluorescence Data}

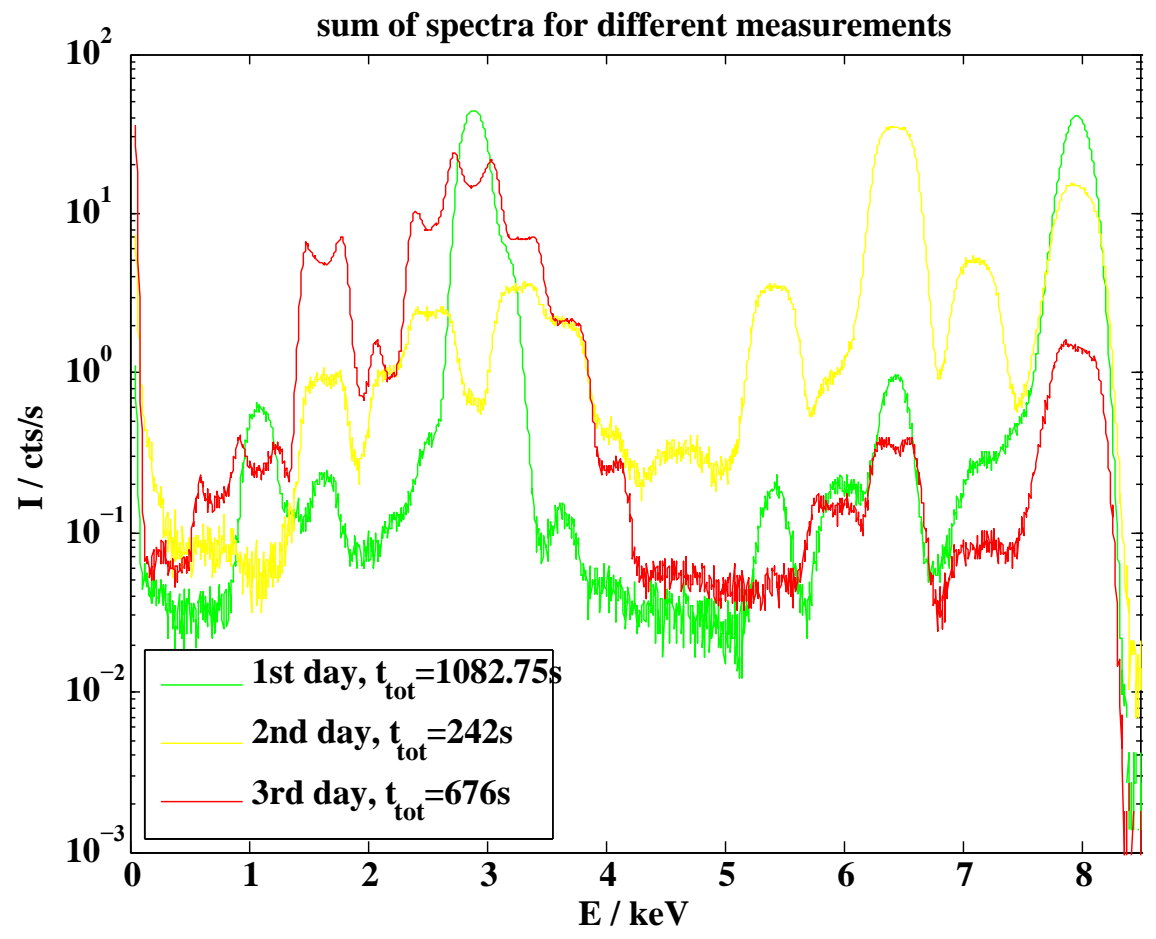

Figure A.1.: Development of fluorescence spectra over several days during a beamtime at GINIX, P10. The curves show a slow degradation of the peakshape over three days. Saturation effects did not cause this behaviour, in a second set of aquisitions the detector was moved further away from the sample and an additional absorber was placed in the primary beam, without any influence on the peak shape. The curves were obtained from scans by averaging and normalization with the aquisition time. 

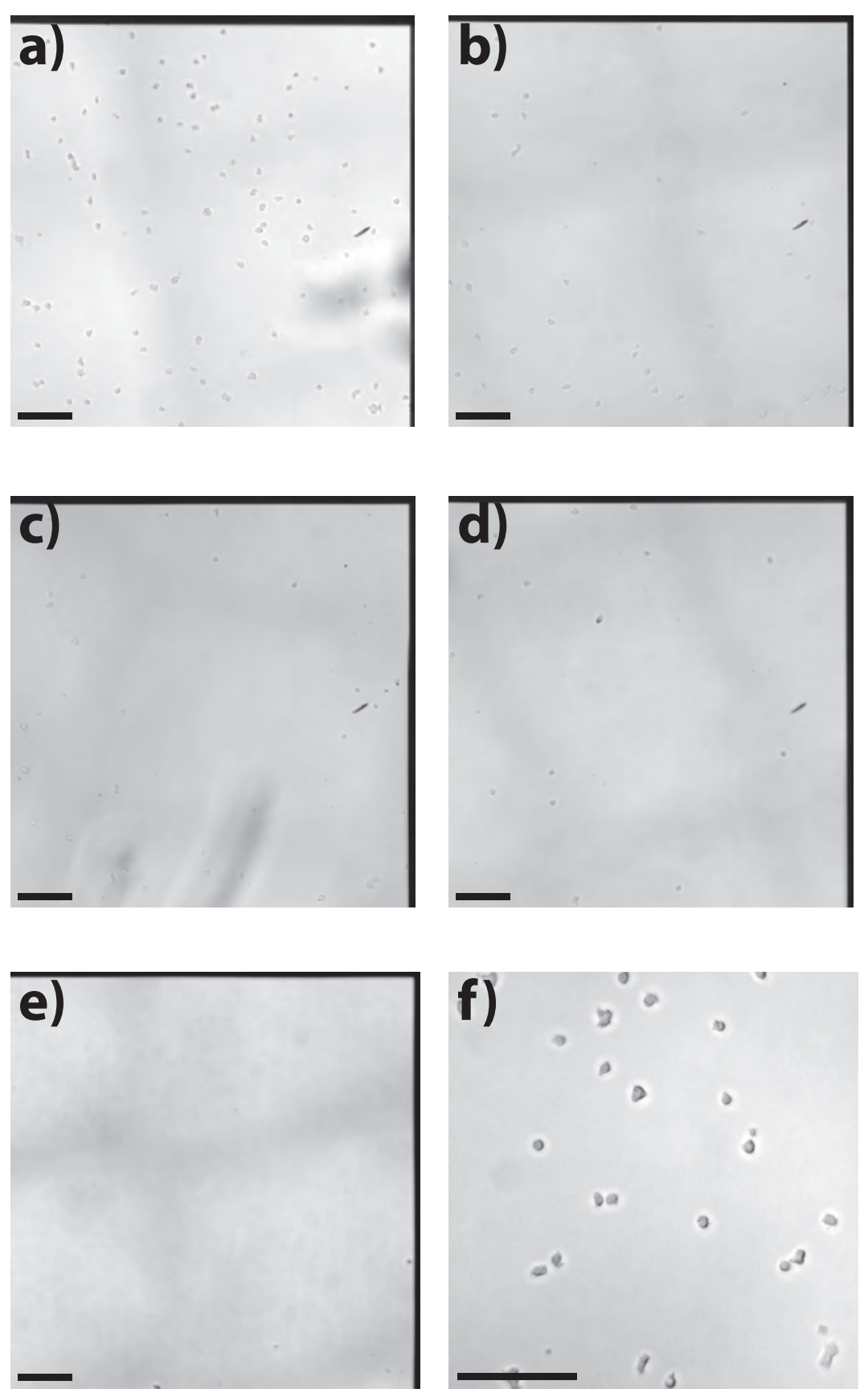

Figure A.2.: Development of a sample of $D$. discoideum on a silicon nitride membrane during the preparation of frozen-hydrated sample. The images show a quarter of a silicon nitride membrane, imaged after every step of the preparation protocol for frozenhydrated cells, videlicet (a) after adhering, (b) after taking the substrate out of the buffer, (c) after mounting the substrate in the gridplunger, (d) after gently whashing the substrate in the gridplunger, and (e) after blotting the sample in the gridplunger. (f) shows an inset of the sample after adhering in a greater magnification than (a) to illustrate the motile condition of the cells. The scale bars equate $100 \mu \mathrm{m}$. 

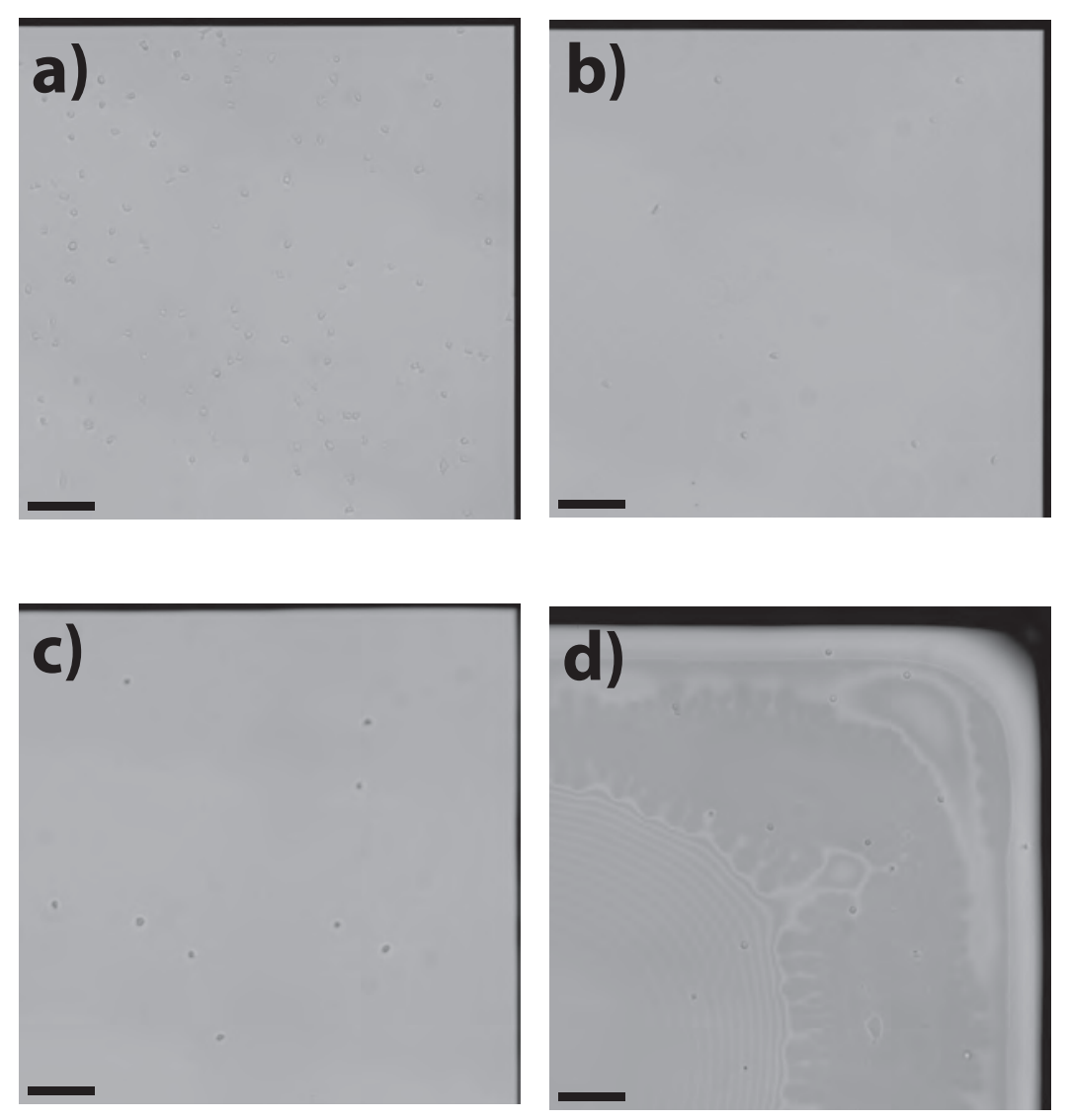

\section{e)}

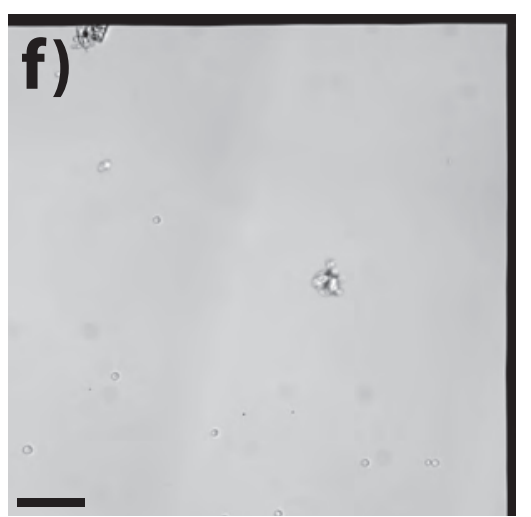

Figure A.3.: Comparison of three samples of $D$. discoideum on silicon nitride membranes $(\mathbf{a}, \mathbf{c}, \mathbf{e})$ before the first and $(\mathbf{b}, \mathbf{d}, \mathbf{f})$ before the last step of the preparation protocol for frozen-hydrated samples. The samples (c-f) show very few cells initially, for cellular imaging, these would not have been considered for synchrotron experiments. The cellular density found on the samples in (a-b) and also in Fig. A.2, however, are suitable for a scanning diffraction exeriment. 


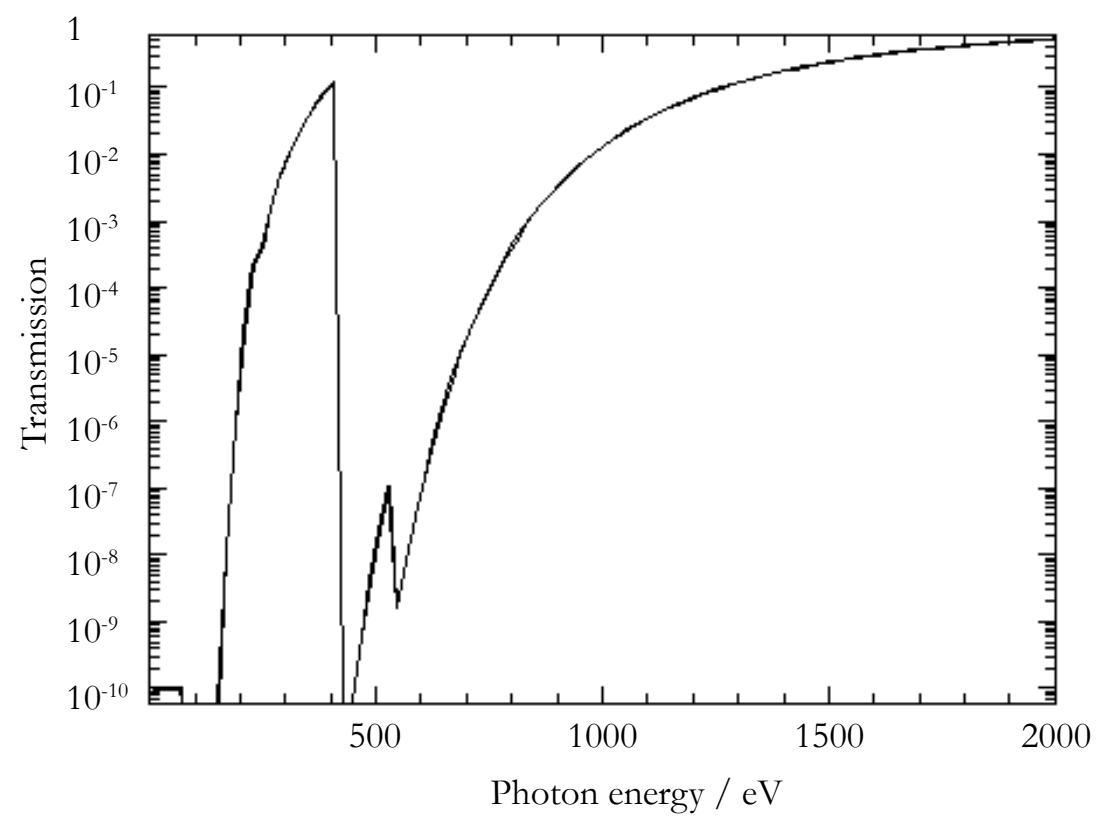

Figure A.4.: X-ray transmission curve of $1 \mathrm{~cm}$ air for $E_{P h} \in 10 \mathrm{eV} \ldots 2000 \mathrm{eV}$. the transmission is small for photon energies of $1000 \mathrm{eV}$ and below, except for one peak (due to absorption edges) at $400 \mathrm{eV}$, which explains the necessity to work in vacuum with soft x-ray experiments.

Graphic adapted from "Centre for x-ray optics", http://henke.lbl.gov/ optical_constants/gastrn2.html, theoretical value for air under ambient pressure at $295 \mathrm{~K}$ and a given path length of $0.1 \mathrm{~cm}$, last accessed 5.1.2015. Reference: [89] 


\section{B. Simulated Fibre Diffraction and Reciprocal Space Transformation}

In the simulation presented in Sec. 3 of the main article, the necessary transformation of the reciprocal space associated with the fibre bundles to that of the laboratory frame is handled as follows. We first consider a fibre axis which is inclined at an angle $\vartheta$ with respect to the optical axis. Hence, for $\vartheta=0^{\circ}$ the diffraction pattern would correspond to a cut through the azimuthal plane in reciprocal space $Q_{X}, Q_{Y}$ reflecting the twodimensional structure factor $S F\left(Q_{x}, Q_{y}\right)$ calculated from the filament positions $\mathbf{R}_{\mathbf{j}}$. This could correspond to an ordered $2 \mathrm{~d}$ liquid or a crystalline arrangement, such as a $2 \mathrm{~d}$ cubic or hexagonal pattern, depending on the physical situation. For $\vartheta=90^{\circ}$, the fibre axis is perpendicular to the optical axis along a radial line on the detector perpendicular to the streak and forming an angle $\chi$ with respect to the $q_{z}$ axis. Along with a rotation around the fibre axis $\varphi$ the fibre bundles orientation is fully specified by the parameters $\chi, \vartheta, \varphi$.

We first apply a rotation $\mathcal{R}_{\chi}^{x}$ of the detector frame around the optical axis $\left(x, q_{x}\right)$ in order to obtain the detector axis $q^{\prime} z$ intersecting with the plane suspended by the fibre axis and the optical axis, i.e.

$$
\left(\begin{array}{c}
q_{y}^{\prime} \\
q_{z}^{\prime}
\end{array}\right)=\left(\begin{array}{cc}
\cos \chi & \sin \chi \\
-\sin \chi & \cos \chi
\end{array}\right)\left(\begin{array}{l}
q_{y} \\
q_{z}
\end{array}\right)
$$

From $\left(q^{\prime} y, q^{\prime} z\right)$, the components of the momentum transfer vertical and perpendicular to the fibre axis are easily obtained according to

$$
\begin{aligned}
Q_{z} & =q_{z}^{\prime} \sin \vartheta \\
Q_{\|} & =\left(q^{\prime}{ }_{y}^{2}+q_{z}^{2} \cos ^{2} \vartheta\right)^{0.5}
\end{aligned}
$$

and finally by rotation $\mathcal{R}_{\phi}^{Z}$ around the fibre axis the components in the fibre coordinate system required for the structure factor

$$
\begin{aligned}
Q_{x} & =Q_{\|} \cos \phi \\
Q_{y} & =Q_{\|} \sin \phi
\end{aligned}
$$




\section{Matlab Scripts}

\section{C.0.1. Streak Finder Algorithm}

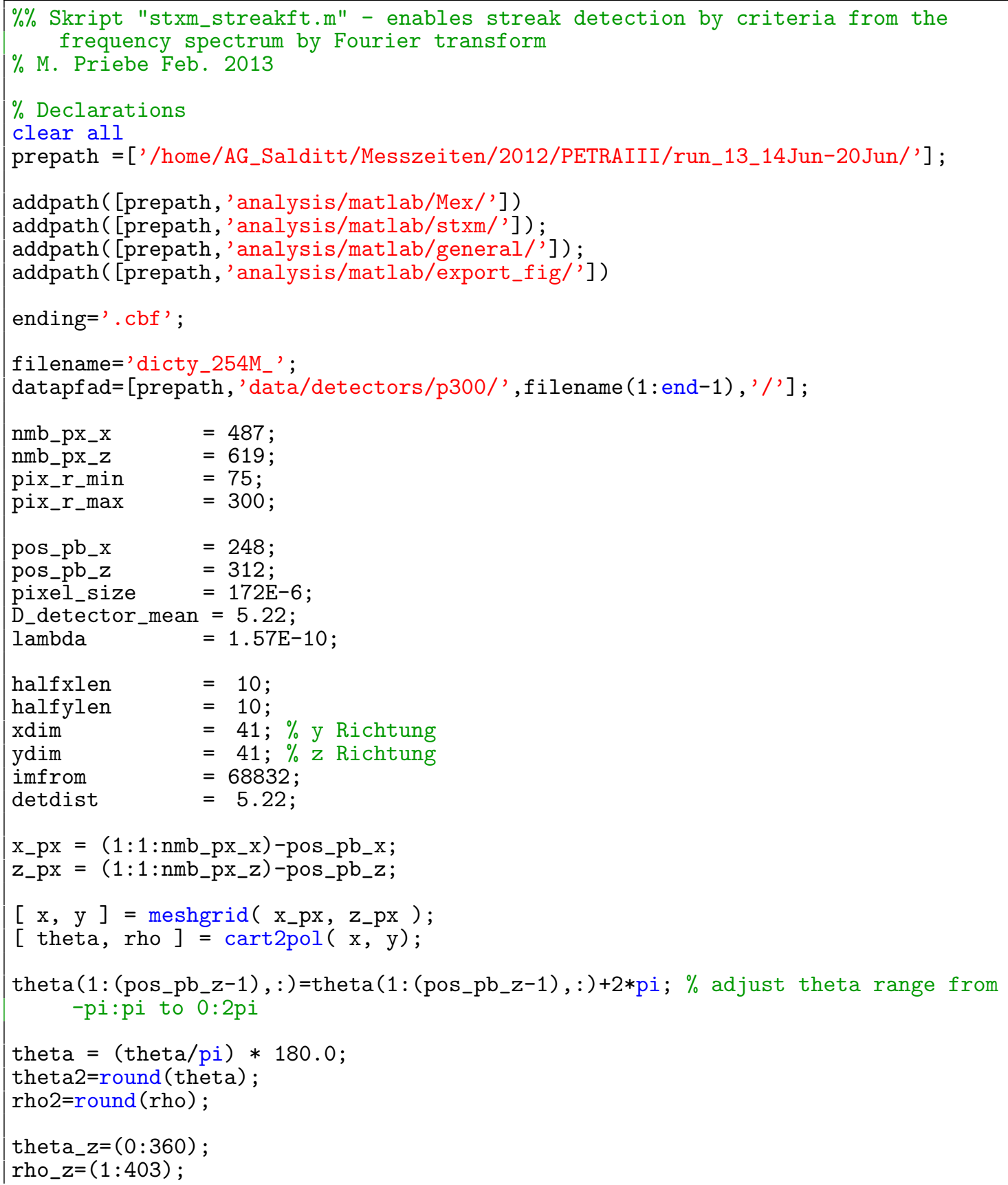




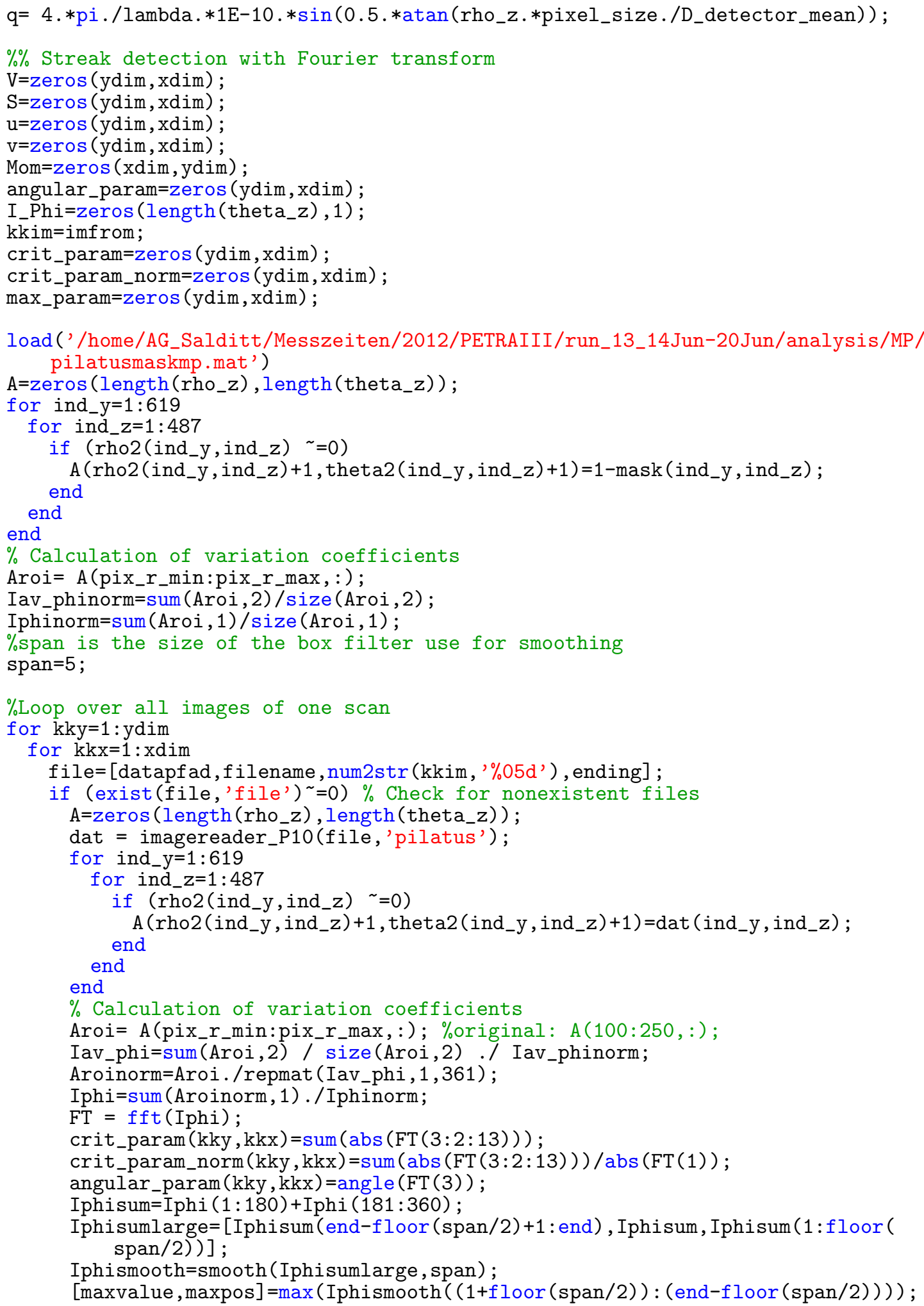




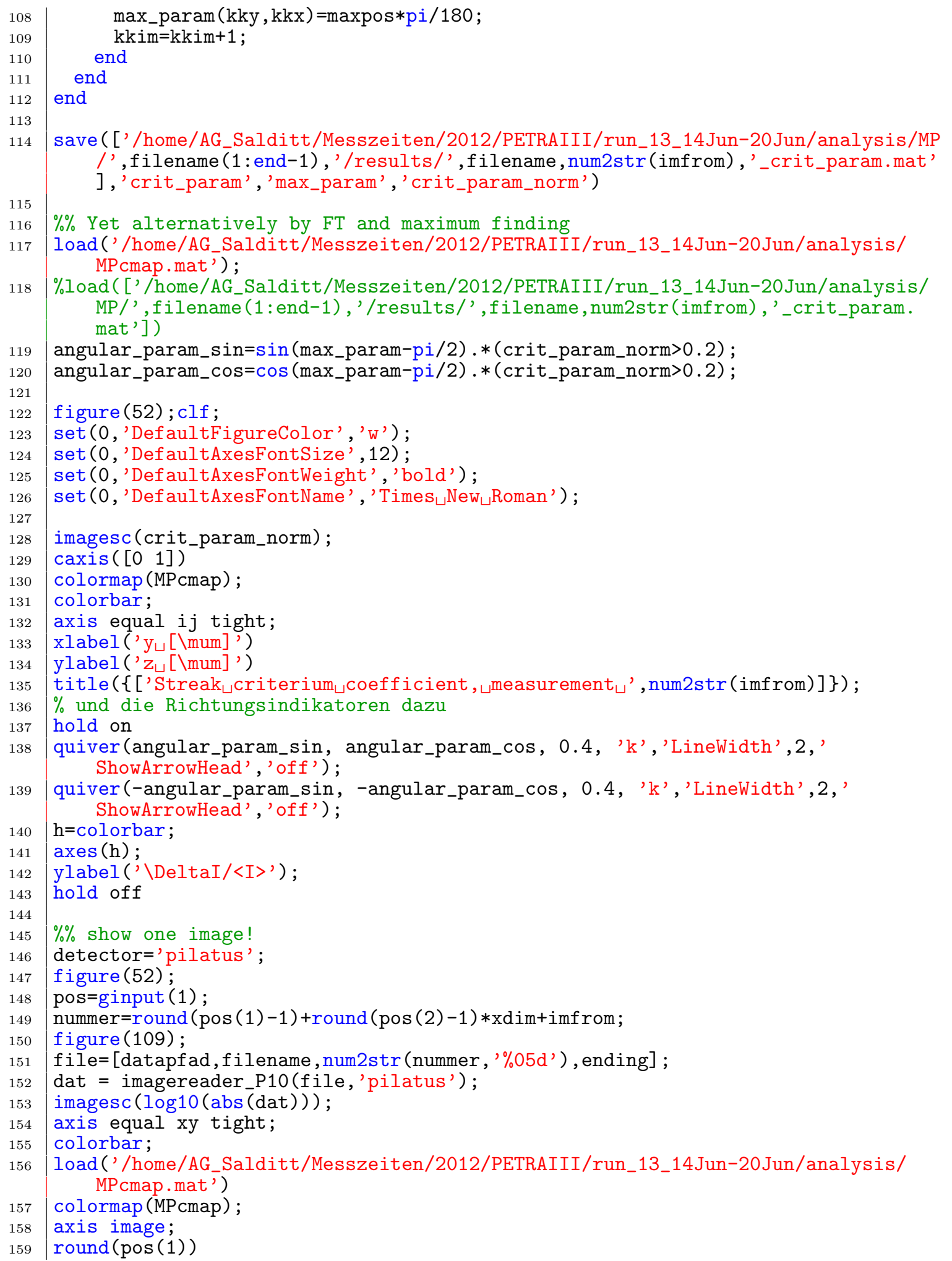




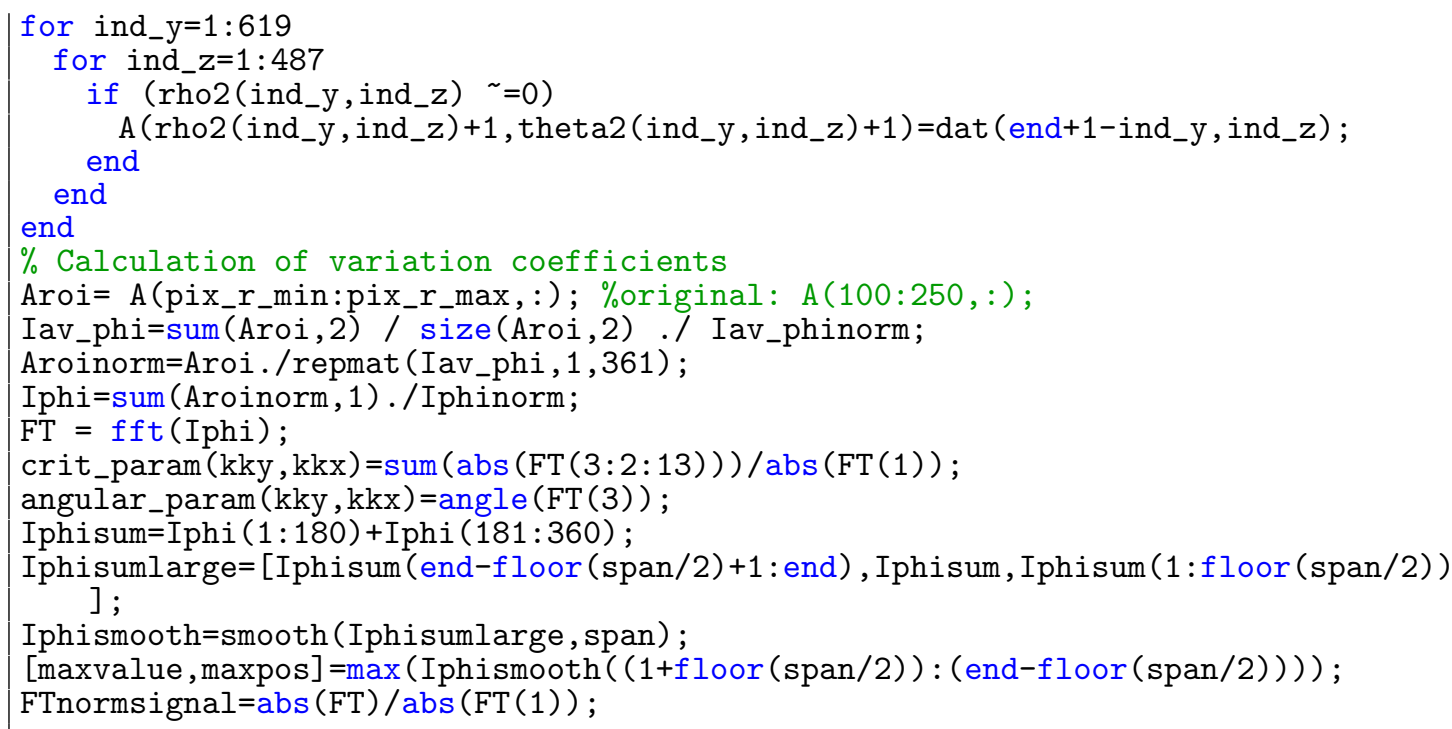




\section{C.0.2. Fluorescence Mapping}

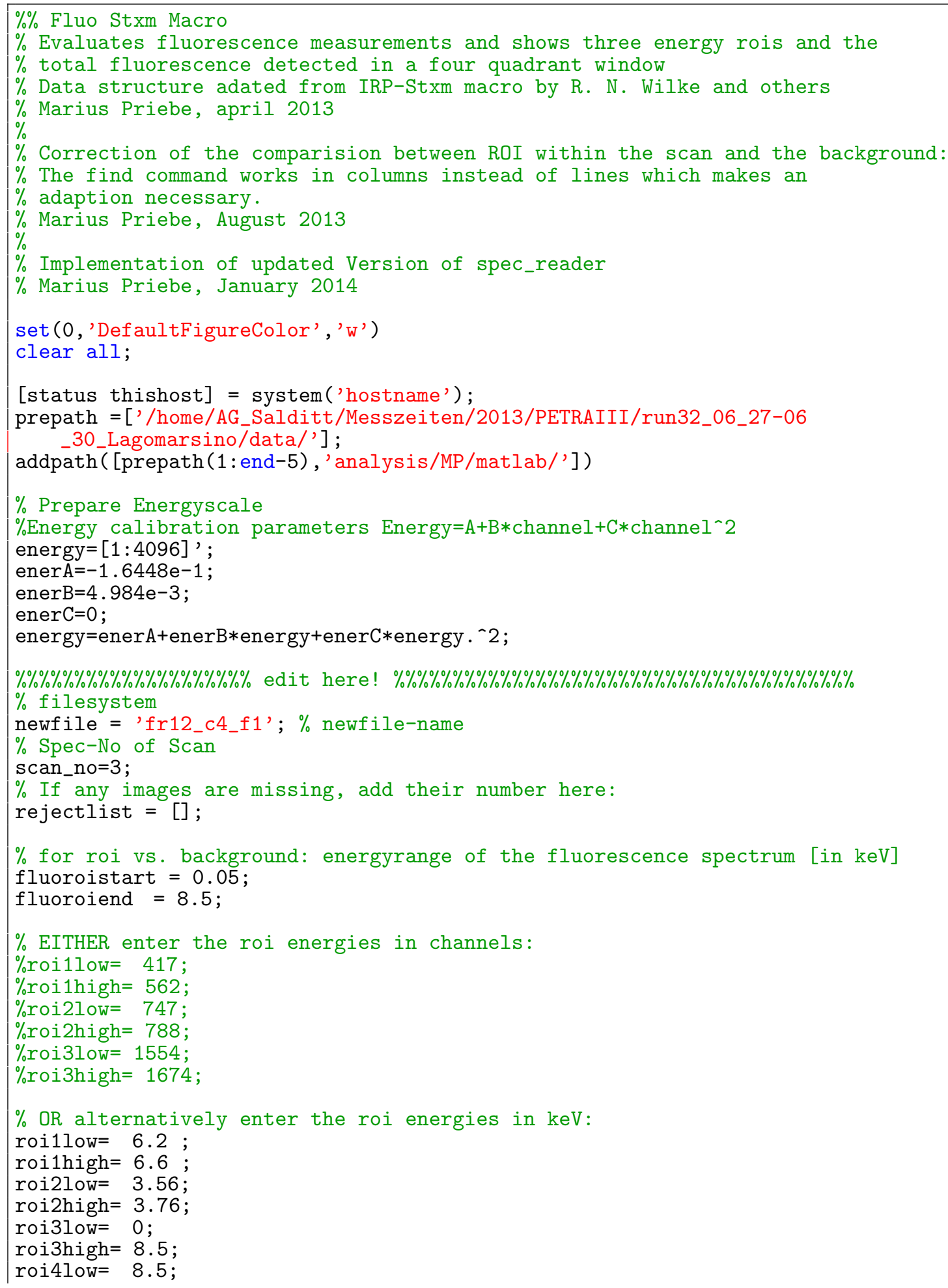




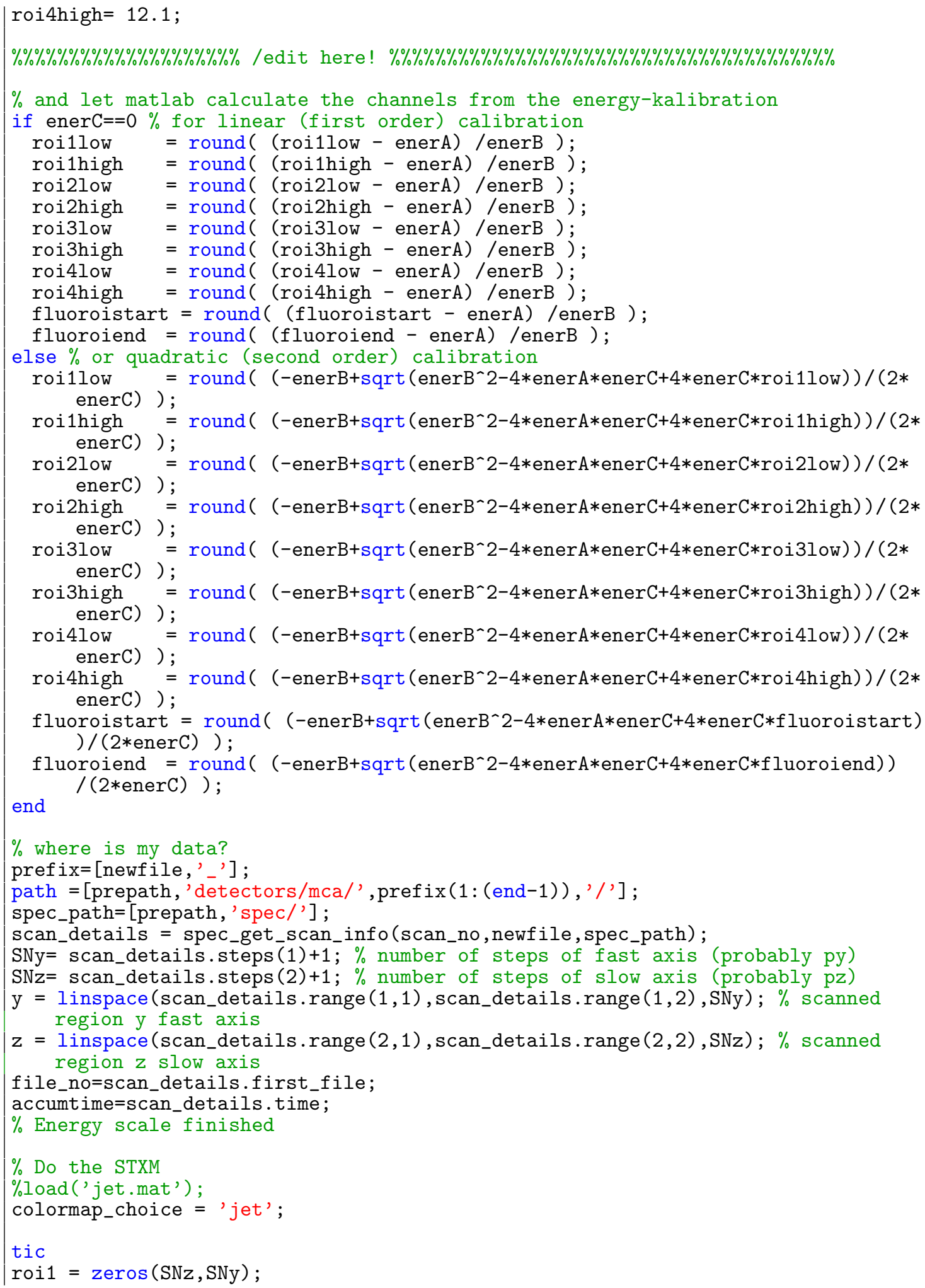




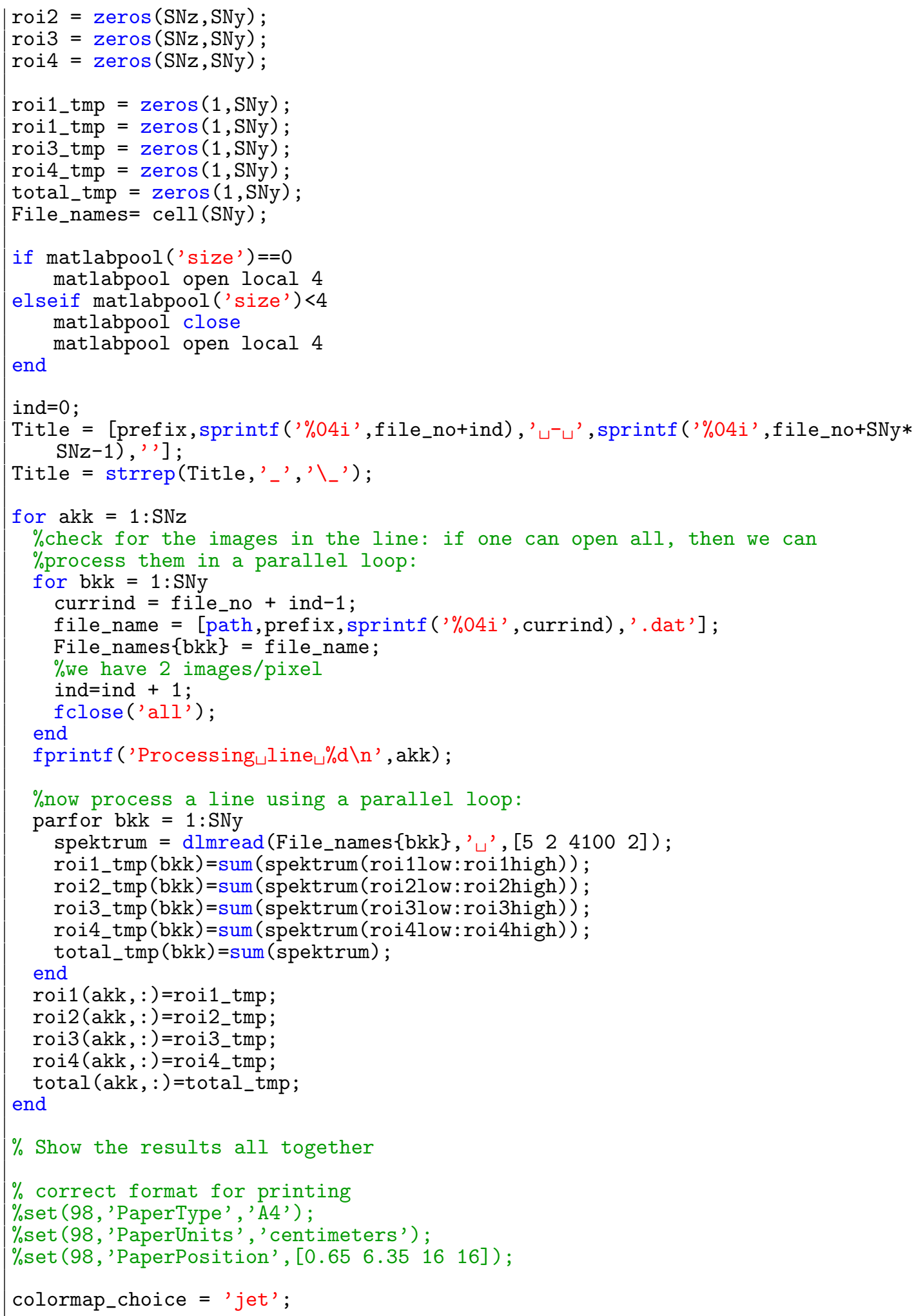




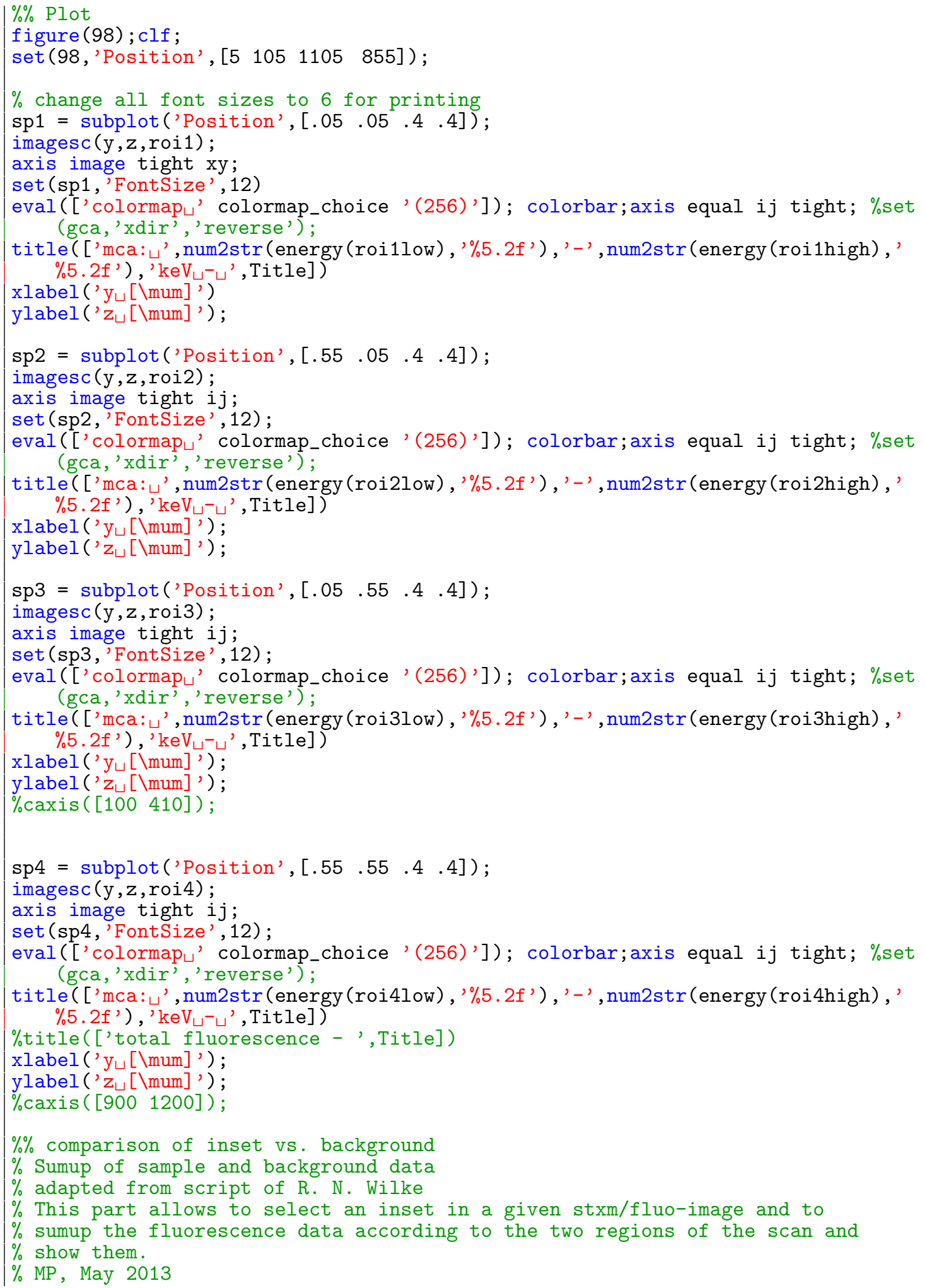


$\%$ draw figure to create mask

close all;

figure (1)

imagesc(y,z,roi2); \% select different roi here, if necessary

colormap gray (256);

colorbar;

axis equal tight;

xlabel ('fast $\sqcup$ axis')

ylabel ('slow

$\%$ define sample roi

mask $=$ createMask (impoly);

mask $=\operatorname{double}($ imfill $($ mask, 'holes' $))$;

$\%$ create buffer region, take rest as background

se $=\operatorname{strel}(' d i s k ', 2)$;

notmask $=\sim$ imdilate $($ mask, se $)$;

$\%$ now get linear indices;

lin_ind_cell $=$ find $(f l i p u d(\operatorname{rot} 90($ mask $)))$;

lin_ind_no_cell $=f$ ind $(f l i p u d(\operatorname{rot} 90($ notmask $)))$;

$\%$ Iin_ind_no_cell = find ( $\left.{ }^{\text {mask }}\right)$;

$\%$ Do the STXM

ind $=0$;

filenames $=\operatorname{cell}(1, \mathrm{SNz} * \mathrm{SNy})$;

for $a k k=1: \mathrm{SNz}$

for bkk $=1: \mathrm{SNy}$

currind = file_no + ind;

file_name $=$ [path,prefix, sprintf ('\%04i', currind), '.dat'] ;

$\%$ fprintf (file_name)

filenames $(1, \operatorname{SNy} *(a k k-1)+b k k)=\left\{f i l e \_n a m e\right\} ;$

$\%$ we have 2 images/pixel

ind $=$ ind +1 ; end

end

$\%$ fprintf (['\n1st file: ', filenames $\{1,1,1\}$ (length (data_dir) +1 :end), '\n']);

$\%$ fprintf (['\nlast file: ',filenames $\{1$, end, end\}(length(data_dir) +1 : end), ' $\backslash$ n'] );

$\%$ sum all the diffraction patterns

$\%$ load all the diffraction patterns

filename_cell = filenames(lin_ind_cell);

filename_no_cell = filenames (Iin_ind_no_cell);

if matlabpool ('size') $==0$

matlabpool open local 4

elseif matlabpool ('size') $<4$

matlabpool close

end

matlabpool open local 4

data_cell=zeros (4096, length (lin_ind_cell));

data_no_cell =zeros $(4096$, length $(1$ in_ind_no_cell));

parfor akk=1:length(filename_cell)

data_cell (:,akk) =dlmread (filename_cell\{akk\}, ' ', , [5 24100 2] );

end

parfor akk=1:length(filename_no_cell)

data_no_cell (:,akk) =dlmread (filename_no_cell\{akk\}, ' ', , [5 24100 2] ); 


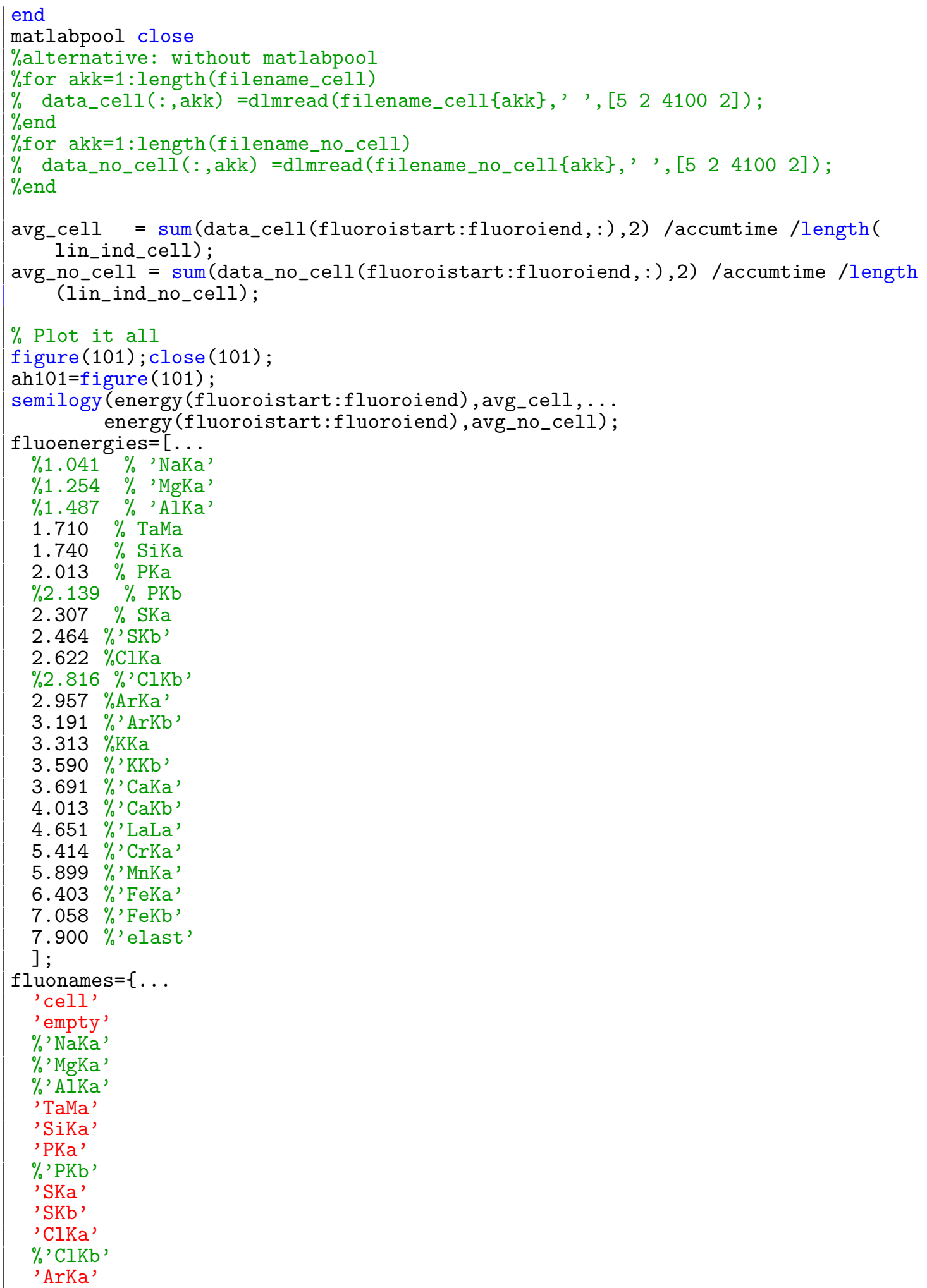




\footnotetext{
'ArKb'

' $\mathrm{KKa}$ '

' $\mathrm{KKb}$ '

'CaKa'

'CaKb'

'LaLa'

'CrKa'

'MnKa'

'FeKa'

'FeKb'

'elast'

\} ;
}

for ener=fluoenergies

line([ener ener],ylim, 'LineStyle', '- .' , 'LineWidth', 1.5) end

$\% \operatorname{tah} 101$ = addtxaxis (gca, ' $\mathrm{x}$ ', fluoenergies, 'fluorescence lines',fluonames); legend (fluonames, 'location', 'bestoutside')

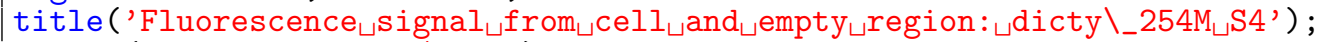

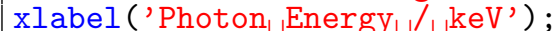

ylabel ('Intensity $\sqcup / \sqcup \mathrm{cps}$ ') ;

fluonames $=\{\ldots$

'data'

$\%$ 'NaKa'

$\%$ 'MgKa'

$\%$ 'AlKa'

$\%$ 'TaMa'

'PKa'

\%' $\mathrm{PKb}$ '

'SKa'

'SKb'

'ClKa'

$\%$ ' $\mathrm{ClKb}$ '

'ArKa'

'ArKb'

' $\mathrm{KKa}$ '

' $\mathrm{KKb}$ '

'CaKa'

'CaKb'

'LaLa'

'CrKa'

'MnKa'

'FeKa'

'FeKb'

'elast'

\};

ah102=figure (102); clf;

\%semilogy (energy (fluoroistart:fluoroiend), abs (avg_cell-avg_no_cell));

$\%$ semilogy (energy (fluoroistart:fluoroiend), (avg_cell-avg_no_cell), 'black',...

\%energy (fluoroistart:fluoroiend), (-avg_cell+avg_no_cell), 'red');

plot (energy (fluoroistart:fluoroiend), (avg_cell-avg_no_cell), 'black');

$\% \operatorname{tah} 102$ = addtxaxis (gca, 'x',fluoenergies, 'fluorescence lines',fluonames);

for ener=fluoenergies

line([ener ener],ylim, 'LineStyle', '- .' , 'LineWidth',1.5) end

title('Difference $\sqcup$ fluorescence $\sqcup$ signal $\sqcup 0 n_{\sqcup} c e l l_{\sqcup m i n u} s_{\sqcup} e m p t y \sqcup$ signal: $\sqcup$ dicty $\backslash 254 M_{\sqcup}$ S4');

xlabel ('Photon $\sqcup$ Energy $/\lrcorner k e V ')$;

ylabel ('Intensity $/ \sqcup \mathrm{cps}$ ') ; 
397 legend(fluonames, 'location', 'bestoutside') 


\section{Operations Manuals}

\section{D.1. Cryo Wiki}

\section{D.1.1. Cryostream}

\section{Install Cryo}

This is a manual for trained users! Please seek the assistance of someone experienced with the setup before trying it on your own!

\section{You need...}

\section{... for the cryo setup:}

1. Two long tubings (controller to head)

2. Two hort tubings (controller to pump)

3. One cable (controller to head)

4. Power cord

5. Controller

6. Pump

7. Cryo head

8. LN2 supply dewar

\section{... for the LN2 refill system:}

1. Refill controller

2. 2 BNC Cables

3. Oscillator

4. Level sensor (Please handle with care!)

\section{Hardware Setup}

\section{Install the cryo stream}

1. Bring the supply dewar to its dedicated place.

2. Install the dewar head on the supply dewar.

3. Install cryo head.

4. Insert the LN2-supply pipe of the cryo head very carefully into the supply dewar. Gently tighten the sealing with your hand (no tools required!!!)!

5. If necessary put tin foil shielding or a hose around the over pressure outlet of the dewar head, if it points at components sensitive to cold nitrogen gas or nitrogen spill; a strong gas stream will exit there. 
6. Connect all cables and tubings:

a) Two tubes from controller to cryo head according to the labels (connector and tube end are named identically)

b) Two short tubes from the controller to the pump

c) Long cable from controller to cryo head

d) Power supplies: controller to socket, pump to the socket of the controller

\section{Install LN2 refill system}

1. Connect the refill tube to the supply dewar of the cryostream.

2. Connect the refill tube to the tube cross of the refill system, use the connector of the end with the magnetic valve.

3. Connect the tube cross to the refill dewar using an aluminium sealing for KF40 flanches; make sure, the tube cross is well aligned with the exit pipe of the refill dewar.

4. Carefully put the level sensor into its port of the dewar head, gently tighten the screw.

5. Connect the refill controller to the level sensor using one BNC cable, followed by the oscillator and another BNC cable; mind the orientation of the oscillator (indicated).

6. Connect the power supply of the refill controller. All settings should be correct from the beginning. If you have questions, please contact M. Priebe.

7. Bring valve switch in middle position "closed".

\section{Start Cryo}

1. Fill the supply dewar with LN2.

a) Switch on the refill controller.

b) Check the following set points with the turning knob: HI, LO, A, B. To do so, put turning knob in desired position and click - do not hold - the neighbouring switch to "raise" or "lower". Note: Holding the knob will alter the set points! Highly recommended: $\mathrm{LO}=20.8, \mathrm{~B}=24.9, \mathrm{~A}=97.5, \mathrm{HI}=99.7$

c) Turn knob to "silent".

d) Carefully open the exit valve of the refill dewar.

e) Bring switch in "auto" position; stay with the system permanently while the level is still below "LO" level.

f) As soon as LN2 level reaches LO, bring turn knob into position HI, LO, A, or B.

g) Refill process should end automatically when $99 \%$ level is reached.

2. Open dry nitrogen and regulate to $101 / \mathrm{min}$.

3. Switch on cryo on main switch.

4. Wait for self check to finish; when "hours .... serial ..." shows, press display.

5. Set desired temperature.

6. Press start.

7. Wait for final temperature to be reached (cooling to 100K might take 20-30 Minutes). 


\section{Stop Cryo}

Never simply press "Stop" or switch off the system! It has to be heated up before beeing halted!

1. Press program button.

2. Navigate with turn knob to entry "end" and confirm with "enter".

3. Set heating rate to $360 \mathrm{~K} / \mathrm{h}$ with turn knob, confirm with "enter".

4. Press "start" to activate program.

5. The cryo will halt as soon as $300 \mathrm{~K}$ are reached.

6. After this has happened, the controller can be switched off.

\section{D.1.2. Automatic Dewar Refill System}

Short Operation Manual for the Automatic Dewar Refill System (Version 1.0, MP 2012/10/10)

\section{General Remarks}

Simply leave the system running and check the level of the refill dewar daily. In operation mode:

- The switch at the control panel is put to "AUTO".

- The pressure relief valve of the cross is closed (between the exit valve of the refill dewar and the magnetic valve there is a pipe with a red valve).

- The exit valve of the dewar is open.

Write down when there is a refill, so that you can estimate when refill dewars has to be replaced.

\section{How to Change the Refill Dewar}

1. Set the magnetic valve to close with the switch on the control panel.

2. Close the exit valve of the refill dewar.

3. Carefully open the pressure relief valve of the cross (between the exit valve of the refill dewar and the magnetic valve there is a pipe with a red valve).

4. Open the KF-flange which is situated directly behind the dewar exit valve. Discard the aluminium sealing, use a new one every time!

5. Take away empty dewar, position new one.

6. Connect the KF-flange using a new Al-sealing. Keep in mind to use the outlet marked "FL" (fluid), not "GAS".

7. Close the pressure relief valve.

8. Carefully open the exit valve of the dewar. In case of any leaks, close it again and seal them.

9. Put the switch on the front panel of the controller back to "AUTO".

The system should now be working again. 


\section{Further Information}

The refill system has a sensor that measures the liquid nitrogen level inside the cryostream dewar. The level is displayed in centimetres, percent or inches, to be selected by the switch on the front panel of the controller. Four set points are defined to guarantee proper working, namely "Low", "B", "A", and "Hi". If the level sinks below "Low", an acoustic alarm will sound, and simultaneously the corresponding red control light shows on the control panel. The set point "B" must be above "Low" and activates the refill process (if the valve switch is set to "AUTO"). The valve will close again, as soon as the level reaches the height set as "A". Accordingly "A" has to be above "B". The "High" set point is above "A" and will trigger an acoustic alarm. Thus, if there is an acoustic alarm or you see one of the lamps "Low" or "High" shining red, please take the following action: "Low" alarm: Check for closed valves or an empty dewar. "High" alarm: immediately close hand valve of the refill dewar. Then check if it is possible to close the magnetic valve with the switch (probably stuck).

\section{Set Points Values}

Please DO NOT change these without further consultation with Marius Priebe!

\begin{tabular}{lrr}
$\mathrm{Hi}$ & $48.1 \mathrm{~cm}$ & $99.7 \%$ \\
\hline $\mathrm{A}$ & $47 \mathrm{~cm}$ & $97.5 \%$ \\
\hline $\mathrm{B}$ & $12 \mathrm{~cm}$ & $24.9 \%$ \\
\hline Low & $10 \mathrm{~cm}$ & $20.8 \%$
\end{tabular}




\title{
D.2. User Manual for Fluorescence Detector Ketek with XIA MCA
}

\section{D.2.1. Data}

Data is stored in the canonic detectors folder

/data/2013A/20130626/detectors/mca/<newrun>/

\section{Spec Commands}

\author{
mcaon activates mca from next scan/ct on \\ mcaoff deactivates mca from next scan/ct on \\ mcasetroi defines energy rois calculated for every acquisition (mcaROI0 - mcaROI2) \\ usage mcasetroi counter_mne low_channel high_channel \\ e.g., mcasetroi mcaROI1 10100
}

\section{D.2.2. Setup of the Ketek Detector at Ginix-for Trained Users}

Please do not use the steps and commands below, unless you really know what you are doing! Do not hesitate to seek assistance!

Implementation by S. Kalbfleisch and M. Priebe

\section{Needed:}

- Ketek fluorescence detector head (in Ketek case)

- Ketek supply and preamp box (in Ketek case)

- Cables in Ketek case: 1 USB, 1 coax cable BNC-Lemo, preamp cable Lemo-Lemo

- XIA Mercury multi channel analyser (in big black plastic box)

- Dell mini PC with Epics

- Cables: 3 IEC power connector cords "Kaltgeraetesteckerkabel", 1 network cable

\section{D.2.3. Connect...}

- Big Lemo cable from detector head to Ketek supply and preamp box

- BNC-Lemo calbe from detector head to XIA MCA connector "Input" on rear side

- USB from rear side connector of XIA Mercury to PC

- Power supply to XIA Mercury, Ketek supply, Ketek preamp box, and to PC

- Network cable from PC to control subnet of a switch

\section{Startup}

1. Switch on Ketek supply and preamp box.

2. Switch on XIA Mercury.

3. Switch on Epics computer.

4. Go to a console on haspp10wg and type:

5. ssh -Y dxpconrad

Pw: generic... 
6. screen $-r R$

7. cd /home/epics/dxp-3-1/iocBoot/iocMercuryConrad/

8. ./START_IOC

9. if necessary, load fluo-macro in spec: qdo /usr/local/lib/spec.d/macros/xiadxp.mac

10. in spec, do: mcaon

\section{D.2.4. Energy Calibration}

Calibrate energy calculation via parameters a, b, c: edit/usr/local/lib/spec.d/macros/xiadxp.mac and search for

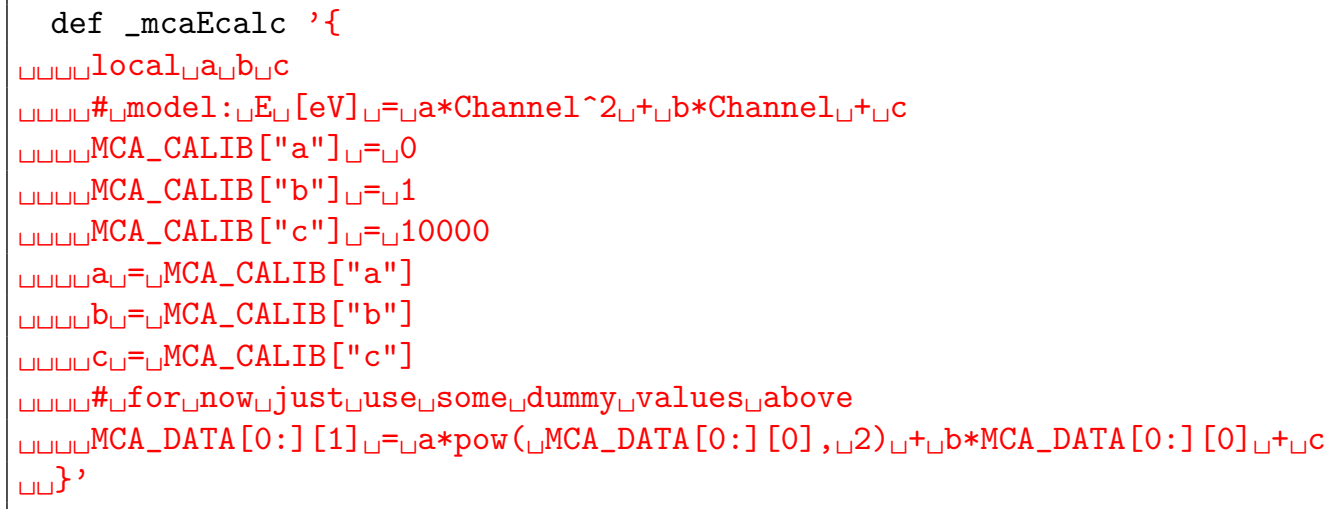

You may change the numbers behind MCA $\_$CALIB ["a"] =

Please note, that the calibration parameters of PyMCA are defined exactly in the opposite order, i. e., $\mathrm{a}=\mathrm{c}$ and v.v.

\section{D.2.5. Spec Commands}

mcaon activates mca from next scan/ct on mcaoff deactivates mca from next scan/ct on mcasetroi defines energy rois calculated for every acquisition (mcaROI0 mcaROI2)

usage mcasetroi counter_mne low_channel high_channel

e. g., mcasetroi mcaROI1 10100

_mca_clean_up a chance to get mca working again, when it is bricked

mcaploton activates plotting of spectra during measurement

mcaplotoff deactivates plotting of spectra during measurement

\section{D.2.6. Implementation into Spec}

Motor and Counter Device Configuration (Not CAMAC) SCALERS YES | dxpMercuryConrad: | 3 | EPICS PV as Counter

MCA- and CCD-Acquisition Type Device Configuration MCA-like 1 | YES | <Conrad:mca1 | EPICS MCA 
Scaler (Counter) Configuration Number Name Mnemonic $<>$ Device Unit Chan $<>$ Use As Scale Factor

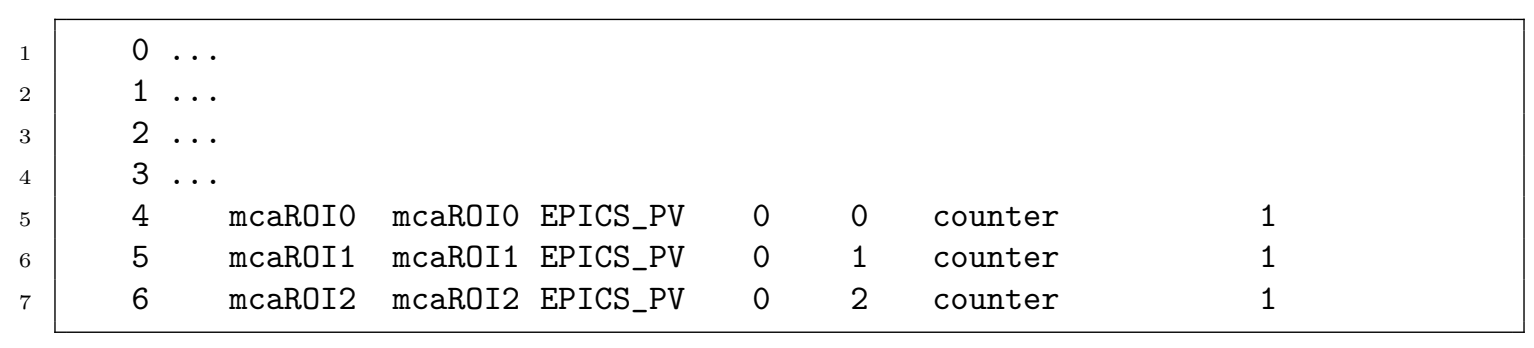

\section{D.2.7. Use Spec Counting Time for Mca}

The following commands are set in _mca_init of the macro xiadxp.mac

mca_par("soft_preset",1) If soft_preset is set to 0 the detector will not be programmed with spec's count time. If set to 1 the detector will be programmed with spec's count time.

mca_par("real") Spec will send the count time as real time. If this option is used in EPICS, "Mode" has to be set to Real Time. (default in xiadxp.mac)

mca_par("live") Spec will send the count time as live time (dead time corrected counting time). If this option is used in EPICS, "Mode" has to be set to Live Time. 


\section{List of Publications}

$12 / 2014$

$3 / 2014$

$2 / 2014$

$8 / 2012$

$1 / 2012$

2010
M. Priebe, M. Bernhardt, C. Blum, M. Tarantola, E. BoDenschatz, AND T. SAlditT: Scanning X-Ray Nanodiffraction on Dictyostelium discoideum. Biophysical Journal , 107(11):2662-2673.

T. Gorniak, T. Haraszti, V. M. Garamus, A. R. Buck, T. Senkbeil, M. Priebe, A. Hedberg-Buenz, D. Koehn, T. Salditt, M. Grunze, M. G. Anderson, and A. Rosenhahn: Nano-Scale Morphology of Melanosomes Revealed by Small-Angle X-Ray Scattering. PLoS ONE 9:e90884.

B. Weinhausen, O. Saldanha, R. N. Wilke, C. Dammann, M. Priebe, M. Burghammer, M. Sprung, and S. Köster: Scanning X-Ray Nanodiffraction on Living Eukaryotic Cells in Microfluidic Environments. Phys. Rev. Lett., 112(8):088102.

R. N. Wilke, M. Priebe, M. Bartels, K. Giewekemeyer, A. Diaz, P. KARVinen, AND T. SALDitT: Hard X-ray imaging of bacterial cells: nano-diffraction and ptychographic reconstruction. Opt. Express, 20(17):19232-19254.

M. Bartels, M. Priebe, R. N. Wilke, S. P. Krüger, K. Giewekemeyer, S. Kalbfleisch, C. Olendrowitz, M. Sprung, and T. SALDITT: Low-dose three-dimensional hard x-ray imaging of bacterial cells. Opt. Nanoscopy, 1:10.

M. Priebe, S. Kalbfleisch, M. Tolkiehn, S. Köster, B. Abel, R. J. DAVIES, AND T. SAlditT: Orientation of biomolecular assemblies in a microfluidic jet. New Journal of Physics, 12:043056. 